WSRC-TR-2003-00175

Publication Date: June 2003

\title{
Annual Radioactive Waste Tank Inspection Program - 2002 ${ }^{(\mathrm{u})}$
}

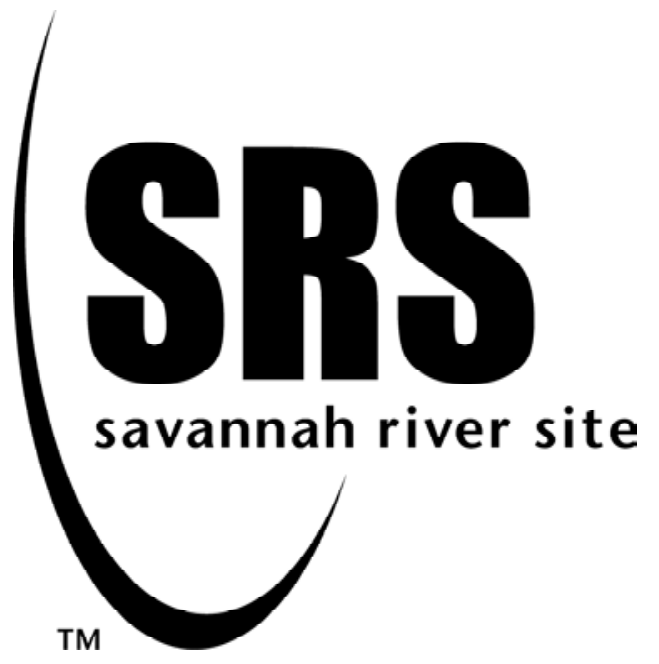

Westinghouse Savannah River Company

Savannah River Site

Aiken, SC 29808

Prepared for the U.S. Department of Energy under Contract No. DE-AC09-96SR18500 
This document was prepared in conjunction with work accomplished under Contract No. DE-AC09-96SR18500 with the U. S. Department of Energy.

\section{DISCLAIMER}

This report was prepared as an account of work sponsored by an agency of the United States Government. Neither the United States Government nor any agency thereof, nor any of their employees, makes any warranty, express or implied, or assumes any legal liability or responsibility for the accuracy, completeness, or usefulness of any information, apparatus, product or process disclosed, or represents that its use would not infringe privately owned rights. Reference herein to any specific commercial product, process or service by trade name, trademark, manufacturer, or otherwise does not necessarily constitute or imply its endorsement, recommendation, or favoring by the United States Government or any agency thereof. The views and opinions of authors expressed herein do not necessarily state or reflect those of the United States Government or any agency thereof.

This report has been reproduced directly from the best available copy.

Available for sale to the public, in paper, from: U.S. Department of Commerce, National Technical Information Service, 5285 Port Royal Road, Springfield, VA 22161, phone: (800) 553-6847, fax: (703) 605-6900

email: orders@ntis.fedworld.gov

online ordering: http://www.ntis.gov/help/index.asp

Available electronically at http://www.osti.gov/bridge

Available for a processing fee to U.S. Department of Energy and its contractors, in paper, from: U.S. Department of Energy, Office of Scientific and Technical Information, P.O. Box 62, Oak Ridge, TN 37831-0062,

phone: (865)576-8401,

fax: (865)576-5728

email: $\underline{\text { reports@ adonis.osti.gov }}$ 


\section{Annual Radioactive Waste Tank Inspection Program - $2002^{(\mathrm{U})}$}

R. S. Waltz, Jr.

W. R. West

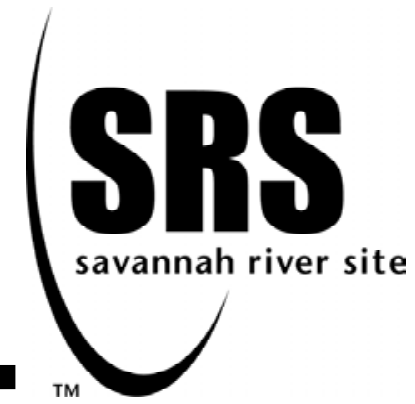


This page intentionally left blank. 


\section{Contents}

Acronyms and Abbreviations ....................................... 1

Introduction ..................................................................... 3

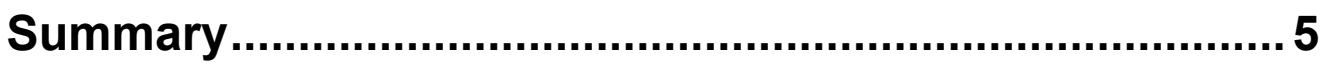

Inspection Program .................................................... 7

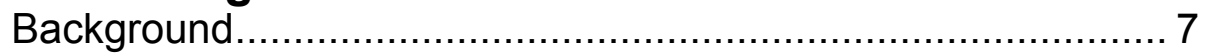

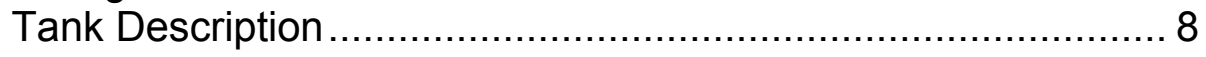

Inspection Methods ....................................................... 13

Program Implementation .............................................. 15

Visual Imagery .......................................................... 15

2002 Inspection Results ................................................... 15

Summary of Inspection Results ....................................... 16

Appendix A-Waste Tanks at SRS...................................23

Appendix B-Summary of 2002 Inspections .................... 25

\section{List of Figures}

1. Type I Waste Storage Tank............................................ 8

2. Type II Waste Storage Tank .......................................... 9

3. Type IV Waste Storage Tank ......................................... 10

4. Type III Waste Storage Tank .......................................... 11 
This page intentionally left blank. 
WSRC-TR-2003-00175

Annual Waste Tank

\section{Acronyms and Abbreviations}

\begin{tabular}{|c|c|}
\hline A & Annulus \\
\hline ASME & American Society of Mechanical Engineers \\
\hline BFV & Back Flush Valve \\
\hline CCTV & Closed Circuit Television \\
\hline CCWS & Chromate Cooling Water System \\
\hline COP & Clean Out Port \\
\hline CTS & Concentrate Transfer System \\
\hline CSTE & Concentration Storage and Transfer Engineering \\
\hline CWT & Concentrated Waste Tank \\
\hline DB & Diversion Box \\
\hline DOE-SR & Department of Energy-Savannah River \\
\hline DP & Direct Photography \\
\hline DWPF & Defense Waste Processing Facility \\
\hline ERIP & Encasement Riser Inspection Port \\
\hline ETF & Effluent Treatment Facility \\
\hline EVAP & Evaporator \\
\hline $\mathrm{F}$ & Fahrenheit \\
\hline GDL & Gravity Drain Line \\
\hline HELIUM & Helium leak test \\
\hline HLLCP & High Liquid Level Conductivity Probe \\
\hline HPFP & High Point Flush Pit \\
\hline I & Interior \\
\hline IAL & Intra-Area Line \\
\hline ISI & In-Service Inspection \\
\hline JB & Junction Box \\
\hline LDB & Leak Detection Box \\
\hline LPPP & Low Point Pump Pit \\
\hline LPS & Leak Probe Sleeve \\
\hline MLDB & Modified Leak Detection Box \\
\hline OD & Outside Diameter \\
\hline РHOTO & Photographs by Non-Remote Technique \\
\hline PP & Pump Pit \\
\hline psig & pounds per square inch gauge \\
\hline PSP & Periscopic Photography \\
\hline PT & Pump Tank \\
\hline $\mathrm{RCP}$ & Reinforced Concrete Pipe \\
\hline RTD & Resistance Thermo Device \\
\hline SRS & Savannah River Site \\
\hline SSD & Storm Sewer Drain \\
\hline SSMH & Storm Sewer Manhole \\
\hline STE & Shift Technical Engineer \\
\hline SWS & Storm Water Sewer \\
\hline TTJ & Telescopic Transfer Jet \\
\hline TTP & Telescopic Transfer Pump \\
\hline UT & Ultrasonic Test \\
\hline VB & Valve Box \\
\hline VP & Video Photograph \\
\hline WAP & Wide-Angle Photography \\
\hline WLE & Waste Line Encasement \\
\hline WSRC & Westinghouse Savannah River Company \\
\hline WT & Waste Transfer Line \\
\hline
\end{tabular}


This page intentionally left blank. 
WSRC-TR-2003-00175

Annual Waste Tank

Inspection Program - 2002

Introduction

\section{Introduction}

Aqueous radioactive wastes from Savannah River Site (SRS) separations and vitrification processes are contained in large underground carbon steel tanks. Inspections made during 2002 to evaluate these vessels and other waste

handling facilities along with evaluations based on data from previous inspections are the subject of this report. 
This page intentionally left blank. 
WSRC-TR-2003-00175

Annual Waste Tank

Inspection Program - 2002

\section{Summary}

The 2002 inspection program revealed that the structural integrity and waste confinement capability of the Savannah River Site waste tanks remained unchanged from 2001 with one exception.

During Ultrasonic Thickness (UT) mapping of Tank 15 a new through-wall crack was detected 129 inches above the tank bottom and three partial through-wall cracks were detected perpendicular to a vertical weld.

A total of 5006 photographs were made, 1006 visual and video inspections were performed, and 1 helium leak test was conducted. 
This page intentionally left blank. 


\section{Inspection Program}

\section{Background}

Alkaline aqueous radioactive wastes produced at the Savannah River Site are received and managed in large underground tanks. The waste came primarily from nuclear fuel reprocessing operations in the separations areas $(\mathrm{F}$ and $\mathrm{H})$ and contains most of the radioactive fission products from SRS operations. In addition, $\mathrm{H}$ tank farm receives recycle waste from the DWPF vitrification process. Some of this waste has been transferred to F Area waste tanks. The waste stored in the tanks is present in three phases: sludge, supernate, and salt formed by supernate evaporation and cooling. The supernate and salt phases consist primarily of $\mathrm{NaNO}_{3}$ and $\mathrm{NaNO}_{2}$. The fission product content is 1 to 20 curies per gallon for the supernate and 1 to 5 curies per gallon for the salt. The sludge consists primarily of $\mathrm{MnO}_{2}$ and $\mathrm{Fe}(\mathrm{OH})_{2}$ with a fission product content up to 950 curies per gallon.

Waste tank leak detection capabilities are essential to meet the primary objective of the SRS radioactive waste management program to manage the waste in such a manner as to minimize the radiation exposure and associated risk to man and his environment over the lifetime of the radionuclides.

The detection of leaked waste is based on two principles: disappearance of material from its proper location and appearance of material in an improper location. At SRS, primary reliance is on the latter because the quantity of the waste detectable in an improper location is much less than that detectable by inventory change in a large tank. Capacity of SRS tanks is 0.75 to 1.3 million gallons. Although rigorous tank inventory surveillance is practiced, primary leak detection methods rely on automatic surveillance of those areas into which the leaked waste is most likely to migrate.

The annulus of each double-wall tank is equipped with at least two single-point conductivity probes for leak detection. These probes are located at the bottom of the annulus and on opposite sides of the tank where possible. The single-wall tanks are built on slabs with a network of leak collection channels that drain to a common sump. Continuous sump level monitoring and frequent sump liquid sampling provide the leak detection. Besides the automatic surveillance, routine direct visual surveys are made in the annular spaces, and nonroutine direct visual surveys are made in primary tanks through opened access risers and/or inspection ports in the roof.

In 1961-62, following leakage of waste into the annuli of Tanks 9, 10, 14, and 16, the first remote imaging inspections were made of some tanks using a periscope. Random inspections continued through 1970. A program was initiated in November 1971 to periodically inspect all waste tanks, using remote visual imagery techniques to monitor for corrosion and other degradation, waste leakage, anomalies of any type, and to investigate process or equipment concerns.

Steel thickness measurements have been made periodically of waste tanks using ultrasonic techniques to monitor for general corrosion. An analog-type instrument was used in 1967 and 1969 to measure the thickness of the primary wall of selected double-wall tanks. In 1972, a more precise instrument was put in service. About 24,000 measurements made over a period of 14 years (1972 through 1985) indicated that no general thinning of SRS tanks had occurred. Steel thickness measurements were resumed in 1994 using an updated ultrasonic testing (UT) system.

To date, the only visually observed service-induced corrosion was in Tank 23, a tank with a unique service history. The upper wall interior surfaces show general corrosion with mild pitting. The pitting is broad but shallow. This tank was used to receive contaminated water from 244-H, the Receiving Basin for Off-Site Fuels, and 245-H, the Resin Regeneration Facility.

Inspections of waste tanks are complicated by factors such as radiation and radioactive contamination, remote operation as far as 40 feet below grade, and insertion of equipment through small (generally 5 to 8-inch-diameter) access openings. Inspection techniques to circumvent these difficulties have been developed, and they yield quality visual images and thickness measurements. The techniques include photographic systems, closed circuit television 
WSRC-TR-2003-00175

Annual Waste Tank

systems, and ultrasonic systems to measure steel thickness, cracking, and pitting.

Waste tank inspection has been important in leak detection. The leaksites in ten of thirteen cracked tanks have been identified by direct visual inspection or by one of the remote inspection techniques. Since the inspection program was initiated in 1971, eight tanks were found to have leaksites that were not recognized before the program was implemented. With one exception, Tank 6 in 2001, the annulus conductivity probes in the double-wall tanks were not activated by these leaks because of the small amount of leakage. The leaked waste evaporated to dryness, sealing the cracks before any leaked waste reached a leak detection probe. However, remote inspections detected the dry deposits of leaked waste in the annuli of these tanks.

The waste tank ISI program is an ongoing program. This report gives results of the 2002 inspections and summarizes significant findings of previous inservice inspections for each waste tank.

\section{Tank Description}

SRS has subsurface storage tanks of four different designs. All of the tanks are constructed of carbon steel and reinforced concrete. They serve as containment vessels for storage and processing of radioactive wastes. Appendix A lists tank location, design type, project number, and construction period. A brief description of the different tank designs is given in the following paragraphs.

\section{Type I Tanks}

The 12 original storage tanks constructed between 1951 and 1953 are designated Type I tanks. Tanks 1 through 8 are in F Area and Tanks 9 through 12 are in $\mathrm{H}$ Area. Each primary tank has a capacity of 750,000 gallons, and is 75 feet in diameter and $241 / 2$ feet high. Figure 1 shows the essential features of Type I tanks, including the primary tank, the secondary pan, and the concrete support structure.

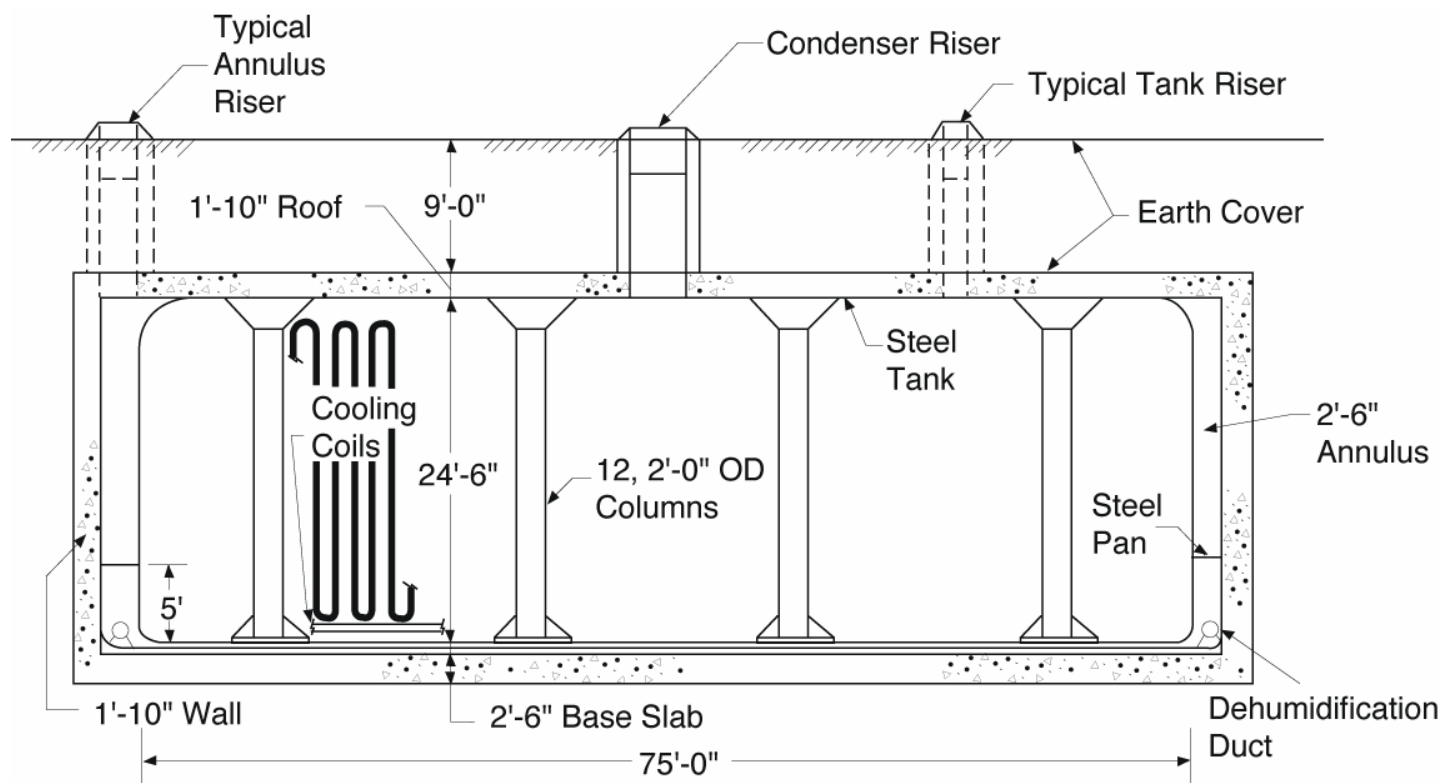

Figure 1. Cooled Waste Storage Tank, Type I (Original 750,000 Gallons) 
The primary container is a closed cylindrical tank with flat top and bottom constructed from 1/2-inchthick steel plates. The top and bottom are joined to the cylindrical sidewall by curved knuckle plates. The primary tank is set within a circular pan of 1/2inch-thick steel plates. The annulus pan is 5 feet deep and 5 feet larger in diameter than the primary tank, thus forming an annular space $21 / 2$ feet wide. The tank and pan are set on a 30-inch-thick base slab and are enclosed by a cylindrical 22-inch-thick reinforced concrete wall and a flat concrete roof, also 22 inches thick. There are twelve 2-foot-diameter concrete columns within the primary tank to support the roof. Each column has a flared capital and is encased in 1/2-inch-thick steel plate.

A 9-foot layer of earth was placed over the tanks for radiation shielding. Cooling for each Type I tank is provided by 36 parallel (water pipe) cooling coils.

A dehumidification duct in the annulus of each tank is routed from the tank top to the bottom of the annulus where it encircles all but 8 feet of tank. The duct has distribution outlets and its cross-sectional area decreases as the distance from the air supply increases. Access to the tank interior is provided at eight locations, and to the annular space at four locations, through riser pipes. Each of the 12 riser pipes is capped at the top with a concrete plug. Each plug is provided with two 5-inch-diameter ports equipped with removable plugs unless equipment has been installed to support waste removal or tank closure activities. The center plug port may provide access through three 4 to 8 inch diameter ports. Some of these ports provide access for inspections.

All welds in the pan and primary tank were radiographically inspected, defects were corrected, and the welds were rechecked radiographically. The welds in the flat bottoms of both the pan and the tank were vacuum-tested for leaks. Additionally, both vessels were hydrostatically tested. The water was maintained at full height in the tank for 24 hours before inspection for leaks was made. Cooling water piping was hydrostatically tested at 300 psig and then leak-tested with 100 psig air pressure in the piping.

\section{Type II Tanks}

Tanks 13 through 16, constructed in H Area in 1955 and 1956, are designated Type II tanks. Figure 2 is a cross section of this type. Each primary tank has a capacity of 1,030,000 gallons and is 85 feet in diameter and 27 feet high.

The primary container for Type II tanks consists of two concentric steel cylinders assembled with a flat bottom and a flat top into a form somewhat like a doughnut. The top and bottom are joined to the outer cylinder by rings of curved knuckle plates. The inner cylinder is flared at the top to accommodate the roof support column. This cylinder is joined to the flat steel top with a continuous butt weld and to a base fastened to the bottom with a continuous T-weld. Steel thicknesses are:

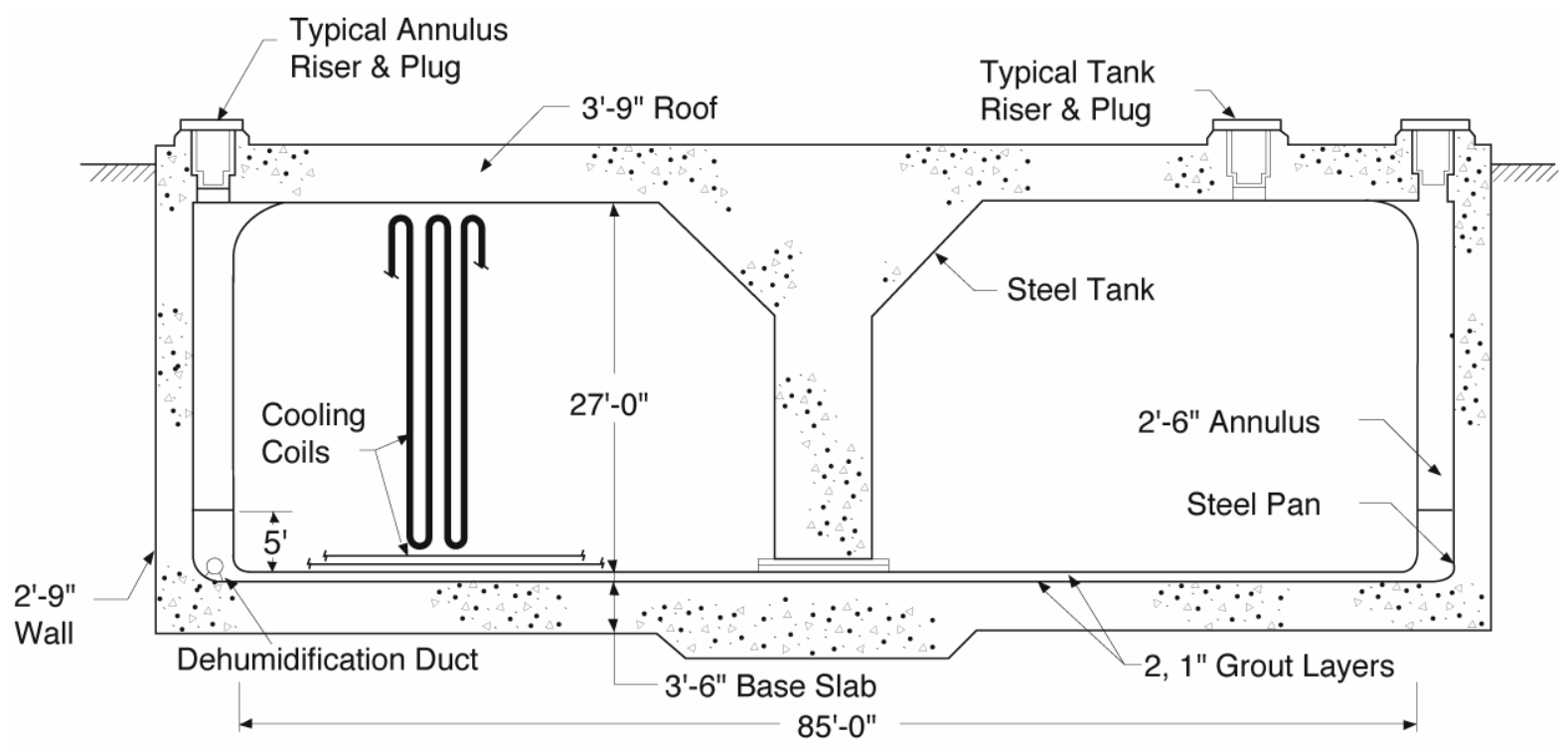

Figure 2. Cooled Waste Storage Tank, Type II (Original 1,030,000 Gallons) 
WSRC-TR-2003-00175

Annual Waste Tank

Plate

Top and bottom

Upper knuckle

Wall

Thickness, inch

Lower knuckle

$1 / 2$

$9 / 16$

$5 / 8$

$7 / 8$

The primary tank is set on a 1 -inch sand bed within a circular pan of 1/2-inch thick steel plate, 5 feet deep and 5 feet larger in diameter than the primary tank, thus forming an annular space $21 / 2$ feet wide. The tank and pan assembly is surrounded by a cylindrical reinforced concrete enclosure with a 33-inch-thick wall and a flat concrete roof that is 45 inches thick. The tank and pan assembly and the surrounding wall are set on a foundation slab that is 42 inches thick. The roof is supported by both the wall and a central concrete column that fits within the inner cylinder of the vessel. The 45 -inch-thick concrete roof provides radiation shielding; therefore, no earth overburden is required. Cooling for each Type II tank is provided by 44 parallel (water pipe) cooling coils. Access to the tank interior is provided at eight locations, and to the annular space at four locations, through riser pipes. Each of the 12 riser pipes is capped at the top with a concrete plug. Each plug is provided with two 5-inch-diameter ports equipped with removable plugs. The ports provide access for inspection. In addition to the four annulus risers, other access openings (10 to 14 additional openings per tank) have been drilled into the annulus of each of these tanks to permit inspection of seventy-three to ninety-six percent of the exterior walls of the primary vessels.
A dehumidification duct in the annulus of each tank is routed from the tank top to the bottom of the annulus where it encircles all but 8 feet of the tank. The duct has distribution outlets, and its crosssectional area decreases as the distance from the air supply increases.

All welds in the primary tanks were radiographically inspected, defects were corrected, and the welds were rechecked radiographically. However, the annulus pans were not inspected radiographically. The welds in the flat bottoms of these pans and the primary tanks were vacuum-tested for leaks, and the primary and secondary vessels were hydrostatically tested. Cooling water piping was hydrostatically tested at 300 psig and then leak-tested, with 100 psig air pressure in the piping.

\section{Type IV Tanks}

Tanks 17 through 24 are single-wall-uncooled tanks. These tanks were designed for storage of waste that does not require auxiliary cooling. Tanks 17 through 20 were constructed in F Area in 1958 and Tanks 21 through 24 were constructed in H Area between 1959 and 1961. Each tank has a capacity of $1,300,000$ gallons and is 85 feet in diameter and 34 feet high (Figure 3).

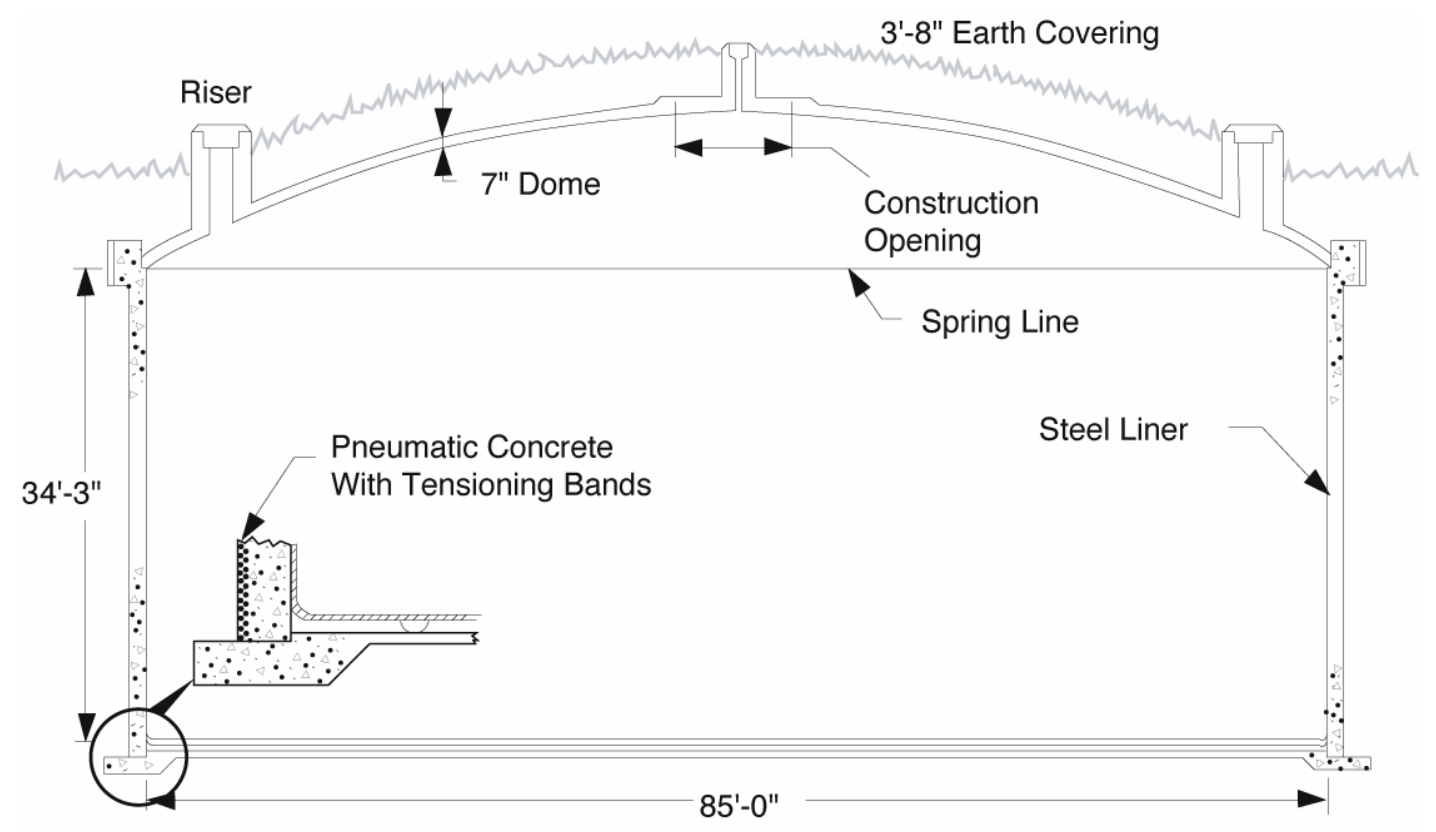

Figure 3. Uncooled Waste Storage Tank, Type IV (Prestressed Concrete Walls, 1,300,000 Gallons) 
WSRC-TR-2003-00175

Annual Waste Tank

Each Type IV tank is basically a steel-lined, prestressed-concrete tank in the form of a vertical cylinder with a domed roof. Carbon steel plates, $3 / 8$ inch thick, were used to form the cylindrical sides and flat bottom portion of the steel liners. The knuckle plates at the junction of the bottom and the sidewall are 7/16 inch thick. Concrete was built up around the steel vessel by the "shotcrete" technique.

Radiation shielding of the Type IV tanks in F Area was accomplished by applying at least 32 inches of earth over each of the 7-inch-thick concrete domes. $\mathrm{H}$-Area tanks were shielded similarly, except that the earth cover was at least 44 inches thick to accommodate a somewhat higher radiation level from the waste.

Access to the interior of the tank is provided at six locations through riser pipes. Each riser pipe is capped at the top with a concrete plug. Some of these risers provide access for inspection.

All welds in the steel liners were radiographically inspected. All of the welded tank-bottom seams and the upper seams of the knuckle rings were vacuum leak-tested. Prior to the back-filling operation, each tank was hydrostatically tested by filling with water to the normal fill line. The tank was allowed to remain filled until it was to be placed in use for waste storage.

\section{Type III Tanks}

The most recently constructed tanks are designated as Type III tanks (Figure 4). Twenty-seven tanks were built between 1967 and 1981. Tanks 25 through 28, 33 and 34, and 44 through 47 are located in F Area. Tanks 29 through 32, 35 through 43 and 48 through 51 are located in H Area.

The Type III tank design was developed after an investigation into the causes of the leaks from the primary vessel of the Type I and Type II tanks. The study concluded that the leak-producing mechanism was nitrate-induced, stress-corrosion cracking at sites in or near the weld seams, and that stress relieving after fabrication should eliminate the cracking. For the Type III tanks, means were provided for heating each finished tank to relieve the stresses generated during fabrication. In addition, some stress patterns were avoided, or minimized, by mounting the roof supporting column on the foundation pad rather than on the bottom of the primary tank (as in Types I and II), and by providing an annular clearance around the roof supporting column. Each primary tank holds $1,300,000$ gallons and is 85 feet in diameter and 33 feet high.

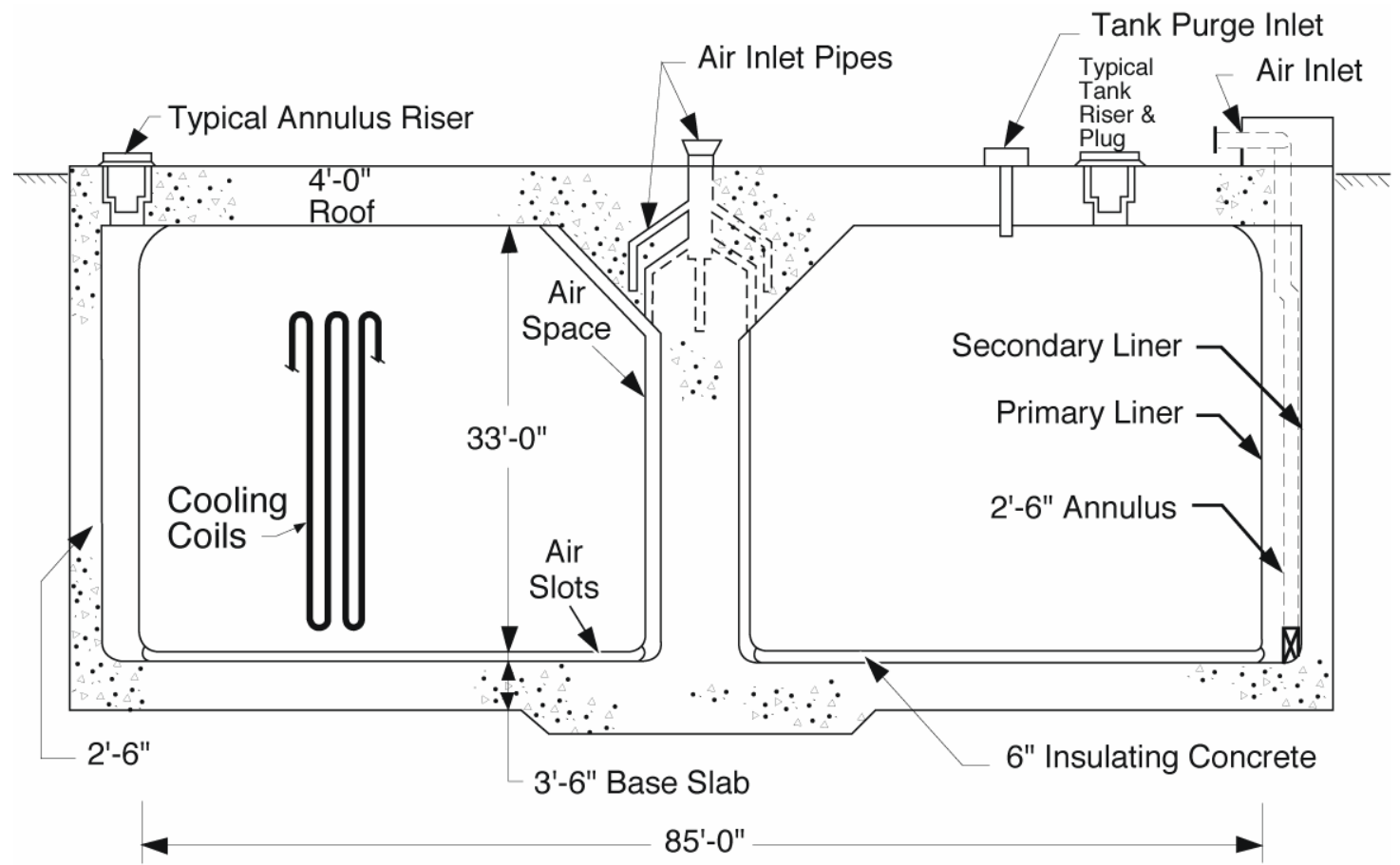

Figure 4. Cooled Waste Storage Tank, Type III (Stress Relieved Primary Liner, 1,300,000 Gallons) 
Type III tanks are similar to the doughnut-like design of Type II tanks. Each primary vessel is made of two concentric cylinders joined to washer-shaped top and bottom plates by curved knuckle plates. Steel thicknesses are:

Plate

Top and bottom

Upper knuckle

Outer wall

Upper band

Middle band

Lower band

Inner wall

Upper band

Lower band

Lower knuckle

Outer (tanks 25-28 and

33-51)

(tanks 29 through

32)

Inner

The primary tank is set on a 6-inch bed of insulating concrete within the secondary containment vessel. The concrete bed is grooved radially so that ventilating air can flow from the inner to the outer annulus. If any waste were to leak from the tank bottom or center annulus wall, liquid would move through the grooves, facilitating detection in the outer annulus.

The secondary vessel is 5 feet larger in diameter than the tank, thus providing an outer annulus $21 / 2$ feet wide. The secondary vessel is made of $3 / 8$-inch-thick steel throughout. Its sidewalls rise to the full height of the primary tank. The nested two-vessel assembly is surrounded by a cylindrical reinforced concrete enclosure with a 30-inch-thick wall. The enclosure has a 48-inch-thick flat reinforced concrete roof that is supported by the concrete wall and a central column that fits within the inner cylinder of the vessel. The 48 -inch-thick concrete provides radiation shielding; hence, no earth overburden is required.

Cooling for the Type III tanks is provided by either deployable (water pipe) cooling coil bundles installed through risers in the tank top, or 23 parallel (water pipe) cooling coils distributed throughout the tank.

A dehumidification duct in the annulus of each tank is routed from the tank top to the bottom of the annulus where it encircles the tank. The duct has distribution outlets and its cross-sectional area decreases as distance from the air supply increases. In these tanks, additional airflow is directed through the inner annulus, passing beneath the primary tank through radial grooves in the concrete base slab, and is exhausted into the outer annulus.

Tanks 29 through 34 were placed in service prior to 1976. These tanks were constructed with annulus riser pipes at four locations providing inspection access through 5-inch-diameter ports. All other Type III tanks were placed in service after 1976 and have annulus riser pipes at 18 locations that are 8 -inches in diameter. These ports are equidistant around the tank and provide for inspection of all of the exterior wall of the primary vessel. In 1982, fourteen to sixteen additional 8-inch diameter ports per tank were drilled in the tops of Tanks 29 through 34 to provide adequate access ports for inspection of all of the exterior wall of their primary vessels. All Type III tanks have interior riser pipes at various locations that provide inspection access through ports with diameters ranging from 4 to 8 inches. All inspection access ports are equipped with removable plugs.

All butt welds on the primary tanks were radiographically inspected, except welds on the horizontal roof surface. On the secondary vessels of Tanks 29 through 34, all butt welds joining bottom plates, knuckle plates, and the lowest courses of center-column and outer-wall plates, were radiographically inspected. On all other Type III tanks, all plate welds in the secondary tanks were radiographically inspected. All defects were corrected and the welds were rechecked radiographically.

The Quality Assurance Program included inspection of all radiographs by two independent groups of certified weld inspectors, and all radiographs were permanently stored for future reference. All spots on the inside or outside of the primary tanks and the inside of the secondary tanks, where clips or lugs were removed and where other excisions were made, were examined by magnetic particle or liquid penetrant techniques, and any defects were repaired.

All butt welds on the secondary tanks were vacuum leak-tested. All welds in the bottom assemblies of the primary tanks, including knuckle rings and lowest course welds, were vacuum leak-tested before each bottom assembly was lowered into final position, and then tested a second time after the stress-relieving operation. A full hydrostatic test, the filling of each primary tank to a depth of 32 feet and allowing it to stand 48 hours, was conducted after stress relieving. 
WSRC-TR-2003-00175

Annual Waste Tank

No leaks were found by the hydrostatic tests. All circumferential welds in the pipe loops of the deployable cooling coil bundles below the $1 / 2$-inchthick plate at the base of the riser plug were radiographed. The assembled cooler piping was tested hydrostatically to 500 psig and halide leaktested at 300 psig. Welds in the distributed cooling coils were radiographed and similarly leak-tested.

The primary tank was stress-relieved in place after all high temperature work (other than roof attachments) had been completed. Full stress relief, at $1100^{\circ} \mathrm{F}$, was accomplished in accordance with the general requirements of the ASME Boiler and Pressure Vessel code.

\section{Inspection Methods}

Techniques have been developed for remote examination and evaluation of the waste tanks and waste tank ancillaries. For visual imaging, direct photography systems developed at SRS were the primary method used. Closed circuit television systems were also used where direct photography was not possible or where these systems provided a more comprehensive examination. Only the direct photography systems will be described since the video systems are similar to systems used widely in industry.
Wide-angle direct photography was used for general inspections of double-wall tank annuli and the primary vessels of both double-wall tanks and singlewall tanks. This technique used a camera that surveys a large area in a single photograph. The camera used for wide-angle photography was a Contax G1 camera body, with a Zeiss Hologon $16 \mathrm{~mm}$ f/8 fixed aperture lens. This lens is distortion free with a field of view of approximately 100 degrees. A bank of four electronic flash units was synchronized with these cameras to provide illumination. This camera is not shielded since residence time in a radiation field is minimal.

Another direct photography technique was used for detailed inspections. The camera is shielded to reduce the degrading effect of ionizing radiation on the photographic film. The camera's residence time in a radiation field for this technique is longer than the wide-angle direct photographic technique (i.e., a few minutes versus a few seconds); hence, shielding is required. The camera used was the Contax G1 camera with a Zeiss Hologon $16 \mathrm{~mm} \mathrm{f} / 8$ lens, the same as used for the wide-angle direct photography. Illumination is provided by a single electronic flash unit. 
WSRC-TR-2003-00175

Annual Waste Tank

Inspection Program Inspection Program - 2002

This page intentionally left blank. 


\section{Program Implementation}

\section{Visual Imagery}

The 2002 inspection program used two visual imagery techniques: photography and closed circuit television. The primary inspection methods were direct photography techniques; e.g., making a series of photographs providing detailed views of the tank and wide-angle photography for obtaining overviews of large areas. Closed circuit television systems were generally used to further investigate conditions found during scheduled inspections and to document conditions and troubleshoot process problems in tanks and ancillaries.

The inspection program objective to continuously evaluate the waste tanks was satisfied in 2002 by photographic and videotape documentation. Inspections were made through all accessible annulus risers of the double-wall tanks and at least one inspection was made in the interior of each singlewall tank.

For Tanks 1 through 12, inspections are limited to no more than $25 \%$ of the exterior of the primary vessel wall and the annular space due to limited annulus access. These tanks are continuously monitored for leakage by instrumentation installed in their annuli. Additionally, for those tanks that have known leaksites in the primary vessel, the supernate phase has been removed, minimized, or the level lowered below the level of known leaksites.

\section{Inspection Results}

The 2002 inspection program was successfully completed. The annuli of all double-wall tanks were inspected via all accessible risers and the interiors of single-wall tanks remaining in service were inspected. Other inspections of waste tanks and ancillaries were performed as required by operating conditions and equipment performance.

Per the ISI plan, Tank 15 was selected for ultrasonic (UT) nondestructive examinations. Four 8.5 inch wide vertical strips for the entire accessible height, 30 feet $(10 \%)$ of horizontal weld, a vertical weld on the lower plate and five previously identified leak sites were examined for wall thinning, crack detection, flaw sizing, and pitting.

The UT examinations and findings are as follows:

IP-55 No reportable areas of thinning or pitting were detected in the 8.5 -inch vertical strip.

Baseline measurements were taken of a previously identified leaksite 200 inches above the tank bottom. The crack was measured on one side of a vertical weld on the upper plate. The crack was 3.7 inches long with 1.4 inches being completely through-wall.

IP-107 No reportable areas of thinning or pitting were detected in the 8.5 -inch vertical strip.

Baseline measurements were taken of a previously identified leaksite 88 inches above the tank bottom. The crack is located next to a weld attachment and could only be measured from one side.

The crack was measured to be 2.3 inches long through-wall on one side of the weld attachment (2.3-inch horizontal and 3.4-inch vertical).

IP-182 No reportable areas of thinning or pitting were detected in the 8.5 -inch vertical strip. A new partial through-wall crack indication was found 31 inches above the tank bottom. The crack is 0.55 inch long and $20 \%$ through-wall.

Three partial through-wall indications perpendicular to the vertical weld were detected at 49 inches above the tank bottom (60\% through-wall), 51 inches above the tank bottom (60\% through-wall), and 139 inches above the tank bottom (25\% throughwall).

One new through-wall crack detected was located 129 inches above the tank bottom and measured 4.5 inches long. This crack 
WSRC-TR-2003-00175

Annual Waste Tank

was not previously identified due to the absence of any salt deposits on the exterior of the primary tank.

Baseline measurements were taken of a previously identified leaksite at 172 feet, 150 inches above the tank bottom. The crack is located at a repair area in the middle girth weld and is 7.88 inches long with 7 inches being completely through-wall.

Baseline measurements were taken of a previously identified leaksite at 192 feet, 150 inches above the tank bottom. The crack is located at a repair area in the middle girth weld and is 18.1 inches long with 10.2 inches being completely through-wall.

IP-207 Baseline measurements were taken of a previously identified leaksite 150 inches above the tank bottom. The crack is located at a repair area in the middle girth weld and is 17.5 inches long with 12.7 inches being completely through-wall.

East(197) No reportable areas of thinning or pitting were detected in the 8.5 -inch vertical strip.

Rainwater continued to leak into the annulus of some tanks. Inleakage was evidenced primarily by surface stains, and occasionally by calciferous deposits, changed configuration in leaked waste in the annulus, and mild surface corrosion where annulus ventilation was inadequate to maintain a dry annulus.

Except as noted above, the conditions of the tanks remained essentially unchanged from the conditions reported in 2001. Details and results for inspections of the tanks and ancillaries performed in 2002 are listed in Appendix B.

\section{Summary of Inspection Results}

The following is a brief description of tank conditions as revealed by inspections and examinations made through 2002.

\section{Tank 1}

Tank 1 was placed in service in 1954. A small amount of dry waste was observed on the annulus floor in 1969. Subsequent inspections have revealed no additional leakage. Tank 1 was removed from active service in December 1985. Inspection of the exterior wall of the primary vessel is limited to $25 \%$ using existing inspection techniques through the four risers that provide access to the annulus. Visual examination of the observable portion of the tank wall has not revealed the location of the leak(s). Inspection photographs of the steel surface of the tank and the annulus have shown no significant surface corrosion or other anomalies. Ultrasonic measurements made in 1978, 1979, 1981, 1983, and 1985 showed that no detectable thinning of the tank wall had occurred.

\section{Tank 2}

Tank 2 was placed in service in 1955. Tank 2 was removed from active service in November 1984. Visual examinations of the observable portion (25\%) of the exterior of the primary vessel wall and the annulus have shown no leakage, significant surface corrosion, or other anomalies. Ultrasonic measurements made in 1967, 1972, 1973, 1977, 1981, and 1985 showed no detectable thinning of the tank wall.

\section{Tank 3}

Tank 3 was placed in service in 1956. Tank 3 was removed from active service in November 1984. Visual examinations of the observable portion $(25 \%)$ of the exterior of the primary vessel wall and the annulus have shown no leakage, significant surface corrosion, or other anomalies. Ultrasonic measurements made in 1973, 1977, 1981, and 1985 showed no detectable thinning of the tank wall.

\section{Tank 4}

Tank 4 was placed in service in 1961. Tank 4 was removed from active service in December 1979. Visual examinations of the observable portion $(25 \%)$ of the exterior of the primary vessel wall and the annulus have shown no leakage, significant surface corrosion, or other anomalies. Ultrasonic measurements made in 1973, 1977, 1981, and 1985 showed no detectable thinning of the tank wall.

\section{Tank 5}

Tank 5 was placed in service in 1959. Tank 5 was removed from active service in November 1990. Visual examinations of the observable portions $(25 \%)$ of the exterior of the primary vessel wall and the annulus through calendar year 2000 had shown no leakage, significant surface corrosion, or other anomalies. The tank was returned to active service in 2000 to support tank closure activities. Several 
WSRC-TR-2003-00175

Annual Waste Tank

Inspection Program - 2002

Program Implementation

months after liquid was added to the tank, a magnetically mounted wall crawler with a video camera attached was deployed which enabled $75 \%$ of the primary vessel wall to be inspected. These inspections revealed 15 leaksites. Less than 5 gallons of waste reached the annulus floor. Ultrasonic measurements made in 1973, 1977, 1981, and 1985 showed no detectable thinning of the tank wall.

\section{Tank 6}

Tank 6 was placed in service in 1964. Tank 6 was removed from active service in October 1990. Visual examinations of the observable portions $(25 \%)$ of the exterior of the primary vessel wall and the annulus through calendar year 2000 had shown no leakage, significant surface corrosion, or other anomalies. The tank was returned to active service in 2000 to support tank closure activities. The first indication of leakage from the tank was in January 2001 when an annulus conductivity probe alarm was received. Liquid was observed on the annulus floor; however, no leaksites could be located from the four risers used to inspect the tank. In February, a magnetically mounted wall crawler with a video camera attached enabled $73 \%$ of the primary vessel wall to be examined. These inspections identified 6 leaksites. Approximately 92 gallons of waste reached the annulus floor. Ultrasonic measurements made in 1974, 1977, 1978, 1979, 1981, and 1985 showed no detectable thinning of the tank wall.

\section{Tank 7}

Tank 7 was placed in service in 1954. Tank 7 was removed from active service in November 1989. Visual examinations of the observable portion (25\%) of the exterior of the primary vessel wall and the annulus have shown no leakage, significant surface corrosion, or other anomalies. Ultrasonic measurements made in 1974, 1979, 1981, 1983, and 1985 showed no detectable thinning of the tank wall. Tank 7 was returned to service in 2002 to support waste removal activities.

\section{Tank 8}

Tank 8 was placed in service in 1956. Tank 8 was removed from active service in September 1992. Waste removal activities were conducted during 2001. A magnetically mounted wall crawler with a video camera attached enabled inspection of $59 \%$ of the primary vessel wall and annulus. No leakage, significant surface corrosion, or other anomalies were observed. Ultrasonic measurements made in 1973, 1977, 1981, and 1985 showed no detectable thinning of the tank wall.

\section{Tank 9}

Tank 9 was placed in service in 1955. Tank 9 was removed from active service in July 1991. Leakage from the tank primary vessel into the annulus pan may have occurred as early as 1955 when the "necklace" alarm, a conductivity leak detection device, shorted out permanently. Leakage was not certain until liquid waste was observed in the annulus pan in 1957. Currently, the annulus pan contains 8 to 10 inches of dry leaked waste. Visual examinations of the observable portion (13\%) of the exterior of the primary vessel wall have shown three leaksites high on the tank wall; 269, 271, and 276 inches above the tank bottom. None of these leaksites is the source of the leaked waste in the annulus pan. The waste leaked at these sites was only enough to form localized small nodules. The leak(s) that are the source of the waste in the annulus pan have not been observed. Inspections have shown no significant surface corrosion, and the ultrasonic measurements made in 1979 and 1983 showed no detectable thinning of the tank wall.

\section{Tank 10}

Tank 10 was placed in service in 1955 . Tank 10 was removed from active service in August 1989. The first indication that Tank 10 had leaked was in 1959 when dry waste was discovered in the annulus pan during a visual inspection. Currently, the annulus pan contains about 2 inches of dry leaked waste. Visual examinations of the observable portion $(19 \%)$ of the exterior of the primary vessel wall have not shown the source of the leaked waste or any other leaksite(s). Inspections have shown no significant surface corrosion, and the ultrasonic measurements made in 1979 and 1983 showed no detectable thinning of the tank wall.

\section{Tank 11}

Tank 11 was placed in service in 1955 . Tank 11 was removed from active service in July 1989. Twentyfive percent of the exterior of the primary vessel wall is observable via the four risers that provide access to the annulus. Inspections performed in 1974 revealed two leaksites. The leaksites are 189 and 235 inches above the tank bottom. Inspections have shown no significant surface corrosion, and ultrasonic measurements made in 1973, 1977, 1981, and 1985 showed no detectable thinning of the tank wall. 
WSRC-TR-2003-00175

Annual Waste Tank

\section{Tank 12}

Tank 12 was placed in service in 1956 . Tank 12 was removed from active service in July 1990. Twentyfive percent of the exterior of the primary vessel wall is observable via the four risers that provide access to the annulus. Inspections in 1974 revealed two leaksites. The leaksites are 93 and 105 inches above the tank bottom. Inspections have shown no significant surface corrosion, and ultrasonic measurements made in 1972, 1973, 1977, 1981, 1983, and 1985 showed no detectable thinning of the tank wall.

\section{Tank 13}

Tank 13 was placed in service in 1956. Tank 13 was removed from active service in May 1991. Ninety percent of the exterior of the primary vessel wall is observable via the 13 risers that provide access to the annulus. Inspections in 1977 revealed a leaksite 279 inches above the tank bottom. In 1980, another leaksite was discovered 269 inches above the tank bottom. Inspections have shown no significant surface corrosion, and ultrasonic measurements made in 1974, 1979, 1985 and 2002 showed no detectable thinning of the tank wall.

\section{Tank 14}

Tank 14 was placed in service in 1957. Tank 14 was removed from active service in December 1991. The first indication that tank 14 had leaked was in 1959 when dry leaked waste was observed in the annulus pan. Currently, the annulus pan contains 12 to 13 inches of dry leaked waste. Eighty-nine percent of the exterior of the primary vessel wall is observable via the 18 risers that provide access to the annulus. Inspections have located 33 leaksites, and it is estimated that there are about 50 leaksites in this tank. All of the observed leaksites are near the bottom circumferential weld that is 2.5 feet above the tank bottom, except for one leaksite that was observed approximately 24 feet above the tank bottom. Inspections have shown no significant surface corrosion, and ultrasonic measurements made in 1979 and 1983 showed no detectable thinning of the tank wall.

\section{Tank 15}

Tank 15 was placed in service in 1960 . Tank 15 was removed from active service in November 1989. Inspections in 1972 below one of the four risers providing access to the annulus revealed two leaksites near the bottom circumferential weld about
2.5 feet above the tank bottom. Twelve additional risers were installed, increasing the observable portion of the primary vessel wall from $25 \%$ to $96 \%$. Inspections in 1973, via the additional risers, revealed eleven other leaksites. Later, inspections revealed three other sites where cracks penetrated the steel wall, one was observed in 1994 and two were observed in 1997. Inspections in 2000 revealed two additional leaksites near the bottom circumferential weld. A total of 18 leaksites have been identified.

Inspections have shown mild corrosion of the steel surfaces in the tank annulus. Ultrasonic measurements made in 1972, 1977, 1980, 1984, and 2002 showed no reportable thinning of the tank wall. Using new equipment capable of detecting cracks and pitting, four partial through-wall crack indications and one complete through-wall crack were identified during UT mapping in 2002.

\section{Tank 16}

Tank 16 was placed in service in 1959. Tank 16 was removed from active service in February 1979. Liquid waste was detected in the annulus pan in 1959. Seventy-three percent of the exterior wall of the primary vessel is observable via the sixteen risers that provide access to the annulus. Inspections in 1961 and 1962, through 13 risers, revealed about 175 leaksites in the tank wall. In October 1961 and March 1962, two 5 3/4-inch-diameter samples were cut from the top horizontal circumferential weld of the tank wall about 40 feet apart. Metallurgical examination indicated that the cause of the cracks was nitrateinduced stress corrosion. Extensive inspection performed since 1972 indicated that the primary vessel wall has 300 to 350 leaksites. In $1978,70 \%$ of the leaked waste in the annulus pan was removed, leaving an insoluble heel containing approximately 30,000 curies Cs-137. Waste removal from the interior of the primary vessel was completed in 1980 , and the tank status changed to "out of service". Inspections have shown no significant surface corrosion. No ultrasonic steel thickness measurements of the tank were made because of the number of leaksites and the presence of leaked waste deposits on the primary vessel exterior.

\section{Tank 17}

Tank 17 was placed in service in 1961. Visual examinations of the steel liner have shown no evidence of failure, significant surface corrosion, or other anomalies. Tank 17 was removed from service and closed December 15, 1997. Inspections have been discontinued. 
WSRC-TR-2003-00175

Annual Waste Tank

Inspection Program - 2002

Program Implementation

\section{Tank 18}

Tank 18 was placed in service in 1959. Visual examinations of the steel liner have shown no evidence of failure, significant surface corrosion, or other anomalies. Ultrasonic measurements made in 1977, 1980, and 1983 showed no detectable thinning of the liner bottom. Activities to remove all waste from the tank began in 2001 .

\section{Tank 19}

Tank 19 was placed in service in 1961 and emptied in 1981. The tank has remained empty except for ballast water. Visual examinations of the steel liner have revealed two failures; i.e. sites where inleakage had occurred. The failures are in the wall of the steel liner at heights of 317 inches and 330 inches. Inspection records photographically document that these leaksites existed before 1994. However, inspections made from the interior of this single-wall (visual inspection of the exterior is not possible) had to track changes in artifacts at the sites by periodic observation to judge that inleakage had occurred. Ultrasonic measurements made in 1982 and 1985 showed no detectable thinning of the liner bottom. Activities to remove all waste from the tank began in 2000.

\section{Tank 20}

Tank 20 was placed in service in 1960. Visual examinations of the steel liner have revealed four failure sites. In 1983, leaksites were observed in the wall of the steel liner at heights of 22, 24.5, and 26.5 feet. In 1990, a leaksite was confirmed in the liner wall at a height of 26.25 feet. This site had been suspect since 1984 . This is a single-wall tank with no annulus. The leaksites in the steel liner were detected by inspections made from the tank interior, since inspection of the exterior was not possible. Artifacts observed on the interior wall indicated that water had leaked through the steel liner into the tank. It is possible that a small quantity of waste may have leaked from the steel liner. However, groundwater monitoring has given no indication that waste escaped the encasement. Tank 20 was removed from service and closed July 31, 1997. Inspections have been discontinued.

\section{Tank 21}

Tank 21 was placed in service in 1961. Visual examinations of the steel liner have shown no evidence of failure, significant surface corrosion, or other anomalies. Ultrasonic measurements made in
1973, 1977, 1980, and 1983 showed no detectable thinning of the liner bottom.

\section{Tank 22}

Tank 22 was placed in service in 1965. Visual examinations of the steel liner have shown no evidence of failure, significant surface corrosion, or other anomalies. Water was discovered leaking through the concrete roof in 1994. Ultrasonic measurements made in 1974, 1977, 1980, and 1983 showed no detectable thinning of the liner bottom.

\section{Tank 23}

Tank 23 was placed in service in 1964. Visual examinations of the steel liner have revealed corrosion but no evidence of failure. Ultrasonic measurements made in 1973, 1977, 1980, and 1983 showed no detectable thinning of the liner bottom. Examinations of the steel liner have shown rust and tubercles on the surface of the upper portion. This tank served as a receiver tank for inhibited contaminated water from Buildings $244-\mathrm{H}$, the Receiving Basin for Off-Site Fuels, and 245-H, the Resin Regeneration Facility. The tank was filled to less than $50 \%$ capacity to maintain the remaining space for emergency use. This mode of operation exposed only the lower half of the tank to the inhibited contents and exposed the upper half of the tank to a warm humid atmosphere. In 1984, rust and tubercles were cleaned from two small areas, exposing the steel surface. The cleaned liner surface was generally corroded with mild pitting. The pits were broad and shallow. In 1999, cracked or crushed concrete was noted in the tank dome, spanning about fifteen feet immediately above the tank wall.

\section{Tank 24}

Tank 24 was placed in service in 1963. Visual examinations of the steel liner have shown no evidence of failure, significant surface corrosion, or other anomalies. Ultrasonic measurements made in 1984 showed no detectable thinning of the liner.

\section{Tank 25}

Tank 25 was placed in service in 1980. Visual examinations of $100 \%$ of the exterior of the primary vessel wall and the annulus have shown no leakage, significant surface corrosion, or other anomalies. Ultrasonic thickness measurements made in 1979 and 1983 showed no detectable thinning of the tank wall. 


\section{Tank 26}

Tank 26 was placed in service in 1980. Visual examinations of $100 \%$ of the exterior of the primary vessel wall and the annulus have shown no leakage, significant surface corrosion, or other anomalies. Ultrasonic thickness measurements made in 1979 and 1983 showed no detectable thinning of the tank wall.

\section{Tank 27}

Tank 27 was placed in service in 1980. Visual examinations of $100 \%$ of the exterior of the primary vessel wall and the annulus have shown no leakage, significant surface corrosion, or other anomalies. Ultrasonic thickness measurements made in 1979 and 1983 showed no detectable thinning of the tank wall.

\section{Tank 28}

Tank 28 was placed in service in 1980. Visual examinations of $100 \%$ of the exterior of the primary vessel wall and the annulus have shown no leakage, significant surface corrosion, or other anomalies. Ultrasonic thickness measurements made in 1979 and 1983 showed no detectable thinning of the tank wall.

\section{Tank 29}

Tank 29 was placed in service in 1971. Visual examinations of $100 \%$ of the exterior of the primary vessel wall and the annulus have shown no leakage, significant surface corrosion, or other anomalies. Ultrasonic thickness measurements made in 1973 and 1974 showed no detectable thinning of the tank wall.

\section{Tank 30}

Tank 30 was placed in service in 1974. Visual examinations of $100 \%$ of the exterior of the primary vessel wall and the annulus have shown no leakage, significant surface corrosion, or other anomalies. Ultrasonic thickness measurements made in 1975 showed no detectable thinning of the tank wall.

\section{Tank 31}

Tank 31 was placed in service in 1972. Visual examinations of $100 \%$ of the exterior of the primary vessel wall and the annulus have shown no leakage, significant surface corrosion, or other anomalies.

\section{Tank 32}

Tank 32 was placed in service in 1971. Visual examinations of $100 \%$ of the exterior of the primary vessel wall and the annulus have shown no leakage, significant surface corrosion, or other anomalies.

\section{Tank 33}

Tank 33 was placed in service in 1969. Visual examinations of $100 \%$ of the exterior of the primary vessel wall and the annulus have shown no leakage, significant surface corrosion, or other anomalies.

\section{Tank 34}

Tank 34 was placed in service in 1972. Visual examinations of $100 \%$ of the exterior of the primary vessel wall and the annulus have shown no leakage, significant surface corrosion or other anomalies.

\section{Tank 35}

Tank 35 was placed in service in 1977. Visual examinations of $100 \%$ of the exterior of the primary vessel wall and the annulus have shown no leakage, significant surface corrosion, or other anomalies. Ultrasonic thickness measurements made in 1977, 1981, and 1985 showed no detectable thinning of the tank wall.

\section{Tank 36}

Tank 36 was placed in service in 1977. Visual examinations of $100 \%$ of the exterior of the primary vessel wall and the annulus have shown no leakage, significant surface corrosion, or other anomalies. Ultrasonic thickness measurements made in 1977, 1981, and 1985 showed no detectable thinning of the tank wall.

\section{Tank 37}

Tank 37 was placed in service in 1978. Visual examinations of $100 \%$ of the exterior of the primary vessel wall and the annulus have shown no leakage, significant surface corrosion, or other anomalies. Ultrasonic thickness measurements made in 1977, 1981, and 1985 showed no detectable thinning of the tank wall.

\section{Tank 38}

Tank 38 was placed in service in 1981. Visual examinations of $100 \%$ of the exterior of the primary vessel wall and the annulus have shown no leakage, significant surface corrosion, or other anomalies. Ultrasonic thickness measurements made in 1980, 1981, and 1984 showed no detectable thinning of the tank wall. 
WSRC-TR-2003-00175

Annual Waste Tank

Inspection Program - 2002

Program Implementation

\section{Tank 39}

Tank 39 was placed in service in 1982. Visual examinations of $100 \%$ of the exterior of the primary vessel wall and the annulus have shown no leakage, significant surface corrosion, or other anomalies. Ultrasonic thickness measurements made in 1980, 1981, 1984, and 1985 showed no detectable thinning of the tank wall.

\section{Tank 40}

Tank 40 was placed in service in 1986. Visual examinations of $100 \%$ of the exterior of the primary vessel wall and the annulus have shown no leakage, significant surface corrosion, or other anomalies. Ultrasonic thickness measurements were made in 1980, 1981, and 1984. Thickness mapping was performed in 1996 using the P-scan System to provide reference measurements for the future. No service-induced corrosion was detected.

\section{Tank 41}

Tank 41 was placed in service in 1982. Visual examinations of $100 \%$ of the exterior of the primary vessel wall and the annulus have shown no leakage, significant surface corrosion, or other anomalies. Ultrasonic thickness measurements made in 1980, 1981, and 1984 showed no detectable thinning of the tank wall.

\section{Tank 42}

Tank 42 was placed in service in 1982. Visual examinations of $100 \%$ of the exterior of the primary vessel wall and the annulus have shown no leakage, significant surface corrosion, or other anomalies. Ultrasonic thickness measurements were made in 1980, 1981, 1984, 1985, and 1990. Thickness mapping was performed in 1995 and 1996 using the P-scan System to provide reference measurements for the future. No service-induced corrosion was detected.

\section{Tank 43}

Tank 43 was placed in service in 1982. Visual examinations of $100 \%$ of the exterior of the primary vessel wall and the annulus have shown no leakage, significant surface corrosion, or other anomalies. Ultrasonic thickness measurements made in 1980, 1981, 1984, and 1985 showed no detectable thinning of the tank wall.

\section{Tank 44}

Tank 44 was placed in service in 1982. Visual examinations of $100 \%$ of the exterior of the primary vessel wall and the annulus have shown no leakage, significant surface corrosion, or other anomalies. Ultrasonic thickness measurements made in 1980, 1981, and 1984 showed no detectable thinning of the tank wall.

\section{Tank 45}

Tank 45 was placed in service in 1982. Visual examinations of $100 \%$ of the exterior of the primary vessel wall and the annulus have shown no leakage, significant surface corrosion, or other anomalies. Ultrasonic thickness measurements made in 1980, 1981, and 1984 showed no detectable thinning of the tank wall.

\section{Tank 46}

Tank 46 was placed in service as an emergency spare tank in 1980. It was placed in waste storage service in 1994 when it began receiving concentrate from the $2 \mathrm{~F}$ evaporator. Visual examinations of $100 \%$ of the exterior of the primary vessel wall and the annulus have shown no significant surface corrosion or other anomalies. Ultrasonic thickness measurements made in 1980, 1981, and 1984 showed no detectable thinning of the tank wall.

\section{Tank 47}

Tank 47 was placed in service in 1980. Visual examinations of $100 \%$ of the exterior of the primary vessel wall and the annulus have shown no leakage, significant surface corrosion, or other anomalies. Ultrasonic thickness measurements made in 1980, 1981, and 1984 showed no detectable thinning of the tank wall.

\section{Tank 48}

Tank 48 was placed in service in 1983. Visual examinations of $100 \%$ of the exterior of the primary vessel wall and the annulus have shown no leakage, significant surface corrosion, or other anomalies. Ultrasonic thickness measurements made in 1982. Thickness mapping was performed in 1994, 1995, 1996, and 1997 using the P-scan System to provide reference measurements for the future. No serviceinduced corrosion was detected. 
WSRC-TR-2003-00175

Annual Waste Tank

\section{Tank 49}

Tank 49 was placed in service in 1983. Visual examinations of $100 \%$ of the exterior of the primary vessel wall and the annulus have shown no leakage, significant surface corrosion, or other anomalies. Ultrasonic thickness measurements were made in 1982 prior to placing the tank in service. Thickness mapping was performed in 1995 using the P-scan System to provide reference measurements for the future. No service-induced corrosion was detected.

\section{Tank 50}

Tank 50 was placed in service in 1983. Visual examinations of $100 \%$ of the exterior of the primary vessel wall and the annulus have shown no leakage, significant surface corrosion, or other anomalies.
Ultrasonic thickness measurements were made in 1982. Thickness mapping was performed in 1994 and 1995 using the P-scan System to provide reference measurements for the future. No service-induced corrosion was detected.

\section{Tank 51}

Tank 51 was placed in service in 1986. Visual examinations of $100 \%$ of the exterior of the primary vessel wall and the annulus have shown no leakage, significant surface corrosion, or other anomalies. Ultrasonic thickness measurements were made in 1982. Thickness mapping was performed in 1996 and 1997 using the P-scan System to provide reference measurements for the future. No service-induced corrosion

detected. 
WSRC-TR-2003-00175

Annual Waste Tank

Inspection Program - 2002

Appendix A-Waste Tanks at SRS

\section{Appendix A-Waste Tanks at SRS}

SRS Waste Tank Specifications

\begin{tabular}{|c|c|c|c|c|c|}
\hline Number & Location & Type & $\begin{array}{l}\text { Project } \\
\text { Number } \\
\end{array}$ & $\begin{array}{c}\text { Construction } \\
\text { Period } \\
\end{array}$ & $\begin{array}{c}\text { Type of } \\
\text { Construction* }\end{array}$ \\
\hline $1-8$ & $\mathrm{~F}$ & I & 8980 & $1951-1953$ & Double wall-cooled \\
\hline $9-12$ & $\mathrm{H}$ & I & 8980 & $1951-1953$ & Double wall-cooled \\
\hline $13-16$ & $\mathrm{H}$ & II & $\begin{array}{c}8980 \\
\text { P.W.O. }\end{array}$ & $1955-1956$ & Double wall-cooled \\
\hline $17-20$ & $\mathrm{~F}$ & IV & 981031 & 1958 & Single wall-uncooled \\
\hline $21-24$ & $\mathrm{H}$ & IV & 981089 & 1962 & Single wall-uncooled \\
\hline $25-28$ & $\mathrm{~F}$ & IIIA & $\begin{array}{l}951493 \\
(75-1-a)\end{array}$ & $1975-1978$ & Double wall-cooled \\
\hline $29-32$ & $\mathrm{H}$ & III & 981232 & $1967-1970$ & Double wall-cooled \\
\hline $33-34$ & $\mathrm{~F}$ & III & 950974 & 1969-1972 & Double wall-cooled \\
\hline $35-37$ & $\mathrm{H}$ & IIIA & $\begin{array}{l}951463 \\
(74-1-a)\end{array}$ & 1974-1977 & Double wall-cooled \\
\hline $38-43$ & $\mathrm{H}$ & IIIA & $\begin{array}{l}951618 \\
(76-8-A)\end{array}$ & $1976-1980$ & Double wall-cooled \\
\hline $44-47$ & $\mathrm{~F}$ & IIIA & 951747 & $1977-1980$ & Double wall-cooled \\
\hline $48-51$ & $\mathrm{H}$ & IIIA & $\begin{array}{l}951828 \\
(78-18-b)\end{array}$ & $1978-1981$ & Double wall-cooled \\
\hline
\end{tabular}

* Tanks 32 and 35 have removable, roof-supported cooling coils. Tanks 30, 33, and 34 have bottom-supported deployable cooling coils. Tanks 29 and 31 have some deployable and some close-packed cooling assemblies, all bottom supported. All other cooled tanks have permanently installed cooling coils, roof-supported in Type I and II and bottom-supported in Type III tanks. 
WSRC-TR-2003-00175

Annual Waste Tank

Appendix A-Waste Tanks at SRS

Inspection Program - 2002

This page intentionally left blank. 


\section{Appendix B-Summary of 2002 Inspections}

\begin{tabular}{|c|c|c|c|c|c|c|c|c|}
\hline \multirow{2}{*}{$\frac{\text { AREA }}{\mathrm{F}}$} & \multirow{2}{*}{$\begin{array}{c}\begin{array}{c}\text { TANK OR } \\
\text { ANCILLARY }\end{array} \\
01\end{array}$} & \multicolumn{2}{|c|}{$\frac{\text { ACCESS OPENING }}{\text { (A OR I) }}$} & \multirow{2}{*}{$\frac{\text { DATE }}{06 / 11 / 02}$} & \multicolumn{3}{|c|}{$\begin{array}{l}\text { INSPECTION METHOD } \\
\text { IDENTIFICATION NUMBER }\end{array}$} & \multirow{2}{*}{$\frac{\text { REMARKS }}{\text { Tank condition had not changed. }}$} \\
\hline & & East & (A) & & DP & I & P02169:01-23 & \\
\hline $\mathrm{F}$ & 01 & East & (A) & 06/13/02 & CCTV & I & $808 \mathrm{C}$ & $\begin{array}{l}\text { The conductivity probe and } \\
\text { magnetically mounted } \\
\text { thermocouple were deployed } \\
\text { within the setpoint limits. }\end{array}$ \\
\hline $\mathrm{F}$ & 01 & North & (A) & $06 / 11 / 02$ & DP & I & P02168:01-23 & Tank condition had not changed. \\
\hline $\mathrm{F}$ & 01 & South & (A) & $06 / 11 / 02$ & DP & I & P02167:01-23 & Tank condition had not changed. \\
\hline $\mathrm{F}$ & 01 & West & (A) & 06/11/02 & DP & I & P02166:01-24 & Tank condition had not changed. \\
\hline $\mathrm{F}$ & 01 & West & (A) & $06 / 13 / 02$ & CCTV & l & $808 \mathrm{C}$ & $\begin{array}{l}\text { The conductivity probe was } \\
\text { deployed within the setpoint } \\
\text { limits. }\end{array}$ \\
\hline $\mathrm{F}$ & 02 & East & (A) & 03/06/02 & CCTV & / & $808 \mathrm{~B}$ & $\begin{array}{l}\text { The magnetically mounted } \\
\text { thermocouple was deployed } \\
\text { within the setpoint limits. }\end{array}$ \\
\hline $\mathrm{F}$ & 02 & East & (A) & 03/15/02 & WAP & I & P02147:03 & Tank condition was normal. \\
\hline $\mathrm{F}$ & 02 & North & (A) & 03/05/02 & DP & l & P02104:01-22 & $\begin{array}{l}\text { Tank condition was normal. A } \\
\text { masselin cloth was observed on } \\
\text { the ventilation duct. }\end{array}$ \\
\hline $\mathrm{F}$ & 02 & North & (A) & 03/06/02 & CCTV & l & $808 \mathrm{~B}$ & $\begin{array}{l}\text { The conductivity probe was } \\
\text { deployed within the setpoint } \\
\text { limits. }\end{array}$ \\
\hline $\mathrm{F}$ & 02 & South & (A) & 03/06/02 & CCTV & l & $808 \mathrm{~B}$ & $\begin{array}{l}\text { The conductivity probe was } \\
\text { deployed within the setpoint } \\
\text { limits. }\end{array}$ \\
\hline $\mathrm{F}$ & 02 & South & (A) & 03/15/02 & WAP & / & P02147:01 & Tank condition was normal. \\
\hline $\mathrm{F}$ & 02 & West & (A) & 03/15/02 & WAP & I & P02147:02 & Tank condition was normal. \\
\hline $\mathrm{F}$ & 02 & 05 & (I) & 09/29/02 & CCTV & l & 860 & $\begin{array}{l}\text { Inspection revealed that the } \\
\text { HLLCP was positioned above } \\
\text { the waste. The reel tape and } \\
\text { HLLCP were wrapped around } \\
\text { pipes installed in the riser. }\end{array}$ \\
\hline
\end{tabular}

$(\mathrm{A})$ = annulus; $\mathrm{BFV}=$ back flush valve $\mathrm{CCTV}=$ closed circuit television; $\mathrm{CCWS}=$ chromate cooling water system; $\mathrm{COP}=$ clean out port; $\mathrm{CT}=$ Catch Tank; CTS = concentrate transfer system; CWT = concentrated waste tank; DB = diversion box; DP = direct photography; ERIP = encasement riser inspection port; ETF = effluent treatment facility; EVAP = evaporator; GDL = gravity drain line; HELIUM = helium leak test; HLLCP = high liquid level conductivity probe; $(\mathrm{I})=$ interior; ITPFC = in-tank precipitation filter cell; JB = junction box; LDB = leak detection box; LPPP = low point pump pit; LPS = leak probe sleeve; $M L D B=$ modified leak detection box; PP = pump pit; PSP = periscopic photography; RCP = reinforced concrete pipe; SWS = storm water sewer; UT = ultrasonic test; VB = valve box; VP = video photograph; WAP = wide angle photography; WLE = waste line encasement 


\begin{tabular}{|c|c|c|c|c|c|c|c|c|}
\hline \multirow{2}{*}{$\frac{\text { AREA }}{F}$} & \multirow{2}{*}{$\begin{array}{c}\begin{array}{c}\text { TANK OR } \\
\text { ANCILLARY }\end{array} \\
02\end{array}$} & \multicolumn{2}{|c|}{$\frac{\text { ACCESS OPENING }}{(\text { A OR I) }}$} & \multirow{2}{*}{$\frac{\text { DATE }}{10 / 09 / 02}$} & \multicolumn{3}{|c|}{$\frac{\text { INSPECTION METHOD }}{\text { IDENTIFICATION NUMBER }}$} & \multirow[b]{2}{*}{$\begin{array}{l}\text { REMARKS } \\
\text { CCTV was used to facilitate } \\
\text { flushing and untangling of reel } \\
\text { tape and HLLCP from installed } \\
\text { piping. The reel tape was } \\
\text { flushed, untangled and returned } \\
\text { to service. It was free of } \\
\text { obstructions and was positioned } \\
\text { above a pool of liquid. The } \\
\text { HLLCP was untangled; however, } \\
\text { the probe insulator was } \\
\text { contacting the piping. The probe } \\
\text { was returned to service. }\end{array}$} \\
\hline & & 05 & (I) & & CCTV & / & 875 & \\
\hline $\mathrm{F}$ & 02 & 05 & (I) & $10 / 22 / 02$ & CCTV & I & 875 & $\begin{array}{l}\text { CCTV was used to deploy a new } \\
\text { HLLCP. The HLLCP was } \\
\text { properly positioned, and no } \\
\text { obstructions were observed } \\
\text { beneath the probe. Salt } \\
\text { formations were observed on the } \\
\text { bottom of the reel tape. }\end{array}$ \\
\hline $\mathrm{F}$ & 03 & East & (A) & 03/16/02 & WAP & / & P02146:03 & Tank condition was normal. \\
\hline $\mathrm{F}$ & 03 & North & (A) & 03/06/02 & CCTV & l & 808B & $\begin{array}{l}\text { The conductivity probe was } \\
\text { deployed within the setpoint } \\
\text { limits. }\end{array}$ \\
\hline $\mathrm{F}$ & 03 & North & (A) & 03/16/02 & WAP & / & P02146:02 & Tank condition was normal. \\
\hline $\mathrm{F}$ & 03 & South & $(A)$ & 03/05/02 & DP & l & P02105:01-22 & Tank condition was normal. \\
\hline $\mathrm{F}$ & 03 & South & $(A)$ & 03/06/02 & CCTV & I & 808B & $\begin{array}{l}\text { The conductivity probe was } \\
\text { deployed within the setpoint } \\
\text { limits. }\end{array}$ \\
\hline $\mathrm{F}$ & 03 & West & (A) & 03/06/02 & CCTV & l & 808B & $\begin{array}{l}\text { The magnetically mounted } \\
\text { thermocouple was deployed } \\
\text { within the setpoint limits. }\end{array}$ \\
\hline $\mathrm{F}$ & 03 & West & (A) & $03 / 15 / 02$ & WAP & / & P02146:01 & Tank condition was normal. \\
\hline $\mathrm{F}$ & 04 & East & (A) & 03/06/02 & CCTV & I & 808B & $\begin{array}{l}\text { The magnetically mounted } \\
\text { thermocouple was deployed } \\
\text { within the setpoint limits. }\end{array}$ \\
\hline $\mathrm{F}$ & 04 & East & (A) & 03/06/02 & CCTV & l & 808B & $\begin{array}{l}\text { The magnetically mounted } \\
\text { thermocouple was deployed } \\
\text { within the setpoint limits. }\end{array}$ \\
\hline $\mathrm{F}$ & 04 & East & (A) & $03 / 15 / 02$ & WAP & / & $P 02149: 03$ & Tank condition was normal. \\
\hline $\mathrm{F}$ & 04 & North & (A) & 03/06/02 & CCTV & / & 808B & $\begin{array}{l}\text { The conductivity probe was } \\
\text { deployed within the setpoint } \\
\text { limits. }\end{array}$ \\
\hline
\end{tabular}




\begin{tabular}{|c|c|c|c|c|c|c|c|c|}
\hline \multirow{2}{*}{$\frac{\text { AREA }}{F}$} & \multirow{2}{*}{$\begin{array}{c}\text { TANK OR } \\
\text { ANCILLARY } \\
04\end{array}$} & \multicolumn{2}{|c|}{$\frac{\text { ACCESS OPENING }}{\text { (A OR I) }}$} & \multirow{2}{*}{$\frac{\text { DATE }}{03 / 15 / 02}$} & \multicolumn{3}{|c|}{$\begin{array}{l}\text { INSPECTION METHOD } \\
\text { IDENTIFICATION NUMBER }\end{array}$} & \multirow{2}{*}{$\frac{\text { REMARKS }}{\text { Tank condition was normal. }}$} \\
\hline & & North & (A) & & WAP & / & P02149:02 & \\
\hline $\mathrm{F}$ & 04 & South & (A) & 03/06/02 & CCTV & / & $808 \mathrm{~B}$ & $\begin{array}{l}\text { The conductivity probe was } \\
\text { deployed within the setpoint } \\
\text { limits. }\end{array}$ \\
\hline $\mathrm{F}$ & 04 & South & (A) & $10 / 25 / 02$ & WAP & / & P02236:01 & Tank condition was normal. \\
\hline $\mathrm{F}$ & 04 & West & (A) & 03/05/02 & DP & / & P02106:01-22 & Tank condition was normal. \\
\hline $\mathrm{F}$ & 05 & East & (A) & 03/15/02 & WAP & I & P02144:01 & Tank condition had not changed. \\
\hline $\mathrm{F}$ & 05 & North & (A) & 03/05/02 & DP & / & P02102:01-22 & Tank condition had not changed. \\
\hline $\mathrm{F}$ & 05 & North & (A) & 03/06/02 & CCTV & l & 808B & $\begin{array}{l}\text { The conductivity probe was } \\
\text { deployed within the setpoint } \\
\text { limits. }\end{array}$ \\
\hline $\mathrm{F}$ & 05 & South & (A) & 03/05/02 & DP & / & P02100:01-23 & Tank condition had not changed. \\
\hline $\mathrm{F}$ & 05 & South & (A) & 03/06/02 & CCTV & l & $808 B$ & $\begin{array}{l}\text { The conductivity probe was } \\
\text { deployed within the setpoint } \\
\text { limits. }\end{array}$ \\
\hline $\mathrm{F}$ & 05 & South & (A) & 03/21/02 & CCTV & I & $808 \mathrm{C}$ & $\begin{array}{l}\text { The conductivity probe was } \\
\text { deployed within the setpoint } \\
\text { limits. }\end{array}$ \\
\hline $\mathrm{F}$ & 05 & West & (A) & 03/05/02 & DP & / & P02101:01-22 & Tank condition had not changed. \\
\hline $\mathrm{F}$ & 05 & West & (A) & 03/05/02 & DP & I & P02101:19 & $\begin{array}{l}\text { The magnetically mounted } \\
\text { thermocouple was deployed } \\
\text { within the setpoint limits. }\end{array}$ \\
\hline $\mathrm{F}$ & 06 & East & (A) & 03/19/02 & DP & l & P02097:01-14 & $\begin{array}{l}\text { Inspection was performed to } \\
\text { monitor the leaked waste in the } \\
\text { annulus. Tank condition had not } \\
\text { changed. }\end{array}$ \\
\hline $\mathrm{F}$ & 06 & East & (A) & 03/20/02 & WAP & / & P02103:01 & Tank condition had not changed. \\
\hline $\mathrm{F}$ & 06 & East & (A) & 03/21/02 & CCTV & / & $808 \mathrm{C}$ & $\begin{array}{l}\text { The magnetically mounted } \\
\text { thermocouple was deployed } \\
\text { within the setpoint limits. }\end{array}$ \\
\hline $\mathrm{F}$ & 06 & East & (A) & 08/09/02 & CCTV & I & 840 & $\begin{array}{l}\text { Inspection was performed to } \\
\text { monitor the leaked waste in the } \\
\text { annulus. Tank condition had not } \\
\text { changed. }\end{array}$ \\
\hline $\mathrm{F}$ & 06 & North & (A) & 02/08/02 & DP & 1 & P02066:01-22 & Tank condition had not changed. \\
\hline
\end{tabular}




\begin{tabular}{|c|c|c|c|c|c|c|c|c|}
\hline \multirow{2}{*}{$\frac{\text { AREA }}{F}$} & \multirow{2}{*}{$\begin{array}{c}\begin{array}{c}\text { TANK OR } \\
\text { ANCILLARY }\end{array} \\
06\end{array}$} & \multicolumn{2}{|c|}{$\frac{\text { ACCESS OPENING }}{(\text { A OR I) }}$} & \multirow{2}{*}{$\frac{\text { DATE }}{03 / 20 / 02}$} & \multicolumn{3}{|c|}{$\begin{array}{l}\text { INSPECTION METHOD } \\
\text { IDENTIFICATION NUMBER }\end{array}$} & \multirow{2}{*}{$\begin{array}{l}\text { REMARKS } \\
\text { Inspection was performed to } \\
\text { monitor the leaked waste in the } \\
\text { annulus. Tank condition had not } \\
\text { changed. }\end{array}$} \\
\hline & & North & (A) & & DP & I & P02098:01-13 & \\
\hline $\mathrm{F}$ & 06 & North & (A) & 03/21/02 & CCTV & I & $808 \mathrm{C}$ & $\begin{array}{l}\text { The conductivity probe was } \\
\text { deployed within the setpoint } \\
\text { limits. }\end{array}$ \\
\hline $\mathrm{F}$ & 06 & North & (A) & 08/09/02 & CCTV & l & 840 & $\begin{array}{l}\text { Inspection was performed to } \\
\text { monitor the leaked waste in the } \\
\text { annulus. Tank condition had not } \\
\text { changed. }\end{array}$ \\
\hline $\mathrm{F}$ & 06 & South & (A) & $01 / 13 / 02$ & $\mathrm{DP}$ & l & P02087:01-13 & $\begin{array}{l}\text { Inspection was performed to } \\
\text { monitor the leaked waste in the } \\
\text { annulus. Tank condition had not } \\
\text { changed. }\end{array}$ \\
\hline $\mathrm{F}$ & 06 & South & (A) & 02/08/02 & $\mathrm{DP}$ & 1 & P02065:01-22 & Tank condition had not changed. \\
\hline $\mathrm{F}$ & 06 & South & (A) & 03/19/02 & $\mathrm{DP}$ & l & P02099:01-14 & $\begin{array}{l}\text { Inspection was performed to } \\
\text { monitor the leaked waste in the } \\
\text { annulus. Tank condition had not } \\
\text { changed. }\end{array}$ \\
\hline $\mathrm{F}$ & 06 & South & (A) & 03/21/02 & CCTV & / & $808 \mathrm{C}$ & $\begin{array}{l}\text { The conductivity probe was } \\
\text { deployed within the setpoint } \\
\text { limits. }\end{array}$ \\
\hline $\mathrm{F}$ & 06 & South & (A) & $04 / 11 / 02$ & $\mathrm{DP}$ & l & P02143:01-14 & $\begin{array}{l}\text { Inspection was performed to } \\
\text { monitor the leaked waste in the } \\
\text { annulus. Tank condition had not } \\
\text { changed. }\end{array}$ \\
\hline $\mathrm{F}$ & 06 & South & (A) & 05/10/02 & $\mathrm{DP}$ & l & P02128:01-14 & $\begin{array}{l}\text { Inspection was performed to } \\
\text { monitor leaked waste in the } \\
\text { annulus. Tank condition had not } \\
\text { changed. }\end{array}$ \\
\hline $\mathrm{F}$ & 06 & South & (A) & $06 / 14 / 02$ & $\mathrm{DP}$ & l & P02164:01-12 & $\begin{array}{l}\text { Inspection was performed to } \\
\text { monitor the leaked waste in the } \\
\text { annulus. Tank condition had not } \\
\text { changed. }\end{array}$ \\
\hline $\mathrm{F}$ & 06 & South & (A) & 07/27/02 & $\mathrm{DP}$ & I & P02182:01-26 & $\begin{array}{l}\text { Inspection was performed to } \\
\text { monitor the leaked waste in the } \\
\text { annulus. Tank condition had not } \\
\text { changed. }\end{array}$ \\
\hline $\mathrm{F}$ & 06 & South & (A) & 08/09/02 & CCTV & I & 840 & $\begin{array}{l}\text { Inspection was performed to } \\
\text { monitor the leaked waste in the } \\
\text { annulus. Tank condition had not } \\
\text { changed. }\end{array}$ \\
\hline
\end{tabular}




\begin{tabular}{|c|c|c|c|c|c|c|c|c|}
\hline \multirow{2}{*}{$\frac{\text { AREA }}{F}$} & \multirow{2}{*}{$\begin{array}{c}\text { TANK OR } \\
\text { ANCILLARY } \\
06\end{array}$} & \multicolumn{2}{|c|}{$\frac{\text { ACCESS OPENING }}{(\text { A OR I) }}$} & \multirow{2}{*}{$\frac{\text { DATE }}{04 / 11 / 02}$} & \multicolumn{3}{|c|}{ 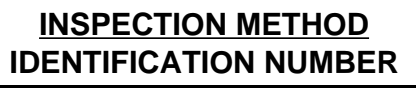 } & \multirow[b]{2}{*}{$\begin{array}{l}\text { REMARKS } \\
\text { Inspection was performed to } \\
\text { monitor the leaked waste in the } \\
\text { annulus. Tank condition had not } \\
\text { changed. Water had entered the } \\
\text { annulus and redistributed the } \\
\text { leaked waste on the annulus } \\
\text { floor causing the appearance of } \\
\text { the deposits on the ventilation } \\
\text { duct and the secondary vessel } \\
\text { wall to change. }\end{array}$} \\
\hline & & West & (A) & & DP & / & P02142:1-15 & \\
\hline $\mathrm{F}$ & 06 & West & (A) & 05/10/02 & DP & / & P02129:01-27 & Tank condition had not changed. \\
\hline $\mathrm{F}$ & 06 & West & (A) & 06/14/02 & DP & / & P02165:01-12 & $\begin{array}{l}\text { Inspection was performed to } \\
\text { monitor the leaked waste in the } \\
\text { annulus. Tank condition had not } \\
\text { changed. }\end{array}$ \\
\hline $\mathrm{F}$ & 06 & West & (A) & 07/12/02 & DP & I & P02177:01-12 & $\begin{array}{l}\text { Inspection was performed to } \\
\text { monitor the leaked waste in the } \\
\text { annulus. Tank condition had not } \\
\text { changed. Water had entered the } \\
\text { annulus and redistributed the } \\
\text { leaked waste on the annulus } \\
\text { floor. }\end{array}$ \\
\hline $\mathrm{F}$ & 06 & West & (A) & 08/09/02 & CCTV & / & 840 & $\begin{array}{l}\text { Inspection was performed to } \\
\text { monitor the leaked waste in the } \\
\text { annulus. Tank condition had not } \\
\text { changed. Water had entered the } \\
\text { annulus and redistributed the } \\
\text { leaked waste on the annulus } \\
\text { floor. }\end{array}$ \\
\hline $\mathrm{F}$ & 06 & Center & (I) & $06 / 15 / 02$ & CCTV & / & 840 & $\begin{array}{l}\text { Inspection documented the } \\
\text { condition of the hydrogen sample } \\
\text { tubes. The tubes were rounded } \\
\text { at the ends. No unusual } \\
\text { conditions were observed. }\end{array}$ \\
\hline $\mathrm{F}$ & 07 & LDB-01 & & 06/18/02 & CCTV & I & 841 & $\begin{array}{l}\text { Inspection revealed that the } \\
\text { standpipe was free of } \\
\text { obstructions. No unusual } \\
\text { conditions were observed. }\end{array}$ \\
\hline $\mathrm{F}$ & 07 & LDB-02 & & 04/29/02 & CCTV & / & $809 F$ & $\begin{array}{l}\text { CCTV was used to document } \\
\text { conditions of the LDB. The floor } \\
\text { of the LDB was damp. }\end{array}$ \\
\hline $\mathrm{F}$ & 07 & LDB-02 & & 06/18/02 & CCTV & I & 841 & $\begin{array}{l}\text { Inspection revealed that the } \\
\text { standpipe was free of } \\
\text { obstructions. No unusual } \\
\text { conditions were observed. }\end{array}$ \\
\hline $\mathrm{F}$ & 07 & VB-01 & & $10 / 28 / 02$ & CCTV & / & 875 & $\begin{array}{l}\text { Valve box conditions were } \\
\text { normal. }\end{array}$ \\
\hline
\end{tabular}




\begin{tabular}{|c|c|c|c|c|c|c|c|}
\hline \multirow{2}{*}{$\frac{\text { AREA }}{F}$} & \multirow{2}{*}{$\begin{array}{c}\begin{array}{c}\text { TANK OR } \\
\text { ANCILLARY }\end{array} \\
07\end{array}$} & $\frac{\text { ACCESS OPENING }}{\text { (A OR I) }}$ & \multirow{2}{*}{$\frac{\text { DATE }}{10 / 28 / 02}$} & \multicolumn{3}{|c|}{$\frac{\text { INSPECTION METHOD }}{\text { IDENTIFICATION NUMBER }}$} & \multirow[b]{2}{*}{$\begin{array}{l}\text { REMARKS } \\
\text { Valve box conditions were } \\
\text { normal. Stains observed on the } \\
\text { stainless steel liner were caused } \\
\text { by the inleakage of water. A } \\
\text { piece of Tygon tubing } \\
\text { approximately } 5 \text { - } 6 \text { feet long was } \\
\text { observed on the bottom of the } \\
\text { valve box. }\end{array}$} \\
\hline & & VB-02 & & CCTV & / & 875 & \\
\hline $\mathrm{F}$ & 07 & VB-03 & $10 / 28 / 02$ & CCTV & l & 875 & $\begin{array}{l}\text { Valve box conditions were } \\
\text { normal. Stains observed on the } \\
\text { stainless steel liner were caused } \\
\text { by the inleakage of water. }\end{array}$ \\
\hline $\mathrm{F}$ & 07 & VB-04 & 08/10/02 & CCTV & l & $809 \mathrm{H}$ & $\begin{array}{l}\text { CCTV was used to investigate a } \\
\text { conductivity probe alarm. } \\
\text { Condensation had caused water } \\
\text { to accumulate on the floor. The } \\
\text { water was removed and the } \\
\text { probe was properly repositioned. }\end{array}$ \\
\hline $\mathrm{F}$ & 07 & VB-04 & $10 / 28 / 02$ & CCTV & l & 875 & $\begin{array}{l}\text { Valve box conditions were } \\
\text { normal. }\end{array}$ \\
\hline $\mathrm{F}$ & 07 & VB-05 & $10 / 28 / 02$ & CCTV & l & 875 & $\begin{array}{l}\text { Valve box conditions were } \\
\text { normal. Stains observed on the } \\
\text { stainless steel liner were caused } \\
\text { by the inleakage of water. }\end{array}$ \\
\hline $\mathrm{F}$ & 07 & North (A) & 02/08/02 & CCTV & / & 809B & $\begin{array}{l}\text { Inspection was made to } \\
\text { document the conditions in the } \\
\text { annulus prior to starting waste } \\
\text { removal activities. The annulus } \\
\text { floor was dry, and no unusual } \\
\text { conditions were observed. }\end{array}$ \\
\hline $\mathrm{F}$ & 07 & North $(A)$ & 02/08/02 & CCTV & l & $809 A$ & $\begin{array}{l}\text { The conductivity probe was } \\
\text { deployed within the setpoint } \\
\text { limits. }\end{array}$ \\
\hline $\mathrm{F}$ & 07 & North $(A)$ & 02/08/02 & $\mathrm{DP}$ & l & P02121:01-22 & Tank condition was normal. \\
\hline $\mathrm{F}$ & 07 & South $(A)$ & 02/08/02 & CCTV & I & 809B & $\begin{array}{l}\text { Inspection was made to } \\
\text { document the conditions in the } \\
\text { annulus prior to starting waste } \\
\text { removal activities. The annulus } \\
\text { floor was dry, and no unusual } \\
\text { conditions were observed. }\end{array}$ \\
\hline $\mathrm{F}$ & 07 & South $(A)$ & 02/08/02 & CCTV & l & $809 A$ & $\begin{array}{l}\text { The conductivity probe was } \\
\text { deployed within the setpoint } \\
\text { limits. }\end{array}$ \\
\hline $\mathrm{F}$ & 07 & South $(A)$ & 02/08/02 & $\mathrm{DP}$ & / & P02120:01-22 & Tank condition was normal. \\
\hline
\end{tabular}




\begin{tabular}{|c|c|c|c|c|c|c|c|c|}
\hline \multirow{2}{*}{$\frac{\text { AREA }}{F}$} & \multirow{2}{*}{$\begin{array}{c}\begin{array}{c}\text { TANK OR } \\
\text { ANCILLARY }\end{array} \\
07\end{array}$} & \multicolumn{2}{|c|}{$\frac{\text { ACCESS OPENING }}{(\text { A OR I) }}$} & \multirow{2}{*}{$\frac{\text { DATE }}{02 / 08 / 02}$} & \multicolumn{3}{|c|}{$\begin{array}{l}\text { INSPECTION METHOD } \\
\text { IDENTIFICATION NUMBER }\end{array}$} & \multirow{2}{*}{$\begin{array}{l}\text { REMARKS } \\
\text { Inspection was made to } \\
\text { document the conditions in the } \\
\text { annulus prior to starting waste } \\
\text { removal activities. The annulus } \\
\text { floor was dry, and no unusual } \\
\text { conditions were observed. }\end{array}$} \\
\hline & & West & $(A)$ & & CCTV & / & 809B & \\
\hline $\mathrm{F}$ & 07 & West & $(A)$ & 02/08/02 & CCTV & / & $809 \mathrm{~A}$ & $\begin{array}{l}\text { The magnetically mounted } \\
\text { thermocouple was deployed } \\
\text { within the setpoint limits. }\end{array}$ \\
\hline $\mathrm{F}$ & 07 & West & $(A)$ & 02/08/02 & DP & l & P02119:01-22 & Tank condition was normal. \\
\hline $\mathrm{F}$ & 07 & 04 & (I) & $07 / 25 / 02$ & CCTV & / & 840 & $\begin{array}{l}\text { Inspection verified that the } \\
\text { conductivity probe was properly } \\
\text { positioned in the spray chamber. }\end{array}$ \\
\hline $\mathrm{F}$ & 07 & 04 & (I) & 08/22/02 & CCTV & / & 860 & $\begin{array}{l}\text { Inspection verified that the } \\
\text { conductivity probe was properly } \\
\text { positioned in the spray chamber. }\end{array}$ \\
\hline $\mathrm{F}$ & 07 & Center & (I) & $07 / 21 / 02$ & CCTV & l & 840 & $\begin{array}{l}\text { Inspection verified that the } \\
\text { bearing water columns were full } \\
\text { in the slurry pumps installed in } \\
\text { risers } 1,3,5 \text { and } 8 \text {. }\end{array}$ \\
\hline $\mathrm{F}$ & 07 & Center & $(\mathrm{l})$ & $11 / 02 / 02$ & CCTV & / & 875 & $\begin{array}{l}\text { CCTV was used to document } \\
\text { conditions of the HLLCP, the reel } \\
\text { tape and the tank contents. The } \\
\text { HLLCP was unobstructed and } \\
\text { properly positioned. The reel } \\
\text { tape was unobstructed but was } \\
\text { coated with salt. Liquid was } \\
\text { observed throughout the tank. }\end{array}$ \\
\hline $\mathrm{F}$ & 08 & East & $(A)$ & 03/15/02 & WAP & / & P02145:03 & Tank condition was normal. \\
\hline $\mathrm{F}$ & 08 & East & (A) & 03/21/02 & CCTV & / & $808 \mathrm{C}$ & $\begin{array}{l}\text { The magnetically mounted } \\
\text { thermocouple was deployed } \\
\text { within the setpoint limits. }\end{array}$ \\
\hline $\mathrm{F}$ & 08 & North & $(A)$ & 03/15/02 & $\mathrm{DP}$ & / & P02148:01-21 & Tank condition was normal. \\
\hline $\mathrm{F}$ & 08 & North & (A) & 03/21/02 & CCTV & / & $808 C$ & $\begin{array}{l}\text { The conductivity probe was } \\
\text { deployed within the setpoint } \\
\text { limits. }\end{array}$ \\
\hline $\mathrm{F}$ & 08 & South & $(A)$ & 03/15/02 & WAP & / & P02145:02 & Tank condition was normal. \\
\hline $\mathrm{F}$ & 08 & South & $(\mathrm{A})$ & 03/21/02 & CCTV & / & $808 \mathrm{C}$ & $\begin{array}{l}\text { The conductivity probe was } \\
\text { deployed within the setpoint } \\
\text { limits. }\end{array}$ \\
\hline $\mathrm{F}$ & 08 & West & $(A)$ & 03/15/02 & WAP & / & P02145:01 & Tank condition was normal. \\
\hline
\end{tabular}




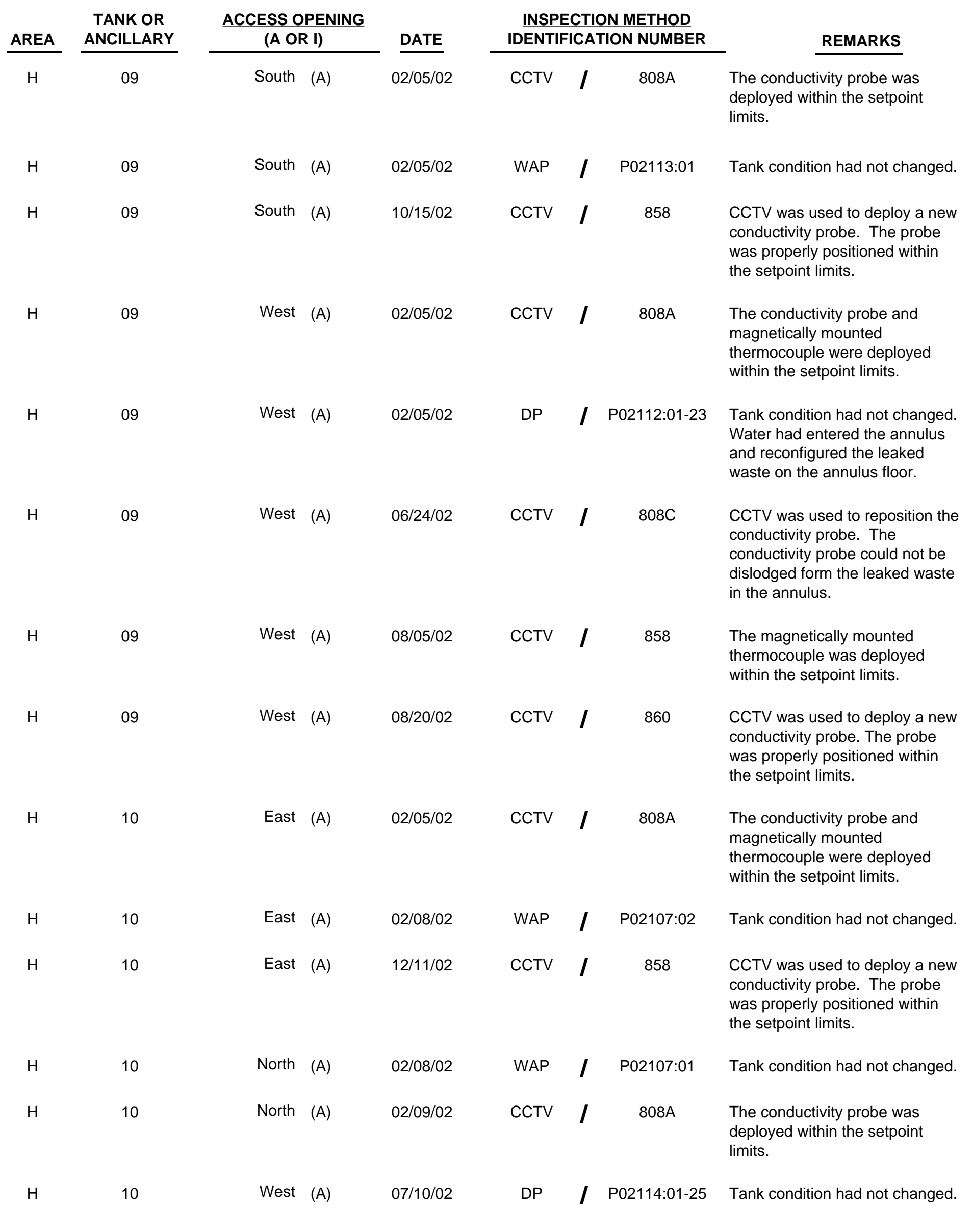




\begin{tabular}{|c|c|c|c|c|c|c|c|c|}
\hline \multirow{2}{*}{$\frac{\text { AREA }}{\mathrm{H}}$} & \multirow{2}{*}{$\begin{array}{c}\begin{array}{c}\text { TANK OR } \\
\text { ANCILLARY }\end{array} \\
11\end{array}$} & \multicolumn{2}{|c|}{$\frac{\text { ACCESS OPENING }}{(\text { A OR I) }}$} & \multirow{2}{*}{$\frac{\text { DATE }}{11 / 27 / 02}$} & \multicolumn{3}{|c|}{$\begin{array}{l}\text { INSPECTION METHOD } \\
\text { IDENTIFICATION NUMBER }\end{array}$} & \multirow{2}{*}{$\frac{\text { REMARKS }}{\text { Tank condition had not changed. }}$} \\
\hline & & East & $(A)$ & & DP & l & P02241:01-21 & \\
\hline $\mathrm{H}$ & 11 & North & (A) & 02/09/02 & CCTV & / & $808 \mathrm{~A}$ & $\begin{array}{l}\text { The conductivity probe was } \\
\text { deployed within the setpoint } \\
\text { limits. }\end{array}$ \\
\hline $\mathrm{H}$ & 11 & North & $(\mathrm{A})$ & 09/05/02 & DP & / & P02224:01-22 & Tank condition had not changed. \\
\hline $\mathrm{H}$ & 11 & South & (A) & 02/09/02 & CCTV & / & $808 \mathrm{~A}$ & $\begin{array}{l}\text { The conductivity probe was } \\
\text { deployed within the setpoint } \\
\text { limits. }\end{array}$ \\
\hline $\mathrm{H}$ & 11 & South & $(A)$ & 02/09/02 & WAP & l & P02126:01 & Tank condition had not changed. \\
\hline $\mathrm{H}$ & 11 & South & (A) & 05/18/02 & CCTV & / & $808 \mathrm{C}$ & $\begin{array}{l}\text { CCTV was used to investigate } \\
\text { spurious conductivity probe } \\
\text { alarms. No unusual conditions } \\
\text { were observed. }\end{array}$ \\
\hline $\mathrm{H}$ & 11 & South & (A) & 05/20/02 & CCTV & / & $808 \mathrm{C}$ & $\begin{array}{l}\text { CCTV was used to investigate } \\
\text { spurious conductivity probe } \\
\text { alarms. No liquid was observed; } \\
\text { however, a steel cable was } \\
\text { contacting the probe which may } \\
\text { provide a path to ground. }\end{array}$ \\
\hline $\mathrm{H}$ & 11 & West & $(A)$ & 02/09/02 & WAP & / & P02126:02 & Tank condition had not changed. \\
\hline $\mathrm{H}$ & 11 & West & $(A)$ & 02/09/02 & CCTV & / & $808 \mathrm{~A}$ & $\begin{array}{l}\text { The magnetically mounted } \\
\text { thermocouple was deployed } \\
\text { within the setpoint limits. }\end{array}$ \\
\hline $\mathrm{H}$ & 12 & East & $(A)$ & $02 / 15 / 02$ & CCTV & I & 808B & $\begin{array}{l}\text { The magnetically mounted } \\
\text { thermocouple was deployed } \\
\text { within the setpoint limits. }\end{array}$ \\
\hline $\mathrm{H}$ & 12 & East & (A) & $02 / 15 / 02$ & WAP & / & P02117:02 & Tank condition had not changed. \\
\hline $\mathrm{H}$ & 12 & North & (A) & $02 / 15 / 02$ & CCTV & I & 808B & $\begin{array}{l}\text { The conductivity probe was } \\
\text { deployed within the setpoint } \\
\text { limits. }\end{array}$ \\
\hline $\mathrm{H}$ & 12 & North & $(A)$ & $07 / 11 / 02$ & DP & l & P02116:01-24 & Tank condition had not changed. \\
\hline $\mathrm{H}$ & 12 & North & $(\mathrm{A})$ & 08/28/02 & CCTV & I & 858 & $\begin{array}{l}\text { Inspection was performed to } \\
\text { investigate a possible change } \\
\text { noted during a scheduled } \\
\text { inspection on } 7 / 11 / 02 \text { in } \\
\text { previously identified leaksites. } \\
\text { No change had occurred. }\end{array}$ \\
\hline $\mathrm{H}$ & 12 & South & $(A)$ & $02 / 15 / 02$ & CCTV & l & 808B & $\begin{array}{l}\text { The conductivity probe was } \\
\text { deployed within the setpoint } \\
\text { limits. }\end{array}$ \\
\hline
\end{tabular}




\begin{tabular}{|c|c|c|c|c|c|c|c|c|}
\hline \multirow{2}{*}{$\frac{\text { AREA }}{\mathrm{H}}$} & \multirow{2}{*}{$\begin{array}{c}\begin{array}{c}\text { TANK OR } \\
\text { ANCILLARY }\end{array} \\
12\end{array}$} & \multicolumn{2}{|c|}{$\frac{\text { ACCESS OPENING }}{(\text { A OR I) }}$} & \multirow{2}{*}{$\frac{\text { DATE }}{02 / 15 / 02}$} & \multicolumn{3}{|c|}{$\begin{array}{l}\text { INSPECTION METHOD } \\
\text { IDENTIFICATION NUMBER }\end{array}$} & \multirow[b]{2}{*}{ Tank condition had not changed. } \\
\hline & & South & (A) & & WAP & / & P02117:01 & \\
\hline $\mathrm{H}$ & 12 & West & (A) & 09/05/02 & DP & I & P02225:01-22 & Tank condition had not changed. \\
\hline $\mathrm{H}$ & 13 & LDB-02 & & 03/05/02 & CCTV & I & $807 \mathrm{~A}$ & $\begin{array}{l}\text { The conductivity probe was } \\
\text { deployed within the setpoint } \\
\text { limits. }\end{array}$ \\
\hline $\mathrm{H}$ & 13 & 010 & (A) & $02 / 14 / 02$ & DP & I & P02090:01-22 & Tank condition had not changed. \\
\hline $\mathrm{H}$ & 13 & 032 & (A) & $02 / 14 / 02$ & DP & / & P02091:01-22 & Tank condition had not changed. \\
\hline $\mathrm{H}$ & 13 & 055 & (A) & 02/14/02 & DP & I & P02092:01-22 & Tank condition had not changed. \\
\hline $\mathrm{H}$ & 13 & 071 & (A) & 02/14/02 & DP & / & P02093:01-22 & Tank condition had not changed \\
\hline $\mathrm{H}$ & 13 & 107 & (A) & 02/14/02 & DP & / & P02094:01-22 & Tank condition had not changed. \\
\hline $\mathrm{H}$ & 13 & 151 & (A) & $02 / 14 / 02$ & DP & I & P02095:01-22 & Tank condition had not changed \\
\hline $\mathrm{H}$ & 13 & 175 & (A) & 02/13/02 & WAP & / & P02122:02 & Tank condition had not changed \\
\hline $\mathrm{H}$ & 13 & 207 & (A) & 02/13/02 & WAP & / & P02122:01 & Tank condition had not changed. \\
\hline $\mathrm{H}$ & 13 & 228 & (A) & 02/14/02 & DP & / & P02096:01-22 & Tank condition had not changed. \\
\hline $\mathrm{H}$ & 13 & East & (A) & 02/13/02 & WAP & / & P02122:03 & Tank condition had not changed. \\
\hline $\mathrm{H}$ & 13 & North & (A) & 02/13/02 & CCTV & I & $808 \mathrm{~B}$ & $\begin{array}{l}\text { The conductivity probe and } \\
\text { magnetically mounted } \\
\text { thermocouple were deployed } \\
\text { within the setpoint limits. }\end{array}$ \\
\hline $\mathrm{H}$ & 13 & North & (A) & 06/18/02 & DP & 1 & P02088:01-23 & Tank condition had not changed. \\
\hline $\mathrm{H}$ & 13 & South & (A) & 02/13/02 & CCTV & / & $808 \mathrm{~B}$ & $\begin{array}{l}\text { The conductivity probe was } \\
\text { deployed within the setpoint } \\
\text { limits. }\end{array}$ \\
\hline H & 13 & South & (A) & $02 / 14 / 02$ & DP & 1 & P02089:01-22 & Tank condition had not changed. \\
\hline $\mathrm{H}$ & 13 & West & (A) & 02/13/02 & WAP & / & P02122:04 & Tank condition had not changed. \\
\hline $\mathrm{H}$ & 14 & 013 & (A) & 08/29/02 & WAP & / & P02208:01 & Tank condition had not changed. \\
\hline $\mathrm{H}$ & 14 & 032 & (A) & $11 / 27 / 02$ & WAP & / & P02242:01 & Tank condition had not changed. \\
\hline $\mathrm{H}$ & 14 & 065 & (A) & 08/29/02 & DP & / & P02206:01-22 & Tank condition had not changed. \\
\hline $\mathrm{H}$ & 14 & 108 & (A) & 08/29/02 & DP & 1 & P02204:01-23 & Tank condition had not changed. \\
\hline
\end{tabular}




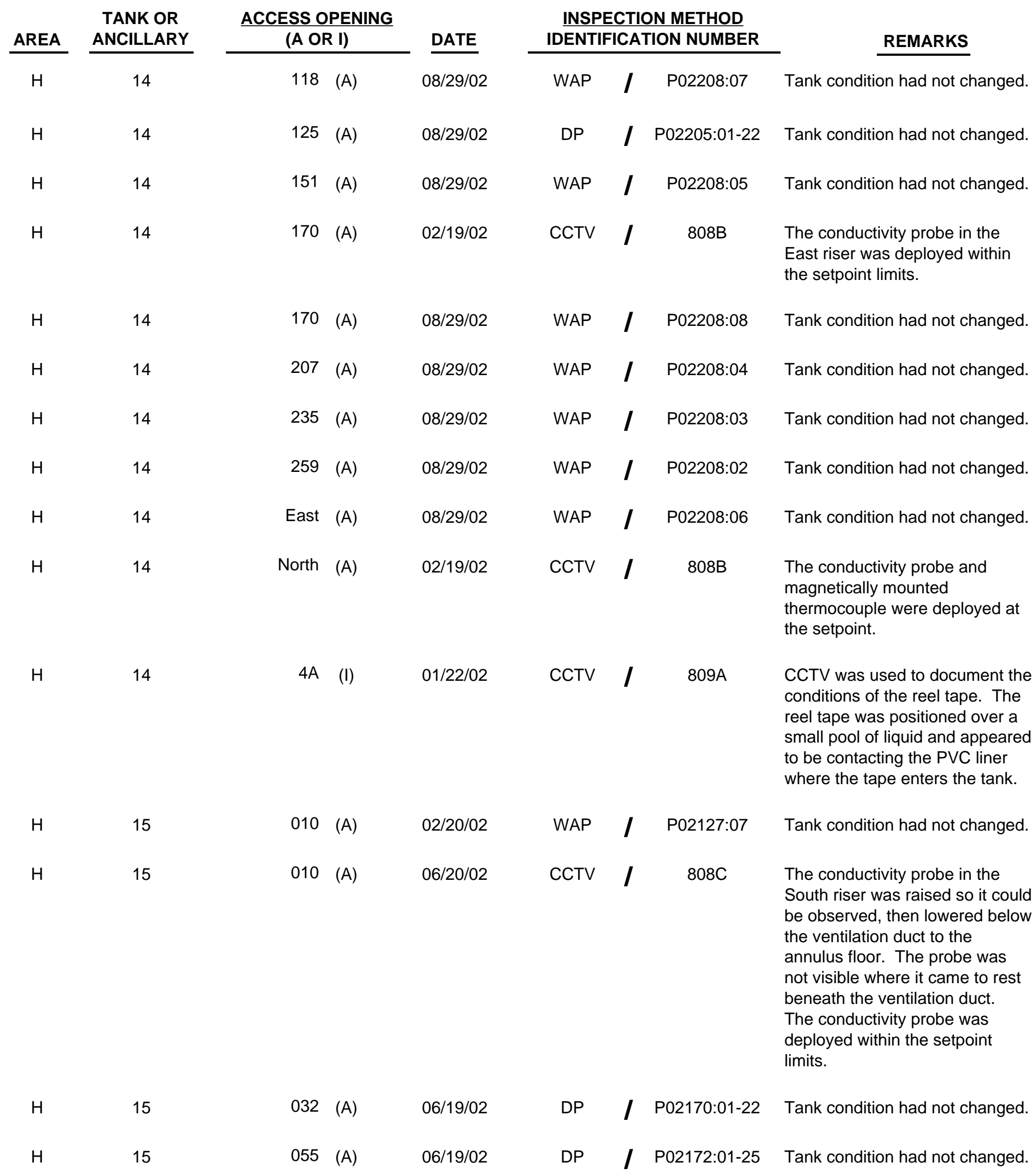




\section{TANK OR \\ AREA}

$\mathrm{H}$

\section{ACCESS OPENING} (A OR I)

055 (A)
INSPECTION METHOD IDENTIFICATION NUMBER

UT ～/ UT-02-001
DATE

08/08/02
REMARKS

Thickness mapping of the tank wall was performed on a vertical strip 8.5 inches wide for the entire accessible height from the top girth weld to the bottom girth weld. Analysis of the data revealed no reportable thinning or pitting. One 0.030 inch wall loss area, which is still above nominal thickness, was noted for future reference. No cracking was detected in the vertical strip. The previously observed crack on the vertical weld 200 inches above the tank bottom was mapped for reference data. This information is included in document

WSRC-TR-2002-00565.

$06 / 19 / 02$

02/20/02

WAP

/ P02173:01-24

Tank condition had not changed.

$\mathrm{H}$ 15

107 (A)

09/05/02

107 (A)

UT

I

P02127:03

UT-02-002

Thickness mapping of the tank wall was performed on a vertical strip 8.5 inches wide for the entire accessible height from the top girth weld to the bottom girth weld. Analysis of the data revealed no reportable thinning or pitting. No cracking was detected in the vertical strip. A crack on a vertical weld 88 inches above the tank bottom was mapped for reference data. It measured 3.7 inches through-wall. This information is included in document WSRC-TR-2002-00565.

$\mathrm{H}$

CCTV

I

$808 C$

$\mathrm{H}$

15

$137 \quad(\mathrm{~A})$

02/20/02

WAP

I

P02127:04

WAP / P02127:05

$02 / 20 / 02$

171 (A)

02/20/02
$182(\mathrm{~A})$
15
WAP
The conductivity probe was deployed within the setpoint limits.

Tank condition had not changed.

Tank condition had not changed.
Tank condition had not changed. 


\section{TANK OR ANCILLARY}

$\mathrm{H}$
15

$\frac{\frac{\text { ACCESS OPENING }}{\text { (A OR I) }}}{182 \text { (A) }} \frac{\text { DATE }}{09 / 17 / 02}$

$\frac{\frac{\text { INSPECTION METHOD }}{\text { IDENTIFICATION NUMBER }}}{\text { UT / UT-02-003 }}$

\section{REMARKS}

Thickness mapping of the tank wall was performed on a vertical strip 8.5 inches wide for the entire accessible height from the top girth weld to the bottom girth weld. Analysis of the data revealed no reportable thinning or pitting. Three partial through-wall cracks were detected at 49, 51, and 139 inches above the tank bottom, and a complete through-wall crack was detected at 129 inches above the tank bottom 7 feet north of the riser. These indications were perpendicular to the vertical weld. An additional partial through-wall crack was detected 31 inches above the tank bottom 1 foot north of the riser. Approximately 34 feet of the middle girth weld was scanned for horizontal and vertical cracking. Three cracks on the middle girth weld were mapped for reference data. A crack 10 feet north of the riser was measured at 7 inches through-wall, a crack 10 feet south of the riser was measured at 10.2 inches through-wall and a crack 25 feet south of the riser was measured at 12.7 inches through-wall. A bottom plate vertical weld was scanned intermittently. This information is included in document WSRC-TR-2002-00565. magnetically mounted thermocouple was deployed within the setpoint limits.
06/19/02

06/19/02

06/20/02

$02 / 20 / 02$
WAP

\section{CCTV / 808C \\ DP \\ DP / P02171:01-23}

r

East (A)

15

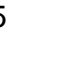

East (A) 02/20/02 WAP / P02127:02 Tank condition had not changed.




\begin{tabular}{|c|c|c|c|c|c|c|c|c|}
\hline \multirow{2}{*}{$\frac{\text { AREA }}{\mathrm{H}}$} & \multirow{2}{*}{$\begin{array}{c}\begin{array}{c}\text { TANK OR } \\
\text { ANCILLARY }\end{array} \\
15\end{array}$} & \multicolumn{2}{|c|}{$\frac{\text { ACCESS OPENING }}{(A \text { OR I) }}$} & \multirow{2}{*}{$\frac{\text { DATE }}{\text { 09/19/02 }}$} & \multicolumn{3}{|c|}{$\begin{array}{l}\text { INSPECTION METHOD } \\
\text { IDENTIFICATION NUMBER }\end{array}$} & \multirow[b]{2}{*}{$\begin{array}{l}\text { REMARKS } \\
\text { Thickness mapping of the tank } \\
\text { wall was performed on a vertical } \\
\text { strip } 8.5 \text { inches wide for the } \\
\text { entire accessible height from the } \\
\text { top girth weld to the bottom girth } \\
\text { weld via riser } 182 \text {. Analysis of } \\
\text { the data revealed no reportable } \\
\text { thinning or pitting. No cracking } \\
\text { was detected in the vertical strip. } \\
\text { This information is included in } \\
\text { document } \\
\text { WSRC-TR-2002-00565. }\end{array}$} \\
\hline & & East & (A) & & UT & / & UT-02-004 & \\
\hline $\mathrm{H}$ & 15 & North & (A) & 09/09/02 & DP & / & P02188:01-22 & Tank condition had not changed. \\
\hline $\mathrm{H}$ & 15 & South & (A) & 02/20/02 & WAP & / & P02127:06 & Tank condition had not changed. \\
\hline $\mathrm{H}$ & 15 & West & (A) & 09/09/02 & DP & / & P02187:01-22 & Tank condition had not changed. \\
\hline $\mathrm{H}$ & 16 & 035 & (A) & $06 / 27 / 02$ & WAP & / & P02181:03 & Tank condition had not changed. \\
\hline $\mathrm{H}$ & 16 & 118 & (A) & 06/27/02 & WAP & / & P02181:04 & Tank condition had not changed. \\
\hline $\mathrm{H}$ & 16 & 207 & (A) & 06/27/02 & WAP & / & P02181:01 & Tank condition had not changed. \\
\hline $\mathrm{H}$ & 16 & 262 & (A) & $06 / 27 / 02$ & WAP & / & P02181:02 & Tank condition had not changed. \\
\hline $\mathrm{H}$ & 16 & East & (A) & 06/26/02 & DP & / & P02180:01-22 & Tank condition had not changed. \\
\hline $\mathrm{H}$ & 16 & West & $(\mathrm{A})$ & 06/26/02 & DP & l & P02179:01-22 & Tank condition had not changed. \\
\hline $\mathrm{F}$ & 18 & MLDB-10 & & 04/03/02 & CCTV & / & $807 \mathrm{~A}$ & $\begin{array}{l}\text { The conductivity probe was } \\
\text { deployed within the setpoint } \\
\text { limits. }\end{array}$ \\
\hline $\mathrm{F}$ & 18 & Center & (I) & $09 / 17 / 02$ & CCTV & / & 870 & $\begin{array}{l}\text { Tank steel wall and concrete } \\
\text { dome were normal. }\end{array}$ \\
\hline $\mathrm{F}$ & 18 & Center & (I) & $10 / 14 / 02$ & CCTV & / & 870 & $\begin{array}{l}\text { Inspection revealed that the } \\
\text { HLLCP was properly deployed. }\end{array}$ \\
\hline$F$ & 18 & Center & (I) & $10 / 15 / 02$ & CCTV & I & 870 & $\begin{array}{l}\text { Inspection revealed that the } \\
\text { HLLCP was properly deployed. }\end{array}$ \\
\hline$F$ & 18 & Center & (I) & $10 / 23 / 02$ & CCTV & I & 878 & $\begin{array}{l}\text { Inspection revealed that the } \\
\text { HLLCP was properly deployed. }\end{array}$ \\
\hline $\mathrm{F}$ & 18 & Center & (I) & $11 / 18 / 02$ & CCTV & I & 878 & $\begin{array}{l}\text { Inspection revealed that the } \\
\text { HLLCP was properly deployed. }\end{array}$ \\
\hline
\end{tabular}




\begin{tabular}{|c|c|c|c|c|c|c|c|c|}
\hline \multirow{2}{*}{$\frac{\text { AREA }}{F}$} & \multirow{2}{*}{$\begin{array}{c}\text { TANK OR } \\
\text { ANCILLARY } \\
18\end{array}$} & \multicolumn{2}{|c|}{$\frac{\text { ACCESS OPENING }}{(\text { A OR I) }}$} & \multirow{2}{*}{$\frac{\text { DATE }}{12 / 11 / 02}$} & \multicolumn{3}{|c|}{$\begin{array}{l}\text { INSPECTION METHOD } \\
\text { IDENTIFICATION NUMBER }\end{array}$} & \multirow[b]{2}{*}{$\begin{array}{l}\text { REMARKS } \\
\text { CCTV was used to monitor the } \\
\text { ADMP and observe for the } \\
\text { buildup of solids on the tank wall. } \\
\text { All deposits, scaling, and } \\
\text { artifacts observed existed prior } \\
\text { to ADMP operations. Foam and } \\
\text { several feet of tubing were } \\
\text { observed floating on the liquid } \\
\text { surface. }\end{array}$} \\
\hline & & Center & (I) & & CCTV & I & 895 & \\
\hline $\mathrm{F}$ & 18 & $\mathrm{NE}$ & (I) & 02/21/02 & CCTV & I & $809 C$ & $\begin{array}{l}\text { Inspection was made to } \\
\text { determine the condition of the } \\
\text { nozzle for the transfer line. No } \\
\text { unusual conditions were } \\
\text { observed. }\end{array}$ \\
\hline $\mathrm{F}$ & 18 & NE & (I) & $11 / 18 / 02$ & CCTV & I & 878 & $\begin{array}{l}\text { Inspection documented the } \\
\text { conditions beneath the riser. } \\
\text { Abandoned hoses were } \\
\text { observed below the riser near } \\
\text { the transfer pump. }\end{array}$ \\
\hline $\mathrm{F}$ & 19 & Center & (I) & $10 / 15 / 02$ & CCTV & I & 877 & $\begin{array}{l}\text { Tank steel wall condition had not } \\
\text { changed, and the concrete dome } \\
\text { was normal. }\end{array}$ \\
\hline $\mathrm{H}$ & 21 & NE & (I) & 05/21/02 & CCTV & I & 833 & $\begin{array}{l}\text { Tank steel wall was normal. } \\
\text { Inspection of the concrete dome } \\
\text { revealed a few surface voids. } \\
\text { The waste surface was covered } \\
\text { with a gray film with small orange } \\
\text { splotches scattered throughout } \\
\text { the tank. The HLLCP was } \\
\text { properly deployed. }\end{array}$ \\
\hline $\mathrm{H}$ & 22 & MLDB-01 & & $05 / 17 / 02$ & CCTV & I & $807 \mathrm{~A}$ & $\begin{array}{l}\text { The conductivity probe was } \\
\text { deployed within the setpoint } \\
\text { limits. }\end{array}$ \\
\hline $\mathrm{H}$ & 22 & NE & (I) & $05 / 17 / 02$ & CCTV & I & 829 & $\begin{array}{l}\text { Tank steel wall and concrete } \\
\text { dome were normal. Stains } \\
\text { observed on the concrete dome } \\
\text { were caused by inleakage of } \\
\text { water. The waste surface was } \\
\text { covered with a gray film with } \\
\text { some observable floating solids. } \\
\text { The HLLCP was properly } \\
\text { deployed. }\end{array}$ \\
\hline
\end{tabular}




\begin{tabular}{|c|c|c|c|c|c|c|c|c|}
\hline \multirow{2}{*}{$\frac{\text { AREA }}{\mathrm{H}}$} & \multirow{2}{*}{$\begin{array}{c}\begin{array}{c}\text { TANK OR } \\
\text { ANCILLARY }\end{array} \\
23\end{array}$} & \multicolumn{2}{|c|}{$\frac{\text { ACCESS OPENING }}{(A \text { OR I) }}$} & \multirow{2}{*}{$\frac{\text { DATE }}{05 / 21 / 02}$} & \multicolumn{3}{|c|}{$\begin{array}{l}\text { INSPECTION METHOD } \\
\text { IDENTIFICATION NUMBER }\end{array}$} & \multirow[b]{2}{*}{$\begin{array}{l}\text { REMARKS } \\
\text { Tank steel wall and concrete } \\
\text { dome were normal. Stains } \\
\text { observed on the concrete dome } \\
\text { were caused by inleakage of } \\
\text { water. The waste surface was } \\
\text { free of solids, and no unusual } \\
\text { conditions were observed. The } \\
\text { HLLCP was properly deployed. }\end{array}$} \\
\hline & & SW & (I) & & CCTV & / & 834 & \\
\hline $\mathrm{H}$ & 24 & SW & (I) & $01 / 26 / 02$ & CCTV & l & $754 \mathrm{~A}$ & $\begin{array}{l}\text { Inspection of the material on the } \\
\text { surface revealed no significant } \\
\text { changes since last inspected on } \\
12 / 9 / 01 \text {. }\end{array}$ \\
\hline $\mathrm{H}$ & 24 & SW & (I) & $02 / 22 / 02$ & CCTV & / & 815 & $\begin{array}{l}\text { Inspection was made to } \\
\text { document the tank waste } \\
\text { surface. The liquid surface was } \\
\text { covered with a gray film with } \\
\text { some brown splotches scattered } \\
\text { throughout the tank. }\end{array}$ \\
\hline $\mathrm{H}$ & 24 & SW & (I) & 05/16/02 & CCTV & l & 815 & $\begin{array}{l}\text { Tank steel wall and concrete } \\
\text { dome were normal. Stains } \\
\text { observed on the concrete dome } \\
\text { were caused by inleakage of } \\
\text { water. The HLLCP was } \\
\text { improperly positioned; it was } \\
\text { resting on the drop valve. The } \\
\text { HLLCP was repositioned and } \\
\text { properly deployed. }\end{array}$ \\
\hline $\mathrm{F}$ & 25 & $A-01$ & (A) & $01 / 29 / 02$ & WAP & / & P02042:02 & Tank condition was normal. \\
\hline $\mathrm{F}$ & 25 & A-02 & (A) & $01 / 29 / 02$ & WAP & / & P02042:10 & Tank condition was normal. \\
\hline $\mathrm{F}$ & 25 & A-02 & (A) & $01 / 31 / 02$ & CCTV & / & $808 \mathrm{~A}$ & $\begin{array}{l}\text { The conductivity probe was } \\
\text { deployed within the setpoint } \\
\text { limits. }\end{array}$ \\
\hline $\mathrm{F}$ & 25 & A-03 & (A) & 01/29/02 & WAP & / & P02042:06 & Tank condition was normal. \\
\hline $\mathrm{F}$ & 25 & A-03 & (A) & 01/31/02 & CCTV & / & $808 \mathrm{~A}$ & $\begin{array}{l}\text { The conductivity probe was } \\
\text { deployed within the setpoint } \\
\text { limits. }\end{array}$ \\
\hline $\mathrm{F}$ & 25 & A-04 & (A) & 01/29/02 & WAP & / & P02042:08 & Tank condition was normal. \\
\hline $\mathrm{F}$ & 25 & A-04 & (A) & $01 / 31 / 02$ & CCTV & l & $808 \mathrm{~A}$ & $\begin{array}{l}\text { The conductivity probe was } \\
\text { deployed within the setpoint } \\
\text { limits. }\end{array}$ \\
\hline $\mathrm{F}$ & 25 & P-01 & (A) & 01/29/02 & WAP & l & P02042:01 & Tank condition was normal. \\
\hline $\mathrm{F}$ & 25 & P-02 & $(\mathrm{A})$ & $01 / 29 / 02$ & WAP & l & P02042:03 & Tank condition was normal. \\
\hline
\end{tabular}




\begin{tabular}{|c|c|c|c|c|c|c|c|c|}
\hline \multirow{2}{*}{$\frac{\text { AREA }}{F}$} & \multirow{2}{*}{$\begin{array}{c}\begin{array}{c}\text { TANK OR } \\
\text { ANCILLARY }\end{array} \\
25\end{array}$} & \multicolumn{2}{|c|}{$\frac{\text { ACCESS OPENING }}{(\text { A OR I) }}$} & \multirow{2}{*}{$\frac{\text { DATE }}{01 / 29 / 02}$} & \multicolumn{3}{|c|}{$\begin{array}{l}\text { INSPECTION METHOD } \\
\text { IDENTIFICATION NUMBER }\end{array}$} & \multirow{2}{*}{$\frac{\text { REMARKS }}{\text { Tank condition was normal. }}$} \\
\hline & & P-03 & (A) & & WAP & / & P02042:04 & \\
\hline $\mathrm{F}$ & 25 & P-04 & (A) & 01/29/02 & WAP & l & P02042:05 & Tank condition was normal. \\
\hline $\mathrm{F}$ & 25 & P-05 & (A) & 01/23/02 & $\mathrm{DP}$ & l & P02009:01-25 & Tank condition was normal. \\
\hline $\mathrm{F}$ & 25 & P-06 & (A) & 01/23/02 & DP & / & P02010:01-25 & Tank condition was normal. \\
\hline $\mathrm{F}$ & 25 & P-07 & (A) & 01/29/02 & WAP & l & P02042:11 & Tank condition was normal. \\
\hline $\mathrm{F}$ & 25 & P-08 & (A) & 01/29/02 & WAP & / & P02042:12 & Tank condition was normal. \\
\hline $\mathrm{F}$ & 25 & P-09 & (A) & 01/29/02 & WAP & / & P02042:13 & Tank condition was normal. \\
\hline $\mathrm{F}$ & 25 & P-10 & (A) & 01/23/02 & DP & / & P02011:01-25 & Tank condition was normal. \\
\hline $\mathrm{F}$ & 25 & $P-11$ & (A) & 01/29/02 & WAP & I & P02042:07 & Tank condition was normal. \\
\hline $\mathrm{F}$ & 25 & P-12 & (A) & $01 / 29 / 02$ & WAP & I & P02042:09 & Tank condition was normal. \\
\hline $\mathrm{F}$ & 25 & $P-13$ & (A) & $01 / 23 / 02$ & DP & l & P02012:01-25 & Tank condition was normal. \\
\hline$F$ & 25 & P-14 & (A) & $01 / 23 / 02$ & DP & l & P02013:01-25 & Tank condition was normal. \\
\hline $\mathrm{F}$ & 25 & B-02 & (I) & 01/10/02 & CCTV & I & 809 & $\begin{array}{l}\text { Inspection was made to } \\
\text { document the conditions of the } \\
\text { waste surface and to verify that } \\
\text { the reel tape was functioning } \\
\text { properly. The salt cake was } \\
\text { covered with liquid, and a salt } \\
\text { crystal layer was forming. The } \\
\text { reel tape was operating properly } \\
\text { and was contacting the salt cake } \\
\text { just below the liquid surface. }\end{array}$ \\
\hline $\mathrm{F}$ & 25 & B-02 & (I) & 05/15/02 & CCTV & I & 825 & $\begin{array}{l}\text { CCTV was used to facilitate the } \\
\text { flushing of the HLLCP and reel } \\
\text { tape. The HLLCP was free of } \\
\text { obstructions and was not } \\
\text { contacting the waste. The reel } \\
\text { tape was free of obstructions } \\
\text { and was operating properly. }\end{array}$ \\
\hline $\mathrm{F}$ & 25 & B-02 & (I) & $12 / 16 / 02$ & CCTV & l & 825 & $\begin{array}{l}\text { CCTV was used to deploy a new } \\
\text { HLLCP. The HLLCP was } \\
\text { properly deployed. }\end{array}$ \\
\hline $\mathrm{F}$ & 25 & B-05 & (I) & $06 / 21 / 02$ & CCTV & l & 825 & $\begin{array}{l}\text { CCTV was used to leak check } \\
\text { the downcomer. No leaks were } \\
\text { observed. }\end{array}$ \\
\hline
\end{tabular}




\begin{tabular}{|c|c|c|c|c|c|c|c|c|}
\hline \multirow{2}{*}{$\frac{\text { AREA }}{F}$} & \multirow{2}{*}{$\begin{array}{c}\text { TANK OR } \\
\text { ANCILLARY } \\
25\end{array}$} & \multicolumn{2}{|c|}{$\frac{\text { ACCESS OPENING }}{(\text { A OR I) }}$} & \multirow{2}{*}{$\frac{\text { DATE }}{06 / 22 / 02}$} & \multicolumn{3}{|c|}{$\begin{array}{l}\text { INSPECTION METHOD } \\
\text { IDENTIFICATION NUMBER }\end{array}$} & \multirow{2}{*}{$\begin{array}{l}\text { REMARKS } \\
\text { CCTV was used to leak check } \\
\text { the downcomer. No leaks were } \\
\text { observed. }\end{array}$} \\
\hline & & B-05 & (I) & & CCTV & l & 825 & \\
\hline $\mathrm{F}$ & 26 & A-01 & (A) & $01 / 29 / 02$ & WAP & l & P02041:04 & Tank condition was normal. \\
\hline $\mathrm{F}$ & 26 & A-02 & (A) & $01 / 29 / 02$ & WAP & l & P02041:13 & Tank condition was normal. \\
\hline $\mathrm{F}$ & 26 & A-02 & (A) & $01 / 31 / 02$ & CCTV & l & $808 \mathrm{~A}$ & $\begin{array}{l}\text { The conductivity probe was } \\
\text { deployed within the setpoint } \\
\text { limits. }\end{array}$ \\
\hline $\mathrm{F}$ & 26 & A-03 & (A) & $01 / 29 / 02$ & WAP & l & P02041:09 & Tank condition was normal. \\
\hline $\mathrm{F}$ & 26 & A-03 & (A) & $01 / 31 / 02$ & CCTV & l & $808 \mathrm{~A}$ & $\begin{array}{l}\text { The conductivity probe was } \\
\text { deployed within the setpoint } \\
\text { limits. }\end{array}$ \\
\hline $\mathrm{F}$ & 26 & A-04 & (A) & $01 / 29 / 02$ & WAP & l & P02041:06 & Tank condition was normal. \\
\hline $\mathrm{F}$ & 26 & A-04 & (A) & $01 / 31 / 02$ & CCTV & l & $808 \mathrm{~A}$ & $\begin{array}{l}\text { The conductivity probe was } \\
\text { deployed within the setpoint } \\
\text { limits. }\end{array}$ \\
\hline $\mathrm{F}$ & 26 & P-01 & (A) & $01 / 29 / 02$ & WAP & I & P02041:05 & Tank condition was normal. \\
\hline $\mathrm{F}$ & 26 & P-02 & (A) & $01 / 29 / 02$ & WAP & l & P02041:03 & Tank condition was normal. \\
\hline $\mathrm{F}$ & 26 & P-03 & (A) & $01 / 29 / 02$ & WAP & l & P02041:02 & Tank condition was normal. \\
\hline $\mathrm{F}$ & 26 & P-04 & (A) & $01 / 29 / 02$ & WAP & l & P02041:01 & Tank condition was normal. \\
\hline $\mathrm{F}$ & 26 & P-05 & (A) & $01 / 31 / 02$ & $\mathrm{DP}$ & l & P02045:01-25 & Tank condition was normal. \\
\hline $\mathrm{F}$ & 26 & P-06 & (A) & $01 / 29 / 02$ & WAP & / & P02041:14 & Tank condition was normal. \\
\hline $\mathrm{F}$ & 26 & P-06 & (A) & $01 / 31 / 02$ & $\mathrm{DP}$ & l & P02046:01-25 & Tank condition was normal. \\
\hline $\mathrm{F}$ & 26 & P-07 & (A) & 01/29/02 & WAP & l & P02041:12 & Tank condition was normal. \\
\hline $\mathrm{F}$ & 26 & P-08 & (A) & $01 / 29 / 02$ & WAP & l & P02041:11 & Tank condition was normal. \\
\hline $\mathrm{F}$ & 26 & P-09 & (A) & $01 / 29 / 02$ & WAP & l & P02041:10 & Tank condition was normal. \\
\hline $\mathrm{F}$ & 26 & $\mathrm{P}-10$ & (A) & 01/29/02 & WAP & I & P02041:08 & Tank condition was normal. \\
\hline $\mathrm{F}$ & 26 & $\mathrm{P}-11$ & (A) & 01/29/02 & WAP & / & P02041:07 & Tank condition was normal. \\
\hline $\mathrm{F}$ & 26 & $\mathrm{P}-12$ & (A) & $01 / 31 / 02$ & DP & l & P02047:01-26 & Tank condition was normal. \\
\hline $\mathrm{F}$ & 26 & $P-13$ & (A) & 02/18/02 & $\mathrm{DP}$ & l & P02048:01-27 & Tank condition was normal. \\
\hline
\end{tabular}




\begin{tabular}{|c|c|c|c|c|c|c|c|c|}
\hline \multirow{2}{*}{$\frac{\text { AREA }}{F}$} & \multirow{2}{*}{$\begin{array}{c}\begin{array}{c}\text { TANK OR } \\
\text { ANCILLARY }\end{array} \\
26\end{array}$} & \multicolumn{2}{|c|}{$\frac{\text { ACCESS OPENING }}{(\text { A OR I) }}$} & \multirow{2}{*}{$\frac{\text { DATE }}{02 / 18 / 02}$} & \multicolumn{3}{|c|}{$\begin{array}{l}\text { INSPECTION METHOD } \\
\text { IDENTIFICATION NUMBER }\end{array}$} & \multirow{2}{*}{$\frac{\text { REMARKS }}{\text { Tank condition was normal. }}$} \\
\hline & & P-14 & (A) & & DP & / & P02049:01-26 & \\
\hline $\mathrm{F}$ & 27 & $\mathrm{~A}-01$ & (A) & 01/29/02 & WAP & / & P02043:10 & Tank condition was normal. \\
\hline $\mathrm{F}$ & 27 & A-02 & (A) & 01/29/02 & WAP & I & P02043:05 & Tank condition was normal. \\
\hline $\mathrm{F}$ & 27 & $\mathrm{~A}-02$ & (A) & 01/31/02 & CCTV & l & $808 \mathrm{~A}$ & $\begin{array}{l}\text { The conductivity probe was } \\
\text { deployed within the setpoint } \\
\text { limits. }\end{array}$ \\
\hline $\mathrm{F}$ & 27 & A-03 & (A) & 01/29/02 & WAP & / & P02043:01 & Tank condition was normal. \\
\hline $\mathrm{F}$ & 27 & A-03 & (A) & 01/31/02 & CCTV & / & $808 \mathrm{~A}$ & $\begin{array}{l}\text { The conductivity probe was } \\
\text { deployed within the setpoint } \\
\text { limits. }\end{array}$ \\
\hline $\mathrm{F}$ & 27 & A-04 & (A) & 01/29/02 & WAP & / & P02043:11 & Tank condition was normal. \\
\hline $\mathrm{F}$ & 27 & A-04 & (A) & $01 / 31 / 02$ & CCTV & l & $808 \mathrm{~A}$ & $\begin{array}{l}\text { The conductivity probe was } \\
\text { deployed within the setpoint } \\
\text { limits. }\end{array}$ \\
\hline $\mathrm{F}$ & 27 & P-01 & (A) & 01/29/02 & WAP & I & P02043:09 & Tank condition was normal. \\
\hline $\mathrm{F}$ & 27 & P-02 & (A) & 01/29/02 & WAP & I & P02043:08 & Tank condition was normal. \\
\hline $\mathrm{F}$ & 27 & P-03 & (A) & 01/29/02 & WAP & / & P02043:07 & Tank condition was normal. \\
\hline $\mathrm{F}$ & 27 & P-04 & (A) & 01/29/02 & WAP & I & P02043:06 & Tank condition was normal. \\
\hline $\mathrm{F}$ & 27 & P-05 & (A) & 01/23/02 & DP & l & P02014:01-27 & Tank condition was normal. \\
\hline $\mathrm{F}$ & 27 & P-06 & (A) & 01/23/02 & DP & I & P02015:01-28 & Tank condition was normal. \\
\hline $\mathrm{F}$ & 27 & P-07 & (A) & 01/29/02 & WAP & / & P02043:04 & Tank condition was normal. \\
\hline $\mathrm{F}$ & 27 & P-08 & (A) & 01/29/02 & WAP & I & P02043:03 & Tank condition was normal. \\
\hline $\mathrm{F}$ & 27 & P-09 & (A) & 01/29/02 & WAP & / & P02043:02 & Tank condition was normal. \\
\hline $\mathrm{F}$ & 27 & $P-10$ & (A) & 01/29/02 & WAP & / & P02043:13 & Tank condition was normal. \\
\hline $\mathrm{F}$ & 27 & $P-11$ & (A) & 01/29/02 & WAP & / & P02043:12 & Tank condition was normal. \\
\hline $\mathrm{F}$ & 27 & P-12 & (A) & 01/23/02 & DP & l & P02016:01-27 & Tank condition was normal. \\
\hline $\mathrm{F}$ & 27 & $P-13$ & (A) & 01/23/02 & DP & / & P02017:01-25 & Tank condition was normal. \\
\hline $\mathrm{F}$ & 27 & $P-14$ & (A) & $01 / 23 / 02$ & DP & 1 & P02018:01-25 & Tank condition was normal. \\
\hline
\end{tabular}




\begin{tabular}{|c|c|c|c|c|c|c|c|c|}
\hline \multirow{2}{*}{$\frac{\text { AREA }}{F}$} & \multirow{2}{*}{$\begin{array}{c}\text { TANK OR } \\
\text { ANCILLARY } \\
27\end{array}$} & \multicolumn{2}{|c|}{$\frac{\text { ACCESS OPENING }}{(\text { A OR I) }}$} & \multirow{2}{*}{$\frac{\text { DATE }}{12 / 28 / 02}$} & \multicolumn{3}{|c|}{$\begin{array}{l}\text { INSPECTION METHOD } \\
\text { IDENTIFICATION NUMBER }\end{array}$} & \multirow{2}{*}{$\begin{array}{l}\qquad \frac{\text { REMARKS }}{\text { Inspection verified that the }} \\
\text { HLLCP was properly deployed. }\end{array}$} \\
\hline & & E-02 & (I) & & CCTV & / & 872 & \\
\hline $\mathrm{F}$ & 27 & $\mathrm{H}$ & (I) & 09/23/02 & CCTV & l & 872 & $\begin{array}{l}\text { CCTV was used to investigate } \\
\text { reel tape inconsistencies. } \\
\text { Inspection of the reel tape was } \\
\text { inconclusive. The bottom of the } \\
\text { reel tape "bob" was not visible. } \\
\text { The surface of the waste was } \\
\text { covered with a crystallized layer } \\
\text { of salt which appeared to be } \\
\text { uniform throughout the tank. }\end{array}$ \\
\hline $\mathrm{F}$ & 28 & $\begin{array}{r}\text { Underliner } \\
\text { Sump }\end{array}$ & & 03/27/02 & CCTV & l & 809D & $\begin{array}{l}\text { The conductivity probe could not } \\
\text { be properly deployed due to an } \\
\text { obstruction in the standpipe. }\end{array}$ \\
\hline $\mathrm{F}$ & 28 & $\begin{array}{r}\text { Underliner } \\
\text { Sump }\end{array}$ & & 05/02/02 & CCTV & / & 807A & $\begin{array}{l}\text { Inspection revealed that the } \\
\text { conductivity probe extended } \\
\text { beneath the standpipe. }\end{array}$ \\
\hline $\mathrm{F}$ & 28 & $A-01$ & $(\mathrm{~A})$ & $01 / 29 / 02$ & WAP & l & P02044:03 & Tank condition was normal. \\
\hline $\mathrm{F}$ & 28 & A-02 & (A) & $01 / 29 / 02$ & WAP & l & P02044:10 & Tank condition was normal. \\
\hline $\mathrm{F}$ & 28 & A-02 & (A) & $01 / 31 / 02$ & CCTV & / & $808 \mathrm{~A}$ & $\begin{array}{l}\text { The conductivity probe was } \\
\text { deployed within the setpoint } \\
\text { limits. }\end{array}$ \\
\hline $\mathrm{F}$ & 28 & A-03 & (A) & $01 / 29 / 02$ & WAP & / & P02044:07 & Tank condition was normal. \\
\hline $\mathrm{F}$ & 28 & A-03 & (A) & 01/31/02 & CCTV & l & $808 \mathrm{~A}$ & $\begin{array}{l}\text { The conductivity probe was } \\
\text { deployed within the setpoint } \\
\text { limits. }\end{array}$ \\
\hline $\mathrm{F}$ & 28 & A-04 & (A) & $01 / 29 / 02$ & WAP & / & P02044:05 & Tank condition was normal. \\
\hline $\mathrm{F}$ & 28 & A-04 & (A) & $01 / 31 / 02$ & CCTV & / & $808 \mathrm{~A}$ & $\begin{array}{l}\text { The conductivity probe was } \\
\text { deployed within the setpoint } \\
\text { limits. }\end{array}$ \\
\hline $\mathrm{F}$ & 28 & P-01 & (A) & $01 / 29 / 02$ & WAP & 1 & P02044:02 & Tank condition was normal. \\
\hline $\mathrm{F}$ & 28 & P-02 & (A) & 01/23/02 & DP & 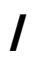 & P02019:01-25 & Tank condition was normal. \\
\hline $\mathrm{F}$ & 28 & P-03 & (A) & $01 / 29 / 02$ & WAP & 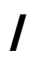 & P02044:01 & Tank condition was normal. \\
\hline $\mathrm{F}$ & 28 & P-04 & (A) & $01 / 29 / 02$ & WAP & & P02044:12 & Tank condition was normal. \\
\hline $\mathrm{F}$ & 28 & P-05 & (A) & $01 / 23 / 02$ & DP & & P02020:01-25 & Tank condition was normal. \\
\hline $\mathrm{F}$ & 28 & P-06 & $(\mathrm{A})$ & $01 / 29 / 02$ & WAP & l & P02044:11 & Tank condition was normal. \\
\hline
\end{tabular}




\begin{tabular}{|c|c|c|c|c|c|c|c|c|}
\hline \multirow{2}{*}{$\frac{\text { AREA }}{F}$} & \multirow{2}{*}{$\begin{array}{c}\text { TANK OR } \\
\text { ANCILLARY } \\
28\end{array}$} & \multicolumn{2}{|c|}{$\frac{\text { ACCESS OPENING }}{(\text { A OR I) }}$} & \multirow{2}{*}{$\frac{\text { DATE }}{01 / 29 / 02}$} & \multicolumn{3}{|c|}{$\begin{array}{l}\text { INSPECTION METHOD } \\
\text { IDENTIFICATION NUMBER }\end{array}$} & \multirow{2}{*}{$\frac{\text { REMARKS }}{\text { Tank condition was normal. }}$} \\
\hline & & P-07 & (A) & & WAP & / & P02044:09 & \\
\hline $\mathrm{F}$ & 28 & P-08 & (A) & $01 / 29 / 02$ & WAP & / & P02044:08 & Tank condition was normal. \\
\hline $\mathrm{F}$ & 28 & P-09 & (A) & $01 / 23 / 02$ & $\mathrm{DP}$ & l & P02021:01-25 & Tank condition was normal. \\
\hline $\mathrm{F}$ & 28 & $P-10$ & (A) & $01 / 23 / 02$ & $\mathrm{DP}$ & l & P02022:01-26 & Tank condition was normal. \\
\hline $\mathrm{F}$ & 28 & $P-11$ & (A) & $01 / 29 / 02$ & WAP & / & P02044:06 & Tank condition was normal. \\
\hline $\mathrm{F}$ & 28 & $\mathrm{P}-12$ & (A) & $01 / 29 / 02$ & WAP & / & P02044:04 & Tank condition was normal. \\
\hline $\mathrm{F}$ & 28 & $P-13$ & (A) & $01 / 23 / 02$ & $\mathrm{DP}$ & / & P02023:01-25 & Tank condition was normal. \\
\hline $\mathrm{F}$ & 28 & $\mathrm{P}-14$ & (A) & $01 / 23 / 02$ & $\mathrm{DP}$ & l & P02024:01-25 & Tank condition was normal. \\
\hline $\mathrm{H}$ & 29 & LPS & & 08/28/02 & CCTV & / & $809 \mathrm{H}$ & $\begin{array}{l}\text { CCTV was used to document the } \\
\text { conditions of the LPS. Deposits } \\
\text { were observed at the bottom of } \\
\text { the LPS. }\end{array}$ \\
\hline $\mathrm{H}$ & 29 & $A-01$ & (A) & 01/18/02 & WAP & l & P02004:07 & Tank condition was normal. \\
\hline $\mathrm{H}$ & 29 & $A-01$ & (A) & $01 / 26 / 02$ & CCTV & / & 808 & $\begin{array}{l}\text { The conductivity probe was } \\
\text { deployed within the setpoint } \\
\text { limits. }\end{array}$ \\
\hline $\mathrm{H}$ & 29 & A-02 & (A) & 01/18/02 & WAP & l & P02004:06 & Tank condition was normal. \\
\hline $\mathrm{H}$ & 29 & A-02 & (A) & $01 / 26 / 02$ & CCTV & l & 808 & $\begin{array}{l}\text { The conductivity probe was } \\
\text { deployed within the setpoint } \\
\text { limits. }\end{array}$ \\
\hline $\mathrm{H}$ & 29 & A-03 & $(A)$ & 01/18/02 & WAP & / & P02004:12 & Tank condition was normal. \\
\hline $\mathrm{H}$ & 29 & A-03 & $(\mathrm{A})$ & $01 / 26 / 02$ & CCTV & l & 808 & $\begin{array}{l}\text { The conductivity probe was } \\
\text { deployed within the setpoint } \\
\text { limits. }\end{array}$ \\
\hline $\mathrm{H}$ & 29 & A-04 & (A) & 01/18/02 & WAP & / & P02004:03 & Tank condition was normal. \\
\hline $\mathrm{H}$ & 29 & A-04 & (A) & 01/26/02 & CCTV & / & 808 & $\begin{array}{l}\text { The magnetically mounted } \\
\text { thermocouple was deployed } \\
\text { within the setpoint limits. }\end{array}$ \\
\hline $\mathrm{H}$ & 29 & P-01 & (A) & 01/18/02 & WAP & l & P02004:01 & Tank condition was normal. \\
\hline $\mathrm{H}$ & 29 & P-02 & (A) & 01/18/02 & WAP & / & P02004:02 & Tank condition was normal. \\
\hline $\mathrm{H}$ & 29 & P-03 & (A) & $01 / 25 / 02$ & $\mathrm{DP}$ & / & P02050:01-25 & Tank condition was normal. \\
\hline
\end{tabular}




\begin{tabular}{|c|c|c|c|c|c|c|c|c|}
\hline \multirow{2}{*}{ AREA } & \multirow{2}{*}{$\begin{array}{c}\text { TANK OR } \\
\text { ANCILLARY } \\
29\end{array}$} & \multicolumn{2}{|c|}{$\frac{\text { ACCESS OPENING }}{(\text { A OR I) }}$} & \multirow{2}{*}{$\frac{\text { DATE }}{01 / 18 / 02}$} & \multicolumn{3}{|c|}{$\begin{array}{l}\text { INSPECTION METHOD } \\
\text { IDENTIFICATION NUMBER }\end{array}$} & \multirow{2}{*}{$\frac{\text { REMARKS }}{\text { Tank condition was normal. }}$} \\
\hline & & P-04 & (A) & & WAP & / & P02004:04 & \\
\hline $\mathrm{H}$ & 29 & P-05 & (A) & 01/18/02 & WAP & / & P02004:05 & Tank condition was normal. \\
\hline $\mathrm{H}$ & 29 & P-06 & (A) & 01/25/02 & DP & / & P02051:01-25 & Tank condition was normal. \\
\hline $\mathrm{H}$ & 29 & P-07 & (A) & 01/18/02 & WAP & / & P02004:08 & Tank condition was normal. \\
\hline $\mathrm{H}$ & 29 & P-08 & (A) & 01/18/02 & WAP & l & P02004:09 & Tank condition was normal. \\
\hline $\mathrm{H}$ & 29 & P-09 & (A) & $01 / 25 / 02$ & DP & / & P02052:01-25 & Tank condition was normal. \\
\hline $\mathrm{H}$ & 29 & $P-10$ & (A) & 01/25/02 & DP & / & P02053:01-25 & Tank condition was normal. \\
\hline $\mathrm{H}$ & 29 & $P-11$ & (A) & 01/18/02 & WAP & / & P02004:10 & Tank condition was normal. \\
\hline $\mathrm{H}$ & 29 & P-12 & (A) & 01/18/02 & WAP & / & P02004:11 & Tank condition was normal. \\
\hline $\mathrm{H}$ & 29 & $P-13$ & (A) & $01 / 25 / 02$ & DP & / & P02054:01-27 & Tank condition was normal. \\
\hline $\mathrm{H}$ & 29 & $P-14$ & (A) & 01/18/02 & WAP & l & P02004:13 & Tank condition was normal. \\
\hline $\mathrm{H}$ & 30 & $A-01$ & (A) & 01/19/02 & WAP & / & P02007:11 & $\begin{array}{l}\text { Tank condition was normal. } \\
\text { Stains observed on the } \\
\text { secondary vessel wall were due } \\
\text { to the inleakage of water. }\end{array}$ \\
\hline $\mathrm{H}$ & 30 & $A-01$ & (A) & 01/29/02 & CCTV & / & $808 \mathrm{~A}$ & $\begin{array}{l}\text { The conductivity probe was } \\
\text { deployed within the setpoint } \\
\text { limits. }\end{array}$ \\
\hline $\mathrm{H}$ & 30 & A-02 & (A) & 01/29/02 & CCTV & / & $808 \mathrm{~A}$ & $\begin{array}{l}\text { The conductivity probe was } \\
\text { deployed within the setpoint } \\
\text { limits. }\end{array}$ \\
\hline $\mathrm{H}$ & 30 & A-02 & (A) & 05/05/02 & WAP & / & P02007:13 & Tank condition was normal. \\
\hline $\mathrm{H}$ & 30 & A-03 & (A) & 01/19/02 & WAP & / & P02007:09 & Tank condition was normal. \\
\hline $\mathrm{H}$ & 30 & A-03 & (A) & 01/29/02 & CCTV & / & $808 \mathrm{~A}$ & $\begin{array}{l}\text { The conductivity probe was } \\
\text { deployed within the setpoint } \\
\text { limits. }\end{array}$ \\
\hline $\mathrm{H}$ & 30 & A-04 & (A) & 01/19/02 & WAP & / & P02007:06 & $\begin{array}{l}\text { Tank condition was normal. } \\
\text { Stains observed on the } \\
\text { secondary vessel wall were due } \\
\text { to the inleakage of water. }\end{array}$ \\
\hline $\mathrm{H}$ & 30 & $A-04$ & (A) & 01/29/02 & CCTV & / & $808 \mathrm{~A}$ & $\begin{array}{l}\text { The magnetically mounted } \\
\text { thermocouple was deployed } \\
\text { within the setpoint limits. }\end{array}$ \\
\hline
\end{tabular}




\begin{tabular}{|c|c|c|c|c|c|c|c|c|}
\hline \multirow{2}{*}{$\frac{\text { AREA }}{\mathrm{H}}$} & \multirow{2}{*}{$\begin{array}{c}\text { TANK OR } \\
\text { ANCILLARY } \\
30\end{array}$} & \multicolumn{2}{|c|}{$\frac{\text { ACCESS OPENING }}{(\text { A OR I) }}$} & \multirow{2}{*}{$\frac{\text { DATE }}{01 / 19 / 02}$} & \multicolumn{3}{|c|}{$\begin{array}{l}\text { INSPECTION METHOD } \\
\text { IDENTIFICATION NUMBER }\end{array}$} & \multirow{2}{*}{$\frac{\text { REMARKS }}{\text { Tank condition was normal. }}$} \\
\hline & & P-01 & (A) & & WAP & l & P02007:02 & \\
\hline $\mathrm{H}$ & 30 & P-02 & (A) & 01/19/02 & WAP & / & P02007:01 & $\begin{array}{l}\text { Tank condition was normal. } \\
\text { Stains observed on the } \\
\text { secondary vessel wall were due } \\
\text { to the inleakage of water. }\end{array}$ \\
\hline $\mathrm{H}$ & 30 & P-03 & $(\mathrm{A})$ & $01 / 24 / 02$ & DP & l & P02067:01-26 & Tank condition was normal. \\
\hline $\mathrm{H}$ & 30 & P-04 & (A) & $01 / 19 / 02$ & WAP & l & P02007:05 & Tank condition was normal. \\
\hline $\mathrm{H}$ & 30 & P-05 & (A) & $01 / 19 / 02$ & WAP & / & P02007:12 & Tank condition was normal. \\
\hline $\mathrm{H}$ & 30 & P-06 & (A) & $01 / 24 / 02$ & DP & / & P02068:01-27 & Tank condition was normal. \\
\hline $\mathrm{H}$ & 30 & P-07 & (A) & 01/19/02 & WAP & I & P02007:04 & Tank condition was normal. \\
\hline $\mathrm{H}$ & 30 & P-08 & (A) & 01/19/02 & WAP & l & P02007:10 & $\begin{array}{l}\text { Tank condition was normal. } \\
\text { Stains observed on the } \\
\text { secondary vessel wall were due } \\
\text { to the inleakage of water. }\end{array}$ \\
\hline $\mathrm{H}$ & 30 & P-09 & (A) & $01 / 24 / 02$ & DP & I & P02069:01-26 & Tank condition was normal. \\
\hline $\mathrm{H}$ & 30 & $P-10$ & $(\mathrm{~A})$ & $01 / 24 / 02$ & DP & l & P02070:01-27 & Tank condition was normal. \\
\hline $\mathrm{H}$ & 30 & $P-11$ & (A) & 01/19/02 & WAP & / & P02007:07 & Tank condition was normal. \\
\hline $\mathrm{H}$ & 30 & $\mathrm{P}-12$ & (A) & 01/19/02 & WAP & l & P02007:08 & Tank condition was normal. \\
\hline $\mathrm{H}$ & 30 & $P-13$ & $(\mathrm{~A})$ & $01 / 24 / 02$ & DP & l & P02071:01-27 & $\begin{array}{l}\text { Tank condition was normal. } \\
\text { Stains and marks on the primary } \\
\text { vessel wall had increased since } \\
\text { last inspected. A masselin cloth } \\
\text { was observed on the annulus } \\
\text { floor. }\end{array}$ \\
\hline $\mathrm{H}$ & 30 & $\mathrm{P}-14$ & $(\mathrm{~A})$ & $01 / 19 / 02$ & WAP & / & P02007:03 & Tank condition was normal. \\
\hline $\mathrm{H}$ & 30 & B-01 & (l) & 07/11/02 & CCTV & / & 844 & $\begin{array}{l}\text { Inspection revealed } \\
\text { approximately } 7 \text { inches of salt } \\
\text { accumulation on the cooling coil } \\
\text { bundles. }\end{array}$ \\
\hline $\mathrm{H}$ & 30 & B-01 & (l) & 07/17/02 & CCTV & / & 850 & $\begin{array}{l}\text { Inspection revealed } \\
\text { approximately } 8 \text { inches of salt } \\
\text { accumulation on the B2 and B10 } \\
\text { cooling coil bundles. }\end{array}$ \\
\hline $\mathrm{H}$ & 30 & B-01 & (I) & $07 / 24 / 02$ & CCTV & l & 850 & $\begin{array}{l}\text { Inspection revealed } \\
\text { approximately } 8 \text { inches of salt } \\
\text { accumulation on the cooling coil } \\
\text { bundles. }\end{array}$ \\
\hline
\end{tabular}




\begin{tabular}{|c|c|c|c|c|c|c|c|c|}
\hline \multirow{2}{*}{$\frac{\text { AREA }}{\mathrm{H}}$} & \multirow{2}{*}{$\begin{array}{c}\text { TANK OR } \\
\text { ANCILLARY } \\
30\end{array}$} & \multicolumn{2}{|c|}{$\frac{\text { ACCESS OPENING }}{(\text { A OR I) }}$} & \multirow{2}{*}{$\frac{\text { DATE }}{03 / 14 / 02}$} & \multicolumn{3}{|c|}{$\begin{array}{l}\text { INSPECTION METHOD } \\
\text { IDENTIFICATION NUMBER }\end{array}$} & \multirow{2}{*}{$\begin{array}{l}\qquad \text { REMARKS } \\
\text { CCTV verified positive flow } \\
\text { through the backflush valve as } \\
\text { the gravity drain line was } \\
\text { pressure washed from COP } 103 .\end{array}$} \\
\hline & & C-01 & (I) & & CCTV & I & $809 C$ & \\
\hline $\mathrm{H}$ & 30 & C-01 & (I) & 05/15/02 & CCTV & I & 827 & $\begin{array}{l}\text { CCTV was used to monitor for } \\
\text { salt accumulation on the cooling } \\
\text { coil bundles. No excessive salt } \\
\text { loading was observed on the } \\
\text { bundles. }\end{array}$ \\
\hline $\mathrm{H}$ & 30 & C-01 & (I) & 09/06/02 & CCTV & I & 850 & $\begin{array}{l}\text { CCTV was used to leak check } \\
\text { the TTJ. Liquid was observed } \\
\text { spraying from the TTJ. }\end{array}$ \\
\hline $\mathrm{H}$ & 30 & C-02 & (I) & 03/13/02 & CCTV & I & $809 \mathrm{C}$ & $\begin{array}{l}\text { Inspection of the backflush valve } \\
\text { via the catheter line revealed no } \\
\text { obstructions. Some loose solids } \\
\text { were observed on the bottom of } \\
\text { the GDL. }\end{array}$ \\
\hline $\mathrm{H}$ & 30 & $\mathrm{H}$ & (I) & $01 / 16 / 02$ & CCTV & I & $809 A$ & $\begin{array}{l}\text { Inspection revealed that the } \\
\text { cooling coil bundles were } \\
\text { covered with salt ranging from a } \\
\text { thin coating near the top to a few } \\
\text { inches near the surface. A salt } \\
\text { crust layer had formed on the } \\
\text { waste surface near the H riser. } \\
\text { Liquid was visible through the } \\
\text { cracks in the salt crust. Other } \\
\text { areas of the tank appeared to } \\
\text { have a liquid surface. A salt } \\
\text { coating had formed on the wall } \\
\text { with areas near the waste } \\
\text { surface approximately one inch } \\
\text { thick. A thin layer of salt had } \\
\text { formed on the center column. }\end{array}$ \\
\hline $\mathrm{H}$ & 30 & $\mathrm{H}$ & (I) & 05/27/02 & CCTV & I & 836 & $\begin{array}{l}\text { CCTV was used to leak check } \\
\text { the transfer jet and to document } \\
\text { salt accumulation on the cooling } \\
\text { coil bundles. No leaks were } \\
\text { observed. Approximately } 6 \\
\text { inches of salt accumulation were } \\
\text { observed on the coil bundles } \\
\text { above the waste level. }\end{array}$ \\
\hline $\mathrm{H}$ & 30 & $\mathrm{H}$ & (I) & 06/03/02 & CCTV & I & 838 & $\begin{array}{l}\text { CCTV was used to document } \\
\text { salt accumulation on the cooling } \\
\text { coil bundles during transfer. } \\
\text { Approximately } 8 \text { to } 10 \text { inches of } \\
\text { salt crust were observed above } \\
\text { the waste level. }\end{array}$ \\
\hline
\end{tabular}




\section{TANK OR ANCILLARY \\ AREA}

$\mathrm{H}$

30

$\frac{\text { ACCESS OPENING }}{\text { (A OR I) }}$

DATE

$\mathrm{H}$ (I)

06/04/02

H (I)

$08 / 27 / 02$

CCTV /

850

$\mathrm{H}$

30

$\mathrm{H}$

30

$\mathrm{H}$

$\mathrm{H}$

$\mathrm{H}$

30

$\mathrm{H}$

30

H (I)

09/08/02

CCTV

I

846

$\mathrm{H}$

30

H (I)

H (I)

H (I)

H (I)

09/07/02

CCTV

864

864

CCTV I

864 


\begin{tabular}{|c|c|c|c|c|c|c|c|c|}
\hline \multirow{2}{*}{$\frac{\text { AREA }}{\mathrm{H}}$} & \multirow{2}{*}{$\begin{array}{c}\begin{array}{c}\text { TANK OR } \\
\text { ANCILLARY }\end{array} \\
30\end{array}$} & \multicolumn{2}{|c|}{$\frac{\text { ACCESS OPENING }}{\text { (A OR I) }}$} & \multirow{2}{*}{$\frac{\text { DATE }}{10 / 23 / 02}$} & \multicolumn{3}{|c|}{$\begin{array}{l}\text { INSPECTION METHOD } \\
\text { IDENTIFICATION NUMBER }\end{array}$} & \multirow{2}{*}{$\begin{array}{l}\text { REMARKS } \\
\text { Inspection was made to } \\
\text { document salt accumulation on } \\
\text { the cooling coil bundles. The } \\
\text { bundles were encrusted with salt } \\
\text { approximately } 3 \text { inches thick. }\end{array}$} \\
\hline & & $\mathrm{H}$ & (I) & & CCTV & l & 864 & \\
\hline $\mathrm{H}$ & 30 & $\mathrm{H}$ & (I) & $10 / 30 / 02$ & CCTV & l & 864 & $\begin{array}{l}\text { Inspection was made to } \\
\text { document salt accumulation on } \\
\text { the cooling coil bundles. No salt } \\
\text { was observed above the liquid } \\
\text { level. }\end{array}$ \\
\hline $\mathrm{H}$ & 30 & $\mathrm{H}$ & (l) & $10 / 30 / 02$ & CCTV & l & 864 & $\begin{array}{l}\text { Inspection was made to } \\
\text { document salt accumulation on } \\
\text { the cooling coil bundles. No salt } \\
\text { was observed above the liquid } \\
\text { level. }\end{array}$ \\
\hline $\mathrm{H}$ & 31 & $A-01$ & $(\mathrm{~A})$ & 01/18/02 & WAP & l & P02005:06 & Tank condition was normal. \\
\hline $\mathrm{H}$ & 31 & A-01 & (A) & $01 / 28 / 02$ & CCTV & l & 808 & $\begin{array}{l}\text { The conductivity probe was } \\
\text { deployed within the setpoint } \\
\text { limits. }\end{array}$ \\
\hline $\mathrm{H}$ & 31 & A-02 & (A) & $01 / 28 / 02$ & CCTV & l & 808 & $\begin{array}{l}\text { The conductivity probe was } \\
\text { deployed within the setpoint } \\
\text { limits. }\end{array}$ \\
\hline $\mathrm{H}$ & 31 & A-02 & (A) & 05/05/02 & WAP & l & P02005:09 & Tank condition was normal. \\
\hline $\mathrm{H}$ & 31 & A-03 & (A) & $01 / 18 / 02$ & WAP & l & P02005:13 & Tank condition was normal. \\
\hline $\mathrm{H}$ & 31 & A-03 & (A) & $01 / 28 / 02$ & CCTV & l & 808 & $\begin{array}{l}\text { The conductivity probe was } \\
\text { deployed within the setpoint } \\
\text { limits. }\end{array}$ \\
\hline $\mathrm{H}$ & 31 & A-04 & $(\mathrm{A})$ & $01 / 18 / 02$ & WAP & / & P02005:03 & Tank condition was normal. \\
\hline $\mathrm{H}$ & 31 & A-04 & (A) & $01 / 28 / 02$ & CCTV & I & 808 & $\begin{array}{l}\text { The magnetically mounted } \\
\text { thermocouple was deployed } \\
\text { within the setpoint limits. }\end{array}$ \\
\hline $\mathrm{H}$ & 31 & P-01 & (A) & $01 / 18 / 02$ & WAP & / & P02005:01 & Tank condition was normal. \\
\hline $\mathrm{H}$ & 31 & P-02 & (A) & $01 / 18 / 02$ & WAP & l & P02005:02 & Tank condition was normal. \\
\hline $\mathrm{H}$ & 31 & P-03 & (A) & $01 / 25 / 02$ & $\mathrm{DP}$ & / & P02059:01-27 & Tank condition was normal. \\
\hline $\mathrm{H}$ & 31 & P-04 & (A) & 01/18/02 & WAP & l & P02005:04 & Tank condition was normal. \\
\hline $\mathrm{H}$ & 31 & P-05 & (A) & 01/18/02 & WAP & / & P02005:05 & Tank condition was normal. \\
\hline $\mathrm{H}$ & 31 & P-06 & (A) & $01 / 25 / 02$ & $\mathrm{DP}$ & l & P02060:01-26 & Tank condition was normal. \\
\hline
\end{tabular}




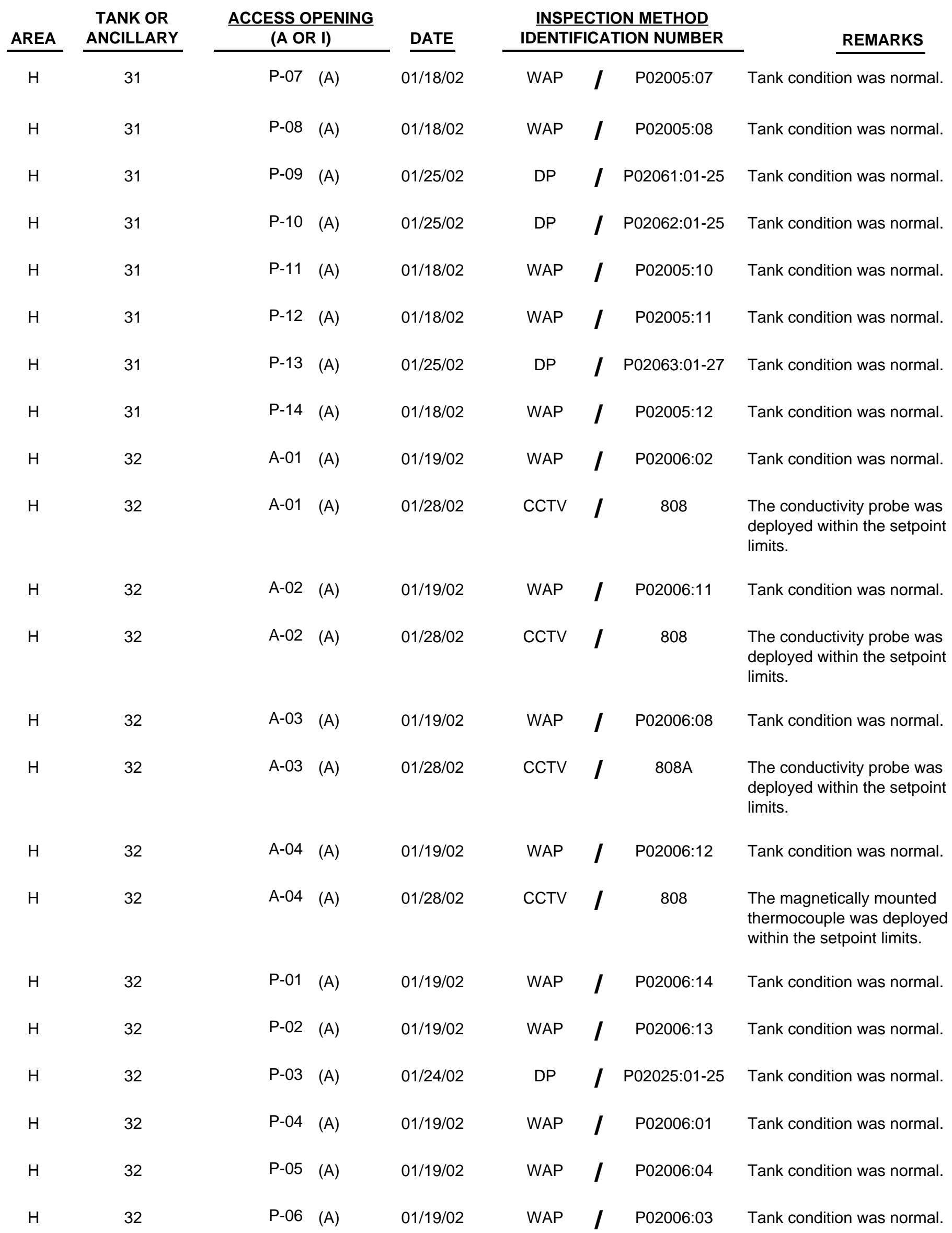




\begin{tabular}{|c|c|c|c|c|c|c|c|c|}
\hline \multirow{2}{*}{$\frac{\text { AREA }}{\mathrm{H}}$} & \multirow{2}{*}{$\begin{array}{c}\begin{array}{c}\text { TANK OR } \\
\text { ANCILLARY }\end{array} \\
32\end{array}$} & \multicolumn{2}{|c|}{$\frac{\text { ACCESS OPENING }}{(\text { A OR I) }}$} & \multirow{2}{*}{$\frac{\text { DATE }}{01 / 24 / 02}$} & \multicolumn{3}{|c|}{$\begin{array}{l}\text { INSPECTION METHOD } \\
\text { IDENTIFICATION NUMBER }\end{array}$} & \multirow{2}{*}{$\begin{array}{l}\text { REMARKS } \\
\text { Tank condition was normal. } \\
\text { Chromate stains on the primary } \\
\text { and secondary vessel wall had } \\
\text { increased since last inspected. }\end{array}$} \\
\hline & & P-06 & (A) & & DP & / & P02028:01-26 & \\
\hline $\mathrm{H}$ & 32 & P-07 & (A) & 01/19/02 & WAP & l & P02006:09 & Tank condition was normal. \\
\hline $\mathrm{H}$ & 32 & P-08 & (A) & 01/19/02 & WAP & l & P02006:10 & Tank condition was normal. \\
\hline $\mathrm{H}$ & 32 & P-09 & (A) & 05/05/02 & DP & / & P02026:01-27 & Tank condition was normal. \\
\hline $\mathrm{H}$ & 32 & $P-10$ & $(\mathrm{~A})$ & $01 / 24 / 02$ & DP & I & P02027:01-25 & $\begin{array}{l}\text { Tank condition was normal. } \\
\text { Stains on the secondary vessel } \\
\text { wall, annulus floor and the top of } \\
\text { the ventilation duct had } \\
\text { increased since last inspected. }\end{array}$ \\
\hline $\mathrm{H}$ & 32 & $\mathrm{P}-11$ & (A) & $01 / 19 / 02$ & WAP & / & P02006:05 & Tank condition was normal. \\
\hline $\mathrm{H}$ & 32 & P-12 & (A) & 01/29/02 & WAP & l & P02085:01 & Tank condition was normal. \\
\hline $\mathrm{H}$ & 32 & $P-13$ & (A) & $01 / 24 / 02$ & DP & l & P02072:01-27 & Tank condition was normal. \\
\hline $\mathrm{H}$ & 32 & P-14 & (A) & 01/19/02 & WAP & / & P02006:06 & Tank condition was normal. \\
\hline $\mathrm{H}$ & 32 & P-15 & (A) & 01/19/02 & WAP & / & P02006:07 & Tank condition was normal. \\
\hline $\mathrm{H}$ & 32 & B-02 & (I) & 07/03/02 & CCTV & I & 847 & $\begin{array}{l}\text { Inspection showed that the } \\
\text { cooling coil bundles were } \\
\text { encrusted in salt from the liquid } \\
\text { level to approximately } 14 \text { inches } \\
\text { above the surface of the liquid. } \\
\text { The salt extended two inches out } \\
\text { from the coils. }\end{array}$ \\
\hline $\mathrm{H}$ & 32 & C-02 & (I) & $10 / 19 / 02$ & CCTV & l & 876 & $\begin{array}{l}\text { CCTV was used to monitor for } \\
\text { leaks at the feed pump. No } \\
\text { leaks were observed. }\end{array}$ \\
\hline $\mathrm{H}$ & 32 & $\mathrm{H}$ & (I) & 09/03/02 & CCTV & I & 847 & $\begin{array}{l}\text { CCTV was used to document } \\
\text { conditions of the transfer jet } \\
\text { before removal. No unusual } \\
\text { conditions were observed. }\end{array}$ \\
\hline $\mathrm{H}$ & 32 & $\mathrm{H}$ & (I) & $10 / 09 / 02$ & CCTV & I & 876 & $\begin{array}{l}\text { Inspection documented the } \\
\text { conditions of the cooling coil } \\
\text { bundles after attempting to } \\
\text { dislodge encrusted salt using a } \\
\text { steam lance inserted into the } \\
\text { tank. The bundles remain } \\
\text { encrusted in salt approximately } \\
6 \text { inches thick extending } 8-9 \text { feet } \\
\text { above the liquid level. }\end{array}$ \\
\hline
\end{tabular}




\begin{tabular}{|c|c|c|c|c|c|c|c|c|}
\hline \multirow{2}{*}{$\frac{\text { AREA }}{\mathrm{H}}$} & \multirow{2}{*}{$\begin{array}{c}\begin{array}{c}\text { TANK OR } \\
\text { ANCILLARY }\end{array} \\
32\end{array}$} & \multicolumn{2}{|c|}{$\frac{\text { ACCESS OPENING }}{(\text { A OR I) }}$} & \multirow{2}{*}{$\frac{\text { DATE }}{10 / 24 / 02}$} & \multicolumn{3}{|c|}{$\begin{array}{l}\text { INSPECTION METHOD } \\
\text { IDENTIFICATION NUMBER }\end{array}$} & \multirow{2}{*}{$\begin{array}{l}\text { REMARKS } \\
\text { CCTV was used to perform I } \\
\text { check of the downcomer. No } \\
\text { leaks were observed. No } \\
\text { accumulation of salt was } \\
\text { observed above the liquid lev }\end{array}$} \\
\hline & & $\mathrm{H}$ & (I) & & CCTV & l & 876 & \\
\hline $\mathrm{F}$ & 33 & A-01 & $(\mathrm{A})$ & 03/29/02 & WAP & l & P02139:01 & Tank condition was normal. \\
\hline $\mathrm{F}$ & 33 & A-01 & (A) & 04/03/02 & CCTV & l & $808 \mathrm{C}$ & $\begin{array}{l}\text { The conductivity probe was } \\
\text { deployed within the setpoint } \\
\text { limits. }\end{array}$ \\
\hline $\mathrm{F}$ & 33 & A-03 & (A) & 03/29/02 & WAP & l & P02139:13 & Tank condition was normal. \\
\hline $\mathrm{F}$ & 33 & A-03 & (A) & 04/09/02 & CCTV & / & $808 \mathrm{C}$ & $\begin{array}{l}\text { The conductivity probe was } \\
\text { deployed within the setpoint } \\
\text { limits. }\end{array}$ \\
\hline $\mathrm{F}$ & 33 & A-04 & (A) & 03/29/02 & WAP & / & P02139:08 & Tank condition was normal. \\
\hline $\mathrm{F}$ & 33 & P-02 & (A) & 03/29/02 & WAP & l & P02139:07 & Tank condition was normal. \\
\hline $\mathrm{F}$ & 33 & P-03 & (A) & $03 / 28 / 02$ & DP & l & P02133:01-27 & Tank condition was normal. \\
\hline $\mathrm{F}$ & 33 & P-04 & (A) & 03/29/02 & WAP & / & P02139:09 & Tank condition was normal. \\
\hline $\mathrm{F}$ & 33 & P-05 & (A) & 03/29/02 & WAP & / & P02139:10 & Tank condition was normal. \\
\hline $\mathrm{F}$ & 33 & P-06 & (A) & 03/28/02 & $\mathrm{DP}$ & / & P02131:01-27 & Tank condition was normal. \\
\hline $\mathrm{F}$ & 33 & P-07 & (A) & 03/29/02 & WAP & l & P02139:02 & Tank condition was normal. \\
\hline $\mathrm{F}$ & 33 & P-08 & $(\mathrm{A})$ & 03/29/02 & WAP & / & P02139:03 & Tank condition was normal. \\
\hline $\mathrm{F}$ & 33 & P-09 & (A) & 03/28/02 & $\mathrm{DP}$ & l & P02132:01-27 & Tank condition was normal. \\
\hline $\mathrm{F}$ & 33 & $P-10$ & $(\mathrm{~A})$ & 03/28/02 & DP & / & P02130:01-27 & Tank condition was normal. \\
\hline $\mathrm{F}$ & 33 & $P-11$ & (A) & 03/29/02 & WAP & l & P02139:05 & Tank condition was normal. \\
\hline
\end{tabular}




\begin{tabular}{|c|c|c|c|c|c|c|c|c|}
\hline \multirow{2}{*}{$\frac{\text { AREA }}{F}$} & \multirow{2}{*}{$\begin{array}{c}\text { TANK OR } \\
\text { ANCILLARY } \\
33\end{array}$} & \multicolumn{2}{|c|}{$\frac{\text { ACCESS OPENING }}{(\text { A OR I) }}$} & \multirow{2}{*}{$\frac{\text { DATE }}{03 / 29 / 02}$} & \multicolumn{3}{|c|}{$\begin{array}{l}\text { INSPECTION METHOD } \\
\text { IDENTIFICATION NUMBER }\end{array}$} & \multirow{2}{*}{$\frac{\text { REMARKS }}{\text { Tank condition was normal. }}$} \\
\hline & & $\mathrm{P}-12$ & (A) & & WAP & l & P02139:11 & \\
\hline $\mathrm{F}$ & 33 & P-13 & (A) & 03/28/02 & DP & / & P02134:01-27 & Tank condition was normal. \\
\hline $\mathrm{F}$ & 33 & $\mathrm{P}-14$ & (A) & 03/29/02 & WAP & / & P02139:12 & Tank condition was normal. \\
\hline $\mathrm{F}$ & 33 & P-15 & (A) & 03/29/02 & WAP & l & P02139:14 & Tank condition was normal. \\
\hline $\mathrm{F}$ & 33 & $\mathrm{P}-16$ & (A) & 03/29/02 & WAP & l & P02139:15 & Tank condition was normal. \\
\hline $\mathrm{F}$ & 33 & E-02 & (I) & 06/11/02 & CCTV & / & $809 \mathrm{H}$ & $\begin{array}{l}\text { CCTV was used to document } \\
\text { conditions beneath the reel tape } \\
\text { and verify proper operation. The } \\
\text { waste surface was liquid, and no } \\
\text { obstructions were observed. } \\
\text { The reel tape was not operating } \\
\text { properly; it would not contact the } \\
\text { liquid. }\end{array}$ \\
\hline $\mathrm{F}$ & 33 & $\mathrm{H}$ & (I) & $11 / 26 / 02$ & CCTV & l & 888 & $\begin{array}{l}\text { Inspection to determine if the } \\
\text { TTJ was leaking was } \\
\text { inconclusive. The TTJ is either } \\
\text { leaking at the stuffing box or } \\
\text { from the anti-siphon break } \\
\text { splashing on the stuffing box. } \\
\text { Moisture was observed in the } \\
\text { vapor space. }\end{array}$ \\
\hline $\mathrm{F}$ & 34 & $\mathrm{~A}-01$ & $(A)$ & 03/29/02 & WAP & 7 & P02159:05 & Tank condition was normal. \\
\hline $\mathrm{F}$ & 34 & $A-01$ & (A) & 04/03/02 & CCTV & 1 & $808 \mathrm{C}$ & $\begin{array}{l}\text { The conductivity probe was } \\
\text { deployed within the setpoint } \\
\text { limits. }\end{array}$ \\
\hline $\mathrm{F}$ & 34 & A-02 & (A) & 03/29/02 & WAP & 1 & P02159:02 & Tank condition was normal. \\
\hline $\mathrm{F}$ & 34 & A-02 & (A) & 04/03/02 & CCTV & l & $808 \mathrm{C}$ & $\begin{array}{l}\text { The conductivity probe was } \\
\text { deployed within the setpoint } \\
\text { limits. }\end{array}$ \\
\hline $\mathrm{F}$ & 34 & A-03 & $(A)$ & 03/29/02 & WAP & & P02159:13 & Tank condition was normal. \\
\hline $\mathrm{F}$ & 34 & A-03 & (A) & 04/09/02 & CCTV & l & $808 \mathrm{C}$ & $\begin{array}{l}\text { The conductivity probe was } \\
\text { deployed within the setpoint } \\
\text { limits. }\end{array}$ \\
\hline $\mathrm{F}$ & 34 & $\mathrm{~A}-04$ & (A) & 03/29/02 & WAP & & P02159:08 & Tank condition was normal. \\
\hline $\mathrm{F}$ & 34 & A-04 & (A) & 04/03/02 & CCTV & & $808 \mathrm{C}$ & $\begin{array}{l}\text { The magnetically mounted } \\
\text { thermocouple was deployed } \\
\text { within the setpoint limits. }\end{array}$ \\
\hline $\mathrm{F}$ & 34 & P-01 & $(A)$ & 03/29/02 & WAP & l & P02159:10 & Tank condition was normal. \\
\hline
\end{tabular}




\begin{tabular}{|c|c|c|c|c|c|c|c|c|}
\hline \multirow{2}{*}{$\frac{\text { AREA }}{F}$} & \multirow{2}{*}{$\begin{array}{c}\begin{array}{c}\text { TANK OR } \\
\text { ANCILLARY }\end{array} \\
34\end{array}$} & \multicolumn{2}{|c|}{$\frac{\text { ACCESS OPENING }}{(\text { A OR I) }}$} & \multirow{2}{*}{$\frac{\text { DATE }}{03 / 29 / 02}$} & \multicolumn{3}{|c|}{$\begin{array}{l}\text { INSPECTION METHOD } \\
\text { IDENTIFICATION NUMBER }\end{array}$} & \multirow{2}{*}{$\frac{\text { REMARKS }}{\text { Tank condition was normal. }}$} \\
\hline & & P-02 & (A) & & WAP & / & P02159:09 & \\
\hline $\mathrm{F}$ & 34 & P-03 & (A) & 03/28/02 & DP & / & P02137:01-26 & Tank condition was normal. \\
\hline $\mathrm{F}$ & 34 & P-04 & (A) & 03/29/02 & WAP & I & P02159:07 & Tank condition was normal. \\
\hline $\mathrm{F}$ & 34 & P-05 & (A) & 03/29/02 & WAP & / & P02159:06 & Tank condition was normal. \\
\hline $\mathrm{F}$ & 34 & P-06 & (A) & 03/28/02 & DP & / & P02136:01-27 & Tank condition was normal. \\
\hline $\mathrm{F}$ & 34 & P-07 & (A) & 03/29/02 & WAP & / & P02159:04 & Tank condition was normal. \\
\hline $\mathrm{F}$ & 34 & P-08 & (A) & 03/29/02 & WAP & I & P02159:03 & Tank condition was normal. \\
\hline $\mathrm{F}$ & 34 & P-09 & (A) & 03/28/02 & DP & / & P02150:01-27 & Tank condition was normal. \\
\hline $\mathrm{F}$ & 34 & $P-10$ & (A) & 03/28/02 & DP & / & P02138:01-27 & Tank condition was normal. \\
\hline $\mathrm{F}$ & 34 & P-11 & (A) & 03/29/02 & WAP & l & P02159:01 & Tank condition was normal. \\
\hline $\mathrm{F}$ & 34 & P-12 & (A) & 03/29/02 & WAP & / & P02159:15 & Tank condition was normal. \\
\hline $\mathrm{F}$ & 34 & $P-13$ & (A) & 03/28/02 & DP & / & P02135:01-27 & Tank condition was normal. \\
\hline $\mathrm{F}$ & 34 & $P-14$ & (A) & 03/29/02 & WAP & l & P02159:14 & Tank condition was normal. \\
\hline $\mathrm{F}$ & 34 & P-15 & (A) & 03/29/02 & WAP & l & P02159:12 & Tank condition was normal. \\
\hline $\mathrm{F}$ & 34 & P-16 & (A) & 03/29/02 & WAP & I & P02159:11 & Tank condition was normal. \\
\hline $\mathrm{H}$ & 35 & $\mathrm{~A}-01$ & (A) & 04/16/02 & DP & l & P02155:01-27 & Tank condition was normal. \\
\hline $\mathrm{H}$ & 35 & A-02 & (A) & 04/16/02 & CCTV & l & $808 \mathrm{C}$ & $\begin{array}{l}\text { The conductivity probe was } \\
\text { deployed within the setpoint } \\
\text { limits. }\end{array}$ \\
\hline $\mathrm{H}$ & 35 & A-02 & (A) & 04/16/02 & DP & / & P02158:01-27 & Tank condition was normal. \\
\hline $\mathrm{H}$ & 35 & A-03 & (A) & 04/16/02 & CCTV & I & $808 \mathrm{C}$ & $\begin{array}{l}\text { The conductivity probe was } \\
\text { deployed within the setpoint } \\
\text { limits. }\end{array}$ \\
\hline $\mathrm{H}$ & 35 & A-03 & (A) & 04/16/02 & DP & l & P02157:01-27 & Tank condition was normal. \\
\hline $\mathrm{H}$ & 35 & A-04 & (A) & 04/16/02 & CCTV & I & $808 \mathrm{C}$ & $\begin{array}{l}\text { The conductivity probe was } \\
\text { deployed within the setpoint } \\
\text { limits. }\end{array}$ \\
\hline $\mathrm{H}$ & 35 & A-04 & (A) & 04/16/02 & DP & 1 & P02156:01-27 & Tank condition was normal. \\
\hline
\end{tabular}




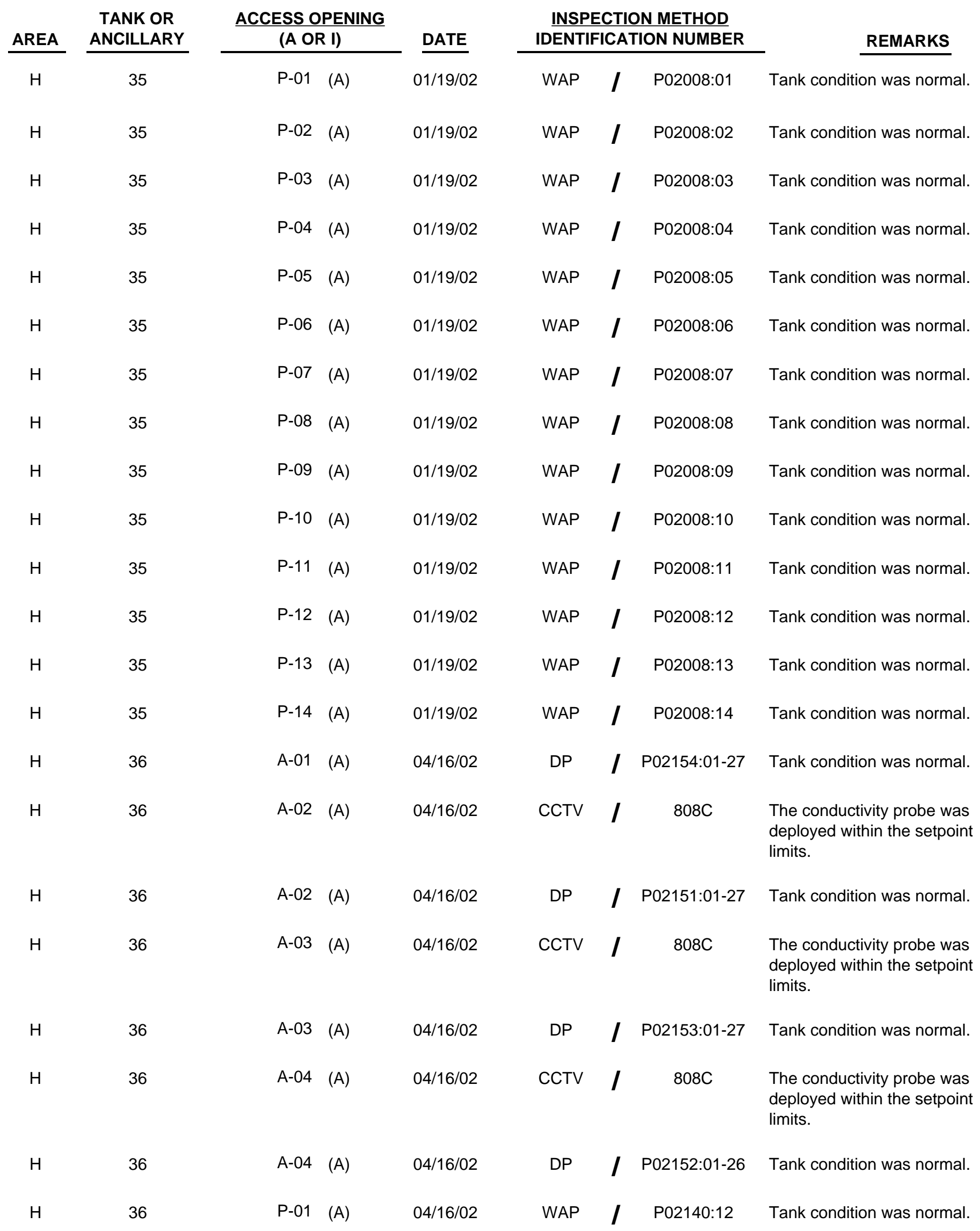




\begin{tabular}{|c|c|c|c|c|c|c|c|c|}
\hline \multirow{2}{*}{$\frac{\text { AREA }}{\mathrm{H}}$} & \multirow{2}{*}{$\begin{array}{c}\begin{array}{c}\text { TANK OR } \\
\text { ANCILLARY }\end{array} \\
36\end{array}$} & \multicolumn{2}{|c|}{$\frac{\text { ACCESS OPENING }}{(A \text { OR I) }}$} & \multirow{2}{*}{$\frac{\text { DATE }}{04 / 16 / 02}$} & \multicolumn{3}{|c|}{$\begin{array}{l}\text { INSPECTION METHOD } \\
\text { IDENTIFICATION NUMBER }\end{array}$} & \multirow{2}{*}{$\frac{\text { REMARKS }}{\text { Tank condition was normal. }}$} \\
\hline & & P-02 & (A) & & WAP & l & P02140:13 & \\
\hline $\mathrm{H}$ & 36 & P-03 & $(\mathrm{A})$ & $04 / 16 / 02$ & WAP & l & P02140:14 & Tank condition was normal. \\
\hline $\mathrm{H}$ & 36 & P-04 & (A) & 04/16/02 & WAP & l & P02140:06 & Tank condition was normal. \\
\hline $\mathrm{H}$ & 36 & P-05 & (A) & 04/16/02 & WAP & l & P02140:07 & Tank condition was normal. \\
\hline $\mathrm{H}$ & 36 & P-06 & (A) & $04 / 16 / 02$ & WAP & l & P02140:08 & Tank condition was normal. \\
\hline $\mathrm{H}$ & 36 & P-07 & $(\mathrm{A})$ & 04/16/02 & WAP & l & P02140:09 & Tank condition was normal. \\
\hline $\mathrm{H}$ & 36 & P-08 & $(\mathrm{A})$ & 04/16/02 & WAP & l & P02140:10 & Tank condition was normal. \\
\hline $\mathrm{H}$ & 36 & P-09 & (A) & 04/16/02 & WAP & l & P02140:11 & Tank condition was normal. \\
\hline $\mathrm{H}$ & 36 & P-10 & (A) & $04 / 16 / 02$ & WAP & l & P02140:01 & Tank condition was normal. \\
\hline $\mathrm{H}$ & 36 & $P-11$ & (A) & $04 / 16 / 02$ & WAP & l & P02140:02 & Tank condition was normal. \\
\hline $\mathrm{H}$ & 36 & P-12 & $(A)$ & $04 / 16 / 02$ & WAP & I & P02140:03 & Tank condition was normal. \\
\hline $\mathrm{H}$ & 36 & $P-13$ & $(A)$ & 04/16/02 & WAP & I & P02140:04 & Tank condition was normal. \\
\hline $\mathrm{H}$ & 36 & $P-14$ & (A) & 04/16/02 & WAP & I & P02140:05 & Tank condition was normal. \\
\hline $\mathrm{H}$ & 37 & & & 03/09/02 & CCTV & l & $809 C$ & $\begin{array}{l}\text { CCTV was used to inspect the } \\
\text { steam line from the transfer jet to } \\
\text { the gang valve house via the } \\
\text { WTS-V-479 valve assembly. } \\
\text { Approximately } 11 \text { feet toward the } \\
\text { gang valve house was inspected, } \\
\text { and no obstructions were } \\
\text { observed. Approximately } 15 \text { feet } \\
\text { toward the jet was inspected, } \\
\text { and no obstructions were } \\
\text { observed. Liquid was observed } \\
\text { at this point. }\end{array}$ \\
\hline $\mathrm{H}$ & 37 & COP 106 & & $07 / 12 / 02$ & CCTV & l & 841 & $\begin{array}{l}\text { The conductivity probe was } \\
\text { deployed within the setpoint } \\
\text { limits. }\end{array}$ \\
\hline $\mathrm{H}$ & 37 & COP 107 & & 07/12/02 & CCTV & l & 841 & $\begin{array}{l}\text { The conductivity probe was } \\
\text { deployed within the setpoint } \\
\text { limits. }\end{array}$ \\
\hline $\mathrm{H}$ & 37 & COP 108 & & $07 / 12 / 02$ & CCTV & l & 841 & $\begin{array}{l}\text { The conductivity probe was } \\
\text { deployed within the setpoint } \\
\text { limits. }\end{array}$ \\
\hline
\end{tabular}




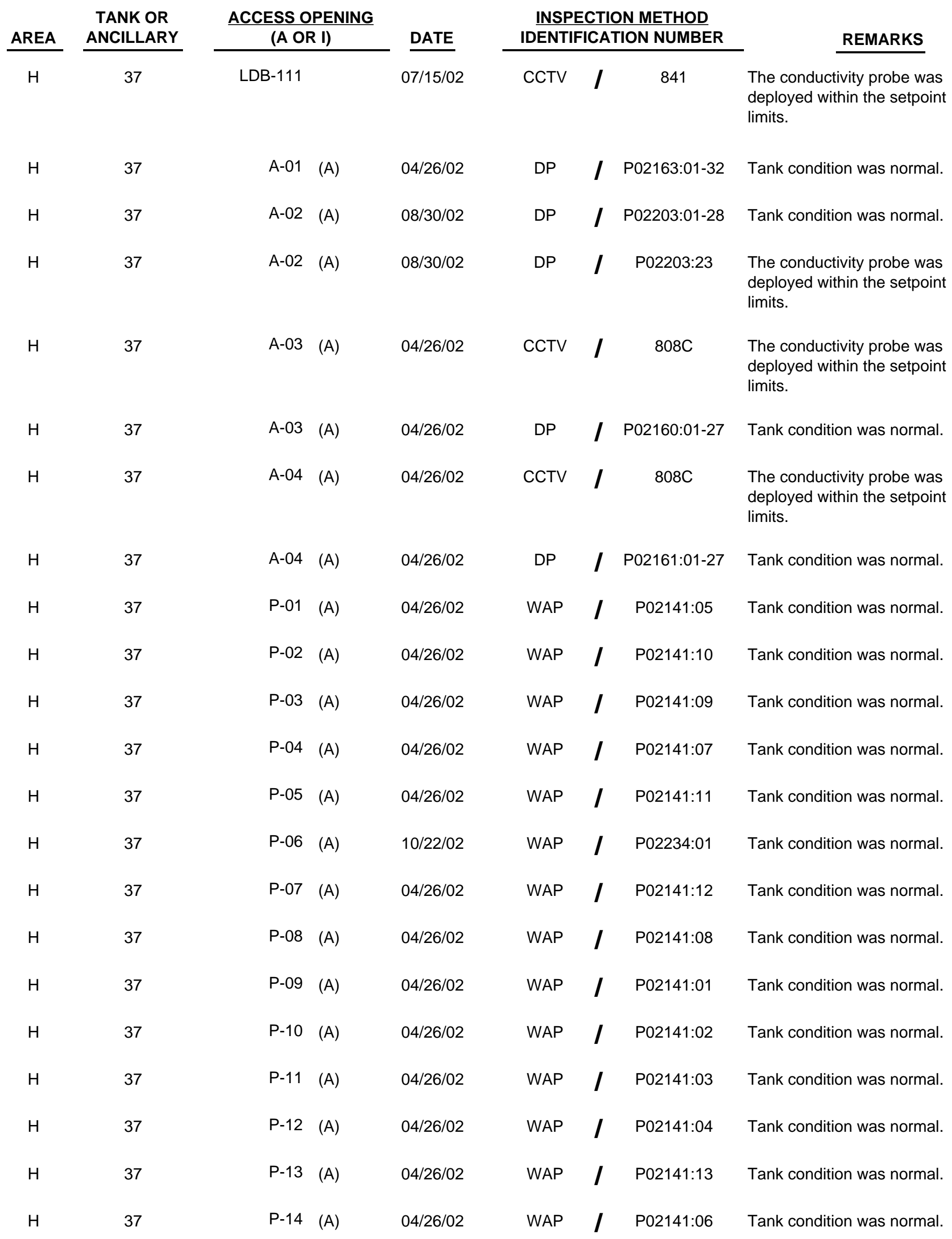




\begin{tabular}{|c|c|c|c|c|c|c|c|c|}
\hline \multirow{2}{*}{$\frac{\text { AREA }}{\mathrm{H}}$} & \multirow{2}{*}{$\begin{array}{c}\text { TANK OR } \\
\text { ANCILLARY } \\
37\end{array}$} & \multicolumn{2}{|c|}{$\frac{\text { ACCESS OPENING }}{(\text { A OR I) }}$} & \multirow{2}{*}{$\frac{\text { DATE }}{03 / 01 / 02}$} & \multicolumn{3}{|c|}{$\frac{\text { INSPECTION METHOD }}{\text { IDENTIFICATION NUMBER }}$} & \multirow{2}{*}{$\begin{array}{l}\text { REMARKS } \\
\text { CCTV was used to leak check } \\
\text { the TTJ. No leaks were } \\
\text { observed. Liquid was observed } \\
\text { coming from the anti-siphon } \\
\text { break. }\end{array}$} \\
\hline & & B-04 & (I) & & CCTV & I & $809 C$ & \\
\hline $\mathrm{H}$ & 37 & B-04 & (I) & 03/19/02 & CCTV & I & $809 \mathrm{C}$ & $\begin{array}{l}\text { Inspection of the waste surface } \\
\text { after it was decanted revealed } \\
\text { uneven surfaces throughout the } \\
\text { tank. The cooling coils were } \\
\text { encrusted with salt } \\
\text { approximately } 2 \text { inches thick } \\
\text { from the waste level to about } 2 \\
\text { feet above the waste. The reel } \\
\text { tape was positioned above the } \\
\text { uneven surface. }\end{array}$ \\
\hline $\mathrm{H}$ & 37 & B-04 & (I) & 03/26/02 & CCTV & I & 809D & $\begin{array}{l}\text { Inspection revealed that no } \\
\text { obstructions were below the reel } \\
\text { tape and that it was positioned } \\
\text { above a pool of liquid. }\end{array}$ \\
\hline $\mathrm{H}$ & 37 & C-01 & (I) & $02 / 14 / 02$ & CCTV & I & $809 B$ & $\begin{array}{l}\text { Inspection was made to } \\
\text { document riser equipment } \\
\text { configuration. A jet and mail box } \\
\text { were observed in the riser. No } \\
\text { unusual conditions were } \\
\text { observed. }\end{array}$ \\
\hline $\mathrm{H}$ & 37 & C-01 & (I) & 02/27/02 & CCTV & I & 823 & $\begin{array}{l}\text { CCTV was used to leak check } \\
\text { the TTJ. No leaks were } \\
\text { observed. }\end{array}$ \\
\hline $\mathrm{H}$ & 37 & C-01 & (I) & 05/07/02 & CCTV & I & 823 & $\begin{array}{l}\text { CCTV was used to verify that no } \\
\text { obstructions existed that would } \\
\text { prevent the dip tube assembly } \\
\text { from being installed in the riser. }\end{array}$ \\
\hline $\mathrm{H}$ & 37 & D-02 & (I) & $03 / 12 / 02$ & CCTV & I & $809 C$ & $\begin{array}{l}\text { Inspection verified that the spare } \\
\text { sample port/thermocouple pipe } \\
\text { was open to the tank vapor } \\
\text { space. }\end{array}$ \\
\hline $\mathrm{H}$ & 37 & D-03 & (I) & 03/12/02 & CCTV & I & $809 C$ & $\begin{array}{l}\text { Inspection verified that the spare } \\
\text { sample port/thermocouple pipe } \\
\text { was open to the tank vapor } \\
\text { space. }\end{array}$ \\
\hline $\mathrm{H}$ & 37 & D-04 & (I) & 03/12/02 & CCTV & I & $809 C$ & $\begin{array}{l}\text { Inspection verified that the spare } \\
\text { sample port/thermocouple pipe } \\
\text { was open to the tank vapor } \\
\text { space. }\end{array}$ \\
\hline $\mathrm{H}$ & 37 & $\mathrm{G}$ & (I) & 03/01/02 & CCTV & I & $809 C$ & $\begin{array}{l}\text { CCTV was used to leak check } \\
\text { the TTJ. No leaks were } \\
\text { observed. }\end{array}$ \\
\hline
\end{tabular}




\section{TANK OR ANCILLARY}

$\mathrm{H}$

37

$\frac{\text { ACCESS OPENING }}{\text { (A OR I) }}$

DATE

G (I)

$12 / 05 / 02$

CCTV /

892

INSPECTION METHOD IDENTIFICATION NUMBER

CCTV / 892

37

G (I)

$12 / 20 / 02$

CCTV

I

892

CCTV

04/01/02

$\mathrm{H} \quad(\mathrm{I})$

$04 / 01 / 02$

$\mathrm{H}$

37

$\mathrm{H} \quad$ (I)

$04 / 11 / 02$

CCTV

I

809E

$\mathrm{H}$

37

H (I)

$11 / 27 / 02$

CCTV

I

887

$\mathrm{H}$

37
H (I)

$12 / 03 / 02$
REMARKS

Inspection of the tank interior after it was decanted revealed that the cooling coils were encrusted with approximately 4 inches of salt. A small area of salt had formed between the coils and was supported by the coils.

Inspection was performed to document condition of the TTJ and tank interior. The TTJ was observed in the raised position with the pickup nozzle above the liquid. No salt was observed on the TTJ. Salt mounds were observed throughout the tank except beneath the TTJ. Salt was attached to the cooling coils above the waste surface.

Inspection documented the tank contents after the tank was decanted.

Inspection of the tank interior after it was decanted revealed that the cooling coils were encrusted with approximately 4 inches of salt. A small area of salt had formed between the coils and was supported by the coils.

Inspection revealed a liquid surface throughout the tank. Salt was attached to the cooling coils above the waste surface.

Inspection revealed an abandoned hose in the southwest side of the tank near the E-02 riser. The waste surface was salt and was irregular throughout the tank. 


\begin{tabular}{|c|c|c|c|c|c|c|c|c|}
\hline \multirow{2}{*}{$\frac{\text { AREA }}{\mathrm{H}}$} & \multirow{2}{*}{$\begin{array}{c}\text { TANK OR } \\
\text { ANCILLARY } \\
37\end{array}$} & \multicolumn{2}{|c|}{$\frac{\text { ACCESS OPENING }}{(A \text { OR I) }}$} & \multirow{2}{*}{$\frac{\text { DATE }}{12 / 05 / 02}$} & \multicolumn{3}{|c|}{$\begin{array}{l}\text { INSPECTION METHOD } \\
\text { IDENTIFICATION NUMBER }\end{array}$} & \multirow[b]{2}{*}{$\begin{array}{l}\text { REMARKS } \\
\text { Inspection of the tank interior } \\
\text { after it was decanted revealed } \\
\text { that the cooling coils were } \\
\text { encrusted with approximately } 4 \\
\text { inches of salt. A small area of } \\
\text { salt had formed between the } \\
\text { coils and was supported by the } \\
\text { coils. }\end{array}$} \\
\hline & & $\mathrm{H}$ & (I) & & CCTV & / & 892 & \\
\hline $\mathrm{H}$ & 38 & GDL & & 09/18/02 & HELIUM & l & HE-02-001 & $\begin{array}{l}\text { Helium tracer testing verified the } \\
\text { integrity of the core pipe of the } \\
\text { gravity drain line from } 242-16 \mathrm{H} \\
\text { Evaporator to Tank } 38 \text {. }\end{array}$ \\
\hline $\mathrm{H}$ & 38 & LDB-01 & & 09/18/02 & CCTV & l & 841 & $\begin{array}{l}\text { The conductivity probe was } \\
\text { deployed within the setpoint } \\
\text { limits. }\end{array}$ \\
\hline $\mathrm{H}$ & 38 & $\mathrm{~A}-01$ & (A) & 01/09/02 & $\mathrm{DP}$ & / & P02073:01-27 & Tank condition was normal. \\
\hline $\mathrm{H}$ & 38 & A-02 & (A) & 01/09/02 & DP & / & P02074:01-27 & Tank condition was normal. \\
\hline $\mathrm{H}$ & 38 & $\mathrm{~A}-02$ & $(\mathrm{~A})$ & 01/09/02 & DP & / & P02074:22 & $\begin{array}{l}\text { The conductivity probe was } \\
\text { deployed within the setpoint } \\
\text { limits. }\end{array}$ \\
\hline $\mathrm{H}$ & 38 & A-03 & (A) & 05/05/02 & DP & / & P02075:22 & $\begin{array}{l}\text { The conductivity probe was } \\
\text { deployed within the setpoint } \\
\text { limits. }\end{array}$ \\
\hline $\mathrm{H}$ & 38 & A-03 & (A) & 05/05/02 & DP & / & P02075:01-26 & Tank condition was normal. \\
\hline $\mathrm{H}$ & 38 & A-04 & $(\mathrm{A})$ & 01/09/02 & DP & / & P02076:01-27 & $\begin{array}{l}\text { Tank condition was normal. } \\
\text { Stains and marks on the annulus } \\
\text { floor and ventilation duct were } \\
\text { due to the inleakage of water. }\end{array}$ \\
\hline $\mathrm{H}$ & 38 & A-04 & (A) & 01/09/02 & DP & / & P02076:22 & $\begin{array}{l}\text { The conductivity probe was } \\
\text { deployed within the setpoint } \\
\text { limits. }\end{array}$ \\
\hline $\mathrm{H}$ & 38 & P-01 & (A) & 01/08/02 & WAP & l & P02003:01 & Tank condition was normal. \\
\hline $\mathrm{H}$ & 38 & P-02 & (A) & 01/08/02 & WAP & l & P02003:02 & Tank condition was normal. \\
\hline $\mathrm{H}$ & 38 & P-03 & (A) & 01/08/02 & WAP & l & P02003:03 & Tank condition was normal. \\
\hline $\mathrm{H}$ & 38 & P-04 & (A) & 01/08/02 & WAP & / & P02003:04 & Tank condition was normal. \\
\hline $\mathrm{H}$ & 38 & $\mathrm{P}-05$ & (A) & 01/08/02 & WAP & / & P02003:05 & Tank condition was normal. \\
\hline $\mathrm{H}$ & 38 & P-06 & (A) & $01 / 08 / 02$ & WAP & l & P02003:06 & Tank condition was normal. \\
\hline
\end{tabular}




\begin{tabular}{|c|c|c|c|c|c|c|c|c|}
\hline \multirow{2}{*}{$\frac{\text { AREA }}{\mathrm{H}}$} & \multirow{2}{*}{$\begin{array}{c}\text { TANK OR } \\
\text { ANCILLARY } \\
38\end{array}$} & \multicolumn{2}{|c|}{$\frac{\text { ACCESS OPENING }}{(\text { A OR I) }}$} & \multirow{2}{*}{$\frac{\text { DATE }}{01 / 08 / 02}$} & \multicolumn{3}{|c|}{$\frac{\text { INSPECTION METHOD }}{\text { IDENTIFICATION NUMBER }}$} & \multirow{2}{*}{$\frac{\text { REMARKS }}{\text { Tank condition was normal. }}$} \\
\hline & & P-07 & (A) & & WAP & / & P02003:07 & \\
\hline $\mathrm{H}$ & 38 & P-08 & (A) & 01/08/02 & WAP & / & P02003:08 & Tank condition was normal. \\
\hline $\mathrm{H}$ & 38 & P-08 & (A) & 02/06/02 & CCTV & / & 809B & $\begin{array}{l}\text { Inspection confirmed that } \\
\text { rainwater was entering the } \\
\text { annulus via penetration line } \\
\text { designated SP through a failed } \\
\text { seal. }\end{array}$ \\
\hline $\mathrm{H}$ & 38 & P-09 & (A) & 01/08/02 & WAP & / & P02003:09 & Tank condition was normal. \\
\hline $\mathrm{H}$ & 38 & P-10 & (A) & 01/08/02 & WAP & l & P02003:10 & $\begin{array}{l}\text { Tank condition was normal. } \\
\text { Stains and marks on the annulus } \\
\text { floor had increased since last } \\
\text { inspected due to the inleakage of } \\
\text { water. }\end{array}$ \\
\hline $\mathrm{H}$ & 38 & $\mathrm{P}-11$ & (A) & 01/08/02 & WAP & / & P02003:11 & $\begin{array}{l}\text { Tank condition was normal. } \\
\text { Stains and marks on the annulus } \\
\text { floor and ventilation duct had } \\
\text { increased since last inspected } \\
\text { due to the inleakage of water. }\end{array}$ \\
\hline $\mathrm{H}$ & 38 & $P-11$ & (A) & 02/06/02 & CCTV & / & 809B & $\begin{array}{l}\text { Inspection of the area beneath } \\
\text { penetration lines designated WF, } \\
\text { WS1, SJJ, and SJ1 revealed no } \\
\text { unusual conditions. The annulus } \\
\text { floor was dry. }\end{array}$ \\
\hline $\mathrm{H}$ & 38 & P-12 & (A) & 01/08/02 & WAP & / & P02003:12 & $\begin{array}{l}\text { Tank condition was normal. } \\
\text { Stains and marks on the annulus } \\
\text { floor and ventilation duct had } \\
\text { increased since last inspected } \\
\text { due to the inleakage of water. }\end{array}$ \\
\hline $\mathrm{H}$ & 38 & P-13 & (A) & 01/08/02 & WAP & / & P02003:13 & $\begin{array}{l}\text { Tank condition was normal. } \\
\text { Stains and marks on the annulus } \\
\text { floor and ventilation duct had } \\
\text { increased since last inspected } \\
\text { due to the inleakage of water. }\end{array}$ \\
\hline $\mathrm{H}$ & 38 & P-14 & (A) & 01/08/02 & WAP & / & P02003:14 & Tank condition was normal. \\
\hline $\mathrm{H}$ & 39 & & & 04/21/02 & CCTV & / & $809 E$ & $\begin{array}{l}\text { CCTV was used to determine if } \\
\text { the gang valve house floor drain } \\
\text { was plugged. The line was } \\
\text { plugged approximately } 3 \text { feet } \\
\text { from the opening. }\end{array}$ \\
\hline
\end{tabular}




\begin{tabular}{|c|c|c|c|c|c|c|c|c|}
\hline \multirow{2}{*}{$\frac{\text { AREA }}{H}$} & \multirow{2}{*}{$\begin{array}{c}\begin{array}{c}\text { TANK OR } \\
\text { ANCILLARY }\end{array} \\
39\end{array}$} & \multicolumn{2}{|c|}{$\frac{\text { ACCESS OPENING }}{\text { (A OR I) }}$} & \multirow{2}{*}{$\frac{\text { DATE }}{04 / 29 / 02}$} & \multicolumn{3}{|c|}{$\begin{array}{l}\text { INSPECTION METHOD } \\
\text { IDENTIFICATION NUMBER }\end{array}$} & \multirow{2}{*}{$\begin{array}{l}\text { REMARKS } \\
\text { Inspection revealed that } \\
\text { corrosion particles and dried } \\
\text { mud prevented the conductiv } \\
\text { probe from being properly } \\
\text { deployed. }\end{array}$} \\
\hline & & LDB-01 & & & CCTV & / & $807 \mathrm{~A}$ & \\
\hline $\mathrm{H}$ & 39 & LDB-01 & & 05/01/02 & CCTV & l & $807 \mathrm{~A}$ & $\begin{array}{l}\text { The conductivity probe was } \\
\text { deployed within the setpoint } \\
\text { limits. }\end{array}$ \\
\hline $\mathrm{H}$ & 39 & LDB-01 & & $11 / 05 / 02$ & CCTV & l & 841 & $\begin{array}{l}\text { The conductivity probe was } \\
\text { deployed within the setpoint } \\
\text { limits. }\end{array}$ \\
\hline $\mathrm{H}$ & 39 & $A-01$ & (A) & 01/09/02 & DP & I & P02077:01-29 & Tank condition was normal. \\
\hline $\mathrm{H}$ & 39 & A-02 & (A) & 01/09/02 & DP & I & P02078:01-26 & Tank condition was normal. \\
\hline $\mathrm{H}$ & 39 & A-02 & (A) & 01/09/02 & DP & / & P02078:22 & $\begin{array}{l}\text { The conductivity probe was } \\
\text { deployed within the setpoint } \\
\text { limits. }\end{array}$ \\
\hline $\mathrm{H}$ & 39 & A-03 & (A) & 01/09/02 & DP & I & P02079:01-27 & Tank condition was normal. \\
\hline $\mathrm{H}$ & 39 & $\mathrm{~A}-03$ & (A) & 01/09/02 & DP & / & P02079:22 & $\begin{array}{l}\text { The conductivity probe was } \\
\text { deployed within the setpoint } \\
\text { limits. }\end{array}$ \\
\hline $\mathrm{H}$ & 39 & A-04 & (A) & 01/09/02 & DP & / & P02080:01-28 & Tank condition was normal. \\
\hline $\mathrm{H}$ & 39 & A-04 & (A) & 01/09/02 & DP & l & P02080:22 & $\begin{array}{l}\text { The conductivity probe was } \\
\text { deployed within the setpoint } \\
\text { limits. }\end{array}$ \\
\hline $\mathrm{H}$ & 39 & P-01 & (A) & 01/08/02 & WAP & / & P02002:01 & Tank condition was normal. \\
\hline $\mathrm{H}$ & 39 & P-02 & (A) & 01/08/02 & WAP & / & P02002:02 & Tank condition was normal. \\
\hline $\mathrm{H}$ & 39 & P-03 & (A) & 01/08/02 & WAP & / & P02002:03 & Tank condition was normal. \\
\hline $\mathrm{H}$ & 39 & P-04 & (A) & 01/08/02 & WAP & I & P02002:04 & Tank condition was normal. \\
\hline $\mathrm{H}$ & 39 & P-05 & (A) & 01/08/02 & WAP & / & P02002:05 & Tank condition was normal. \\
\hline $\mathrm{H}$ & 39 & P-06 & (A) & 01/08/02 & WAP & / & P02002:06 & Tank condition was normal. \\
\hline $\mathrm{H}$ & 39 & P-07 & (A) & 01/08/02 & WAP & / & P02002:07 & Tank condition was normal. \\
\hline $\mathrm{H}$ & 39 & P-08 & (A) & 01/08/02 & WAP & I & P02002:08 & Tank condition was normal. \\
\hline $\mathrm{H}$ & 39 & P-09 & (A) & $01 / 08 / 02$ & WAP & l & P02002:09 & Tank condition was normal. \\
\hline
\end{tabular}




\begin{tabular}{|c|c|c|c|c|c|c|c|c|}
\hline \multirow{2}{*}{$\frac{\text { AREA }}{\mathrm{H}}$} & \multirow{2}{*}{$\begin{array}{c}\begin{array}{c}\text { TANK OR } \\
\text { ANCILLARY }\end{array} \\
39\end{array}$} & \multicolumn{2}{|c|}{$\frac{\text { ACCESS OPENING }}{(A \text { OR I) }}$} & \multirow{2}{*}{$\frac{\text { DATE }}{01 / 08 / 02}$} & \multicolumn{3}{|c|}{$\begin{array}{l}\text { INSPECTION METHOD } \\
\text { IDENTIFICATION NUMBER }\end{array}$} & \multirow{2}{*}{$\frac{\text { REMARKS }}{\text { Tank condition was normal. }}$} \\
\hline & & P-10 & (A) & & WAP & I & P02002:10 & \\
\hline $\mathrm{H}$ & 39 & $P-11$ & (A) & 01/08/02 & WAP & I & P02002:11 & Tank condition was normal. \\
\hline $\mathrm{H}$ & 39 & $\mathrm{P}-12$ & (A) & 01/08/02 & WAP & / & P02002:12 & Tank condition was normal. \\
\hline $\mathrm{H}$ & 39 & $P-13$ & (A) & 01/08/02 & WAP & / & P02002:13 & Tank condition was normal. \\
\hline $\mathrm{H}$ & 39 & P-14 & (A) & 01/08/02 & WAP & / & P02002:14 & Tank condition was normal. \\
\hline $\mathrm{H}$ & 39 & C-01 & (I) & 04/10/02 & CCTV & / & $822 \mathrm{~A}$ & $\begin{array}{l}\text { CCTV was used to leak check } \\
\text { the transfer jet. No leaks were } \\
\text { observed. }\end{array}$ \\
\hline $\mathrm{H}$ & 39 & $\mathrm{H}$ & (I) & $04 / 12 / 02$ & CCTV & / & $822 \mathrm{~A}$ & $\begin{array}{l}\text { Inspection verified liquid flowing } \\
\text { from the anti-siphon break during } \\
\text { flushing. The reel tape was } \\
\text { operating properly. }\end{array}$ \\
\hline $\mathrm{H}$ & 40 & LDB-01 & & 03/09/02 & CCTV & / & $807 \mathrm{~A}$ & $\begin{array}{l}\text { The conductivity probe was } \\
\text { deployed within the setpoint } \\
\text { limits. }\end{array}$ \\
\hline $\mathrm{H}$ & 40 & LDB-02 & & 03/09/02 & CCTV & l & $807 \mathrm{~A}$ & $\begin{array}{l}\text { The conductivity probe was } \\
\text { deployed within the setpoint } \\
\text { limits. }\end{array}$ \\
\hline $\mathrm{H}$ & 40 & $A-01$ & (A) & 08/14/02 & WAP & I & P02200:12 & Tank condition was normal. \\
\hline $\mathrm{H}$ & 40 & A-02 & (A) & 01/08/02 & CCTV & l & 808 & $\begin{array}{l}\text { The conductivity probe was } \\
\text { deployed within the setpoint } \\
\text { limits. }\end{array}$ \\
\hline $\mathrm{H}$ & 40 & A-02 & (A) & 08/14/02 & WAP & / & P02200:02 & Tank condition was normal. \\
\hline $\mathrm{H}$ & 40 & A-03 & (A) & 01/08/02 & CCTV & / & 808 & $\begin{array}{l}\text { The conductivity probe was } \\
\text { deployed within the setpoint } \\
\text { limits. }\end{array}$ \\
\hline $\mathrm{H}$ & 40 & A-03 & (A) & 08/14/02 & WAP & / & P02200:06 & Tank condition was normal. \\
\hline $\mathrm{H}$ & 40 & A-04 & (A) & 01/08/02 & CCTV & l & 808 & $\begin{array}{l}\text { The conductivity probe was } \\
\text { deployed within the setpoint } \\
\text { limits. }\end{array}$ \\
\hline $\mathrm{H}$ & 40 & A-04 & (A) & $08 / 14 / 02$ & WAP & / & P02200:09 & Tank condition was normal. \\
\hline $\mathrm{H}$ & 40 & Center & (A) & 05/20/02 & CCTV & / & 852 & $\begin{array}{l}\text { Inspection of the center annulus } \\
\text { standpipe revealed loose } \\
\text { sand-like pluggage } \\
\text { approximately } 31^{\prime} 6 \text { " from the top } \\
\text { of the standpipe. }\end{array}$ \\
\hline
\end{tabular}




\begin{tabular}{|c|c|c|c|c|c|c|c|c|}
\hline \multirow{2}{*}{$\frac{\text { AREA }}{\mathrm{H}}$} & \multirow{2}{*}{$\begin{array}{c}\text { TANK OR } \\
\text { ANCILLARY } \\
40\end{array}$} & \multicolumn{2}{|c|}{$\frac{\text { ACCESS OPENING }}{(\text { A OR I) }}$} & \multirow{2}{*}{$\frac{\text { DATE }}{08 / 14 / 02}$} & \multicolumn{3}{|c|}{$\begin{array}{l}\text { INSPECTION METHOD } \\
\text { IDENTIFICATION NUMBER }\end{array}$} & \multirow{2}{*}{$\frac{\text { REMARKS }}{\text { Tank condition was normal. }}$} \\
\hline & & P-01 & (A) & & WAP & / & P02200:01 & \\
\hline $\mathrm{H}$ & 40 & P-02 & (A) & 08/16/02 & DP & / & P02199:01-27 & Tank condition was normal. \\
\hline $\mathrm{H}$ & 40 & P-03 & (A) & 08/14/02 & WAP & / & P02200:13 & Tank condition was normal. \\
\hline $\mathrm{H}$ & 40 & P-04 & (A) & 08/14/02 & WAP & / & P02200:03 & Tank condition was normal. \\
\hline $\mathrm{H}$ & 40 & P-05 & (A) & 08/16/02 & DP & l & P02197:01-27 & Tank condition was normal. \\
\hline $\mathrm{H}$ & 40 & P-06 & (A) & $08 / 14 / 02$ & WAP & / & P02200:04 & Tank condition was normal. \\
\hline $\mathrm{H}$ & 40 & P-07 & (A) & 08/14/02 & WAP & / & P02200:05 & Tank condition was normal. \\
\hline $\mathrm{H}$ & 40 & P-08 & (A) & 08/16/02 & DP & / & P02195:01-27 & Tank condition was normal. \\
\hline $\mathrm{H}$ & 40 & P-09 & (A) & 08/14/02 & WAP & / & P02200:07 & Tank condition was normal. \\
\hline $\mathrm{H}$ & 40 & $P-10$ & (A) & 08/14/02 & WAP & l & P02200:08 & Tank condition was normal. \\
\hline $\mathrm{H}$ & 40 & $P-11$ & (A) & 08/16/02 & DP & l & P02196:01-27 & Tank condition was normal. \\
\hline $\mathrm{H}$ & 40 & $\mathrm{P}-12$ & (A) & 08/14/02 & WAP & / & P02200:10 & Tank condition was normal. \\
\hline $\mathrm{H}$ & 40 & $P-13$ & (A) & 08/14/02 & WAP & / & P02200:11 & Tank condition was normal. \\
\hline $\mathrm{H}$ & 40 & P-14 & (A) & 08/16/02 & DP & / & P02198:01-27 & Tank condition was normal. \\
\hline $\mathrm{H}$ & 40 & B-01 & (I) & 02/16/02 & CCTV & I & 813 & $\begin{array}{l}\text { Inspection was performed to } \\
\text { document movement of coils and } \\
\text { thermowells during operation of } \\
\text { slurry pumps B2, B6, and G at } \\
\text { maximum RPM's. Some } \\
\text { movement of coils near the B6 } \\
\text { pump was observed. }\end{array}$ \\
\hline $\mathrm{H}$ & 40 & B-03 & (I) & 02/16/02 & CCTV & I & 813 & $\begin{array}{l}\text { Inspection was performed to } \\
\text { document movement of coils and } \\
\text { thermowells during operation of } \\
\text { slurry pumps B2, B6, and G at } \\
\text { maximum RPM's. Significant } \\
\text { movement of coils near } \\
\text { operating pumps was observed. } \\
\text { The D2 thermowell moved } \\
\text { laterally approximately } 2 \text { feet at } \\
\text { the waste level. }\end{array}$ \\
\hline $\mathrm{H}$ & 41 & LDB-01 & & 04/10/02 & CCTV & I & $807 A$ & $\begin{array}{l}\text { The conductivity probe was } \\
\text { deployed within the setpoint } \\
\text { limits. }\end{array}$ \\
\hline
\end{tabular}




\begin{tabular}{|c|c|c|c|c|c|c|c|c|}
\hline \multirow{2}{*}{$\frac{\text { AREA }}{\mathrm{H}}$} & \multirow{2}{*}{$\begin{array}{c}\text { TANK OR } \\
\text { ANCILLARY } \\
41\end{array}$} & \multicolumn{2}{|c|}{$\frac{\text { ACCESS OPENING }}{(A \text { OR I) }}$} & \multirow{2}{*}{$\frac{\text { DATE }}{03 / 06 / 02}$} & \multicolumn{3}{|c|}{$\frac{\text { INSPECTION METHOD }}{\text { IDENTIFICATION NUMBER }}$} & \multirow{2}{*}{$\begin{array}{l}\text { REMARKS } \\
\text { The conductivity probe was } \\
\text { deployed within the setpoint } \\
\text { limits. }\end{array}$} \\
\hline & & LDB-02 & & & CCTV & l & $807 \mathrm{~A}$ & \\
\hline $\mathrm{H}$ & 41 & A-02 & (A) & 01/08/02 & CCTV & / & 808 & $\begin{array}{l}\text { The conductivity probe was } \\
\text { deployed within the setpoint } \\
\text { limits. }\end{array}$ \\
\hline $\mathrm{H}$ & 41 & A-02 & $(\mathrm{A})$ & 09/12/02 & DP & / & P02226:01-29 & Tank condition was normal. \\
\hline $\mathrm{H}$ & 41 & A-03 & (A) & 01/08/02 & CCTV & / & 808 & $\begin{array}{l}\text { The conductivity probe was } \\
\text { deployed within the setpoint } \\
\text { limits. }\end{array}$ \\
\hline $\mathrm{H}$ & 41 & $\mathrm{~A}-03$ & $(\mathrm{~A})$ & 04/05/02 & CCTV & / & 809D & $\begin{array}{l}\text { Inspection was made after a } \\
\text { conductivity probe alarm was } \\
\text { received. Water observed on } \\
\text { the annulus floor had entered the } \\
\text { annulus via a failed seal at the } \\
\text { penetration line designated SP. }\end{array}$ \\
\hline $\mathrm{H}$ & 41 & A-03 & (A) & 04/09/02 & CCTV & / & 809E & $\begin{array}{l}\text { Inspection revealed water from a } \\
\text { broken domestic water line was } \\
\text { entering the annulus via a failed } \\
\text { packing seal at penetration line } \\
\text { designated SP. }\end{array}$ \\
\hline $\mathrm{H}$ & 41 & A-03 & (A) & 09/12/02 & DP & / & P02228:01-28 & Tank condition was normal. \\
\hline $\mathrm{H}$ & 41 & $A-04$ & $(\mathrm{~A})$ & 01/08/02 & CCTV & / & 808 & $\begin{array}{l}\text { The conductivity probe was } \\
\text { deployed within the setpoint } \\
\text { limits. }\end{array}$ \\
\hline $\mathrm{H}$ & 41 & $A-04$ & (A) & 09/12/02 & DP & / & P02227:01-27 & Tank condition was normal. \\
\hline $\mathrm{H}$ & 41 & P-01 & (A) & $10 / 24 / 02$ & WAP & l & P02230:02 & Tank condition was normal. \\
\hline $\mathrm{H}$ & 41 & P-02 & (A) & $10 / 24 / 02$ & WAP & / & P02230:03 & Tank condition was normal. \\
\hline $\mathrm{H}$ & 41 & P-03 & (A) & $10 / 24 / 02$ & WAP & / & P02230:04 & Tank condition was normal. \\
\hline $\mathrm{H}$ & 41 & $\mathrm{P}-04$ & (A) & $10 / 24 / 02$ & WAP & l & P02230:05 & Tank condition was normal. \\
\hline $\mathrm{H}$ & 41 & P-05 & (A) & $10 / 24 / 02$ & WAP & / & P02230:06 & Tank condition was normal. \\
\hline $\mathrm{H}$ & 41 & P-06 & (A) & $10 / 24 / 02$ & WAP & I & P02230:07 & Tank condition was normal. \\
\hline $\mathrm{H}$ & 41 & P-07 & (A) & $10 / 24 / 02$ & WAP & I & P02230:08 & Tank condition was normal. \\
\hline $\mathrm{H}$ & 41 & P-08 & (A) & 06/06/02 & CCTV & I & 830 & $\begin{array}{l}\text { Inspection was made to identify } \\
\text { location of water inleakage. No } \\
\text { inleakage was observed. }\end{array}$ \\
\hline
\end{tabular}




\begin{tabular}{|c|c|c|c|c|c|c|}
\hline \multirow{2}{*}{$\frac{\text { AREA }}{\mathrm{H}}$} & \multirow{2}{*}{$\begin{array}{c}\text { TANK OR } \\
\text { ANCILLARY } \\
41\end{array}$} & $\frac{\text { ACCESS OPENING }}{(\text { A OR I) }}$ & \multirow{2}{*}{$\frac{\text { DATE }}{10 / 24 / 02}$} & \multicolumn{2}{|c|}{$\begin{array}{l}\text { INSPECTION METHOD } \\
\text { IDENTIFICATION NUMBER }\end{array}$} & \multirow{2}{*}{$\frac{\text { REMARKS }}{\text { Tank condition was normal. }}$} \\
\hline & & P-08 (A) & & WAP & P02230:09 & \\
\hline $\mathrm{H}$ & 41 & P-09 (A) & $10 / 24 / 02$ & WAP & P02230:10 & Tank condition was normal. \\
\hline $\mathrm{H}$ & 41 & $P-10 \quad(A)$ & $10 / 24 / 02$ & WAP & P02230:11 & Tank condition was normal. \\
\hline $\mathrm{H}$ & 41 & $\mathrm{P}-11 \quad(\mathrm{~A})$ & $10 / 24 / 02$ & WAP & P02230:12 & Tank condition was normal. \\
\hline $\mathrm{H}$ & 41 & $\mathrm{P}-12 \quad(\mathrm{~A})$ & $10 / 24 / 02$ & WAP & P02230:13 & Tank condition was normal. \\
\hline $\mathrm{H}$ & 41 & $\mathrm{P}-13 \quad(\mathrm{~A})$ & $10 / 24 / 02$ & WAP & P02230:14 & Tank condition was normal. \\
\hline $\mathrm{H}$ & 41 & $\mathrm{P}-14 \quad(\mathrm{~A})$ & $10 / 24 / 02$ & WAP & P02230:01 & Tank condition was normal. \\
\hline $\mathrm{H}$ & 41 & B-02 (I) & 05/04/02 & CCTV & 824 & $\begin{array}{l}\text { Inspection revealed abandoned } \\
\text { steel tapes on the waste surface } \\
\text { beneath the riser. }\end{array}$ \\
\hline
\end{tabular}

CCTV was used to document tank conditions and verify that no obstructions existed beneath the $\mathrm{B} 2, \mathrm{~B} 4$, and $\mathrm{E} 1$ risers. No reel tapes or obstructions were visible beneath the $\mathrm{B} 2$ and $\mathrm{B} 4$ risers. The area beneath the $\mathrm{E} 1$ riser was not visible. Abandoned steel tapes were observed beneath the $\mathrm{G}$ riser.

$\mathrm{H}$

$\mathrm{H}$

41

$\mathrm{H}$

41

41
$\mathrm{H}$
41
B-04

(I)

05/04/02

C-01

(I)

07/13/02

CCTV /

824

CCTV /

871

824

CCTV /
G (I)
08/22/02 


\begin{tabular}{|c|c|c|c|c|c|c|c|c|}
\hline \multirow{2}{*}{$\frac{\text { AREA }}{\mathrm{H}}$} & \multirow{2}{*}{$\begin{array}{c}\text { TANK OR } \\
\text { ANCILLARY } \\
41\end{array}$} & \multicolumn{2}{|c|}{$\frac{\text { ACCESS OPENING }}{(\text { A OR I) }}$} & \multirow{2}{*}{$\frac{\text { DATE }}{08 / 24 / 02}$} & \multicolumn{3}{|c|}{$\begin{array}{l}\text { INSPECTION METHOD } \\
\text { IDENTIFICATION NUMBER }\end{array}$} & \multirow{2}{*}{$\begin{array}{l}\text { REMARKS } \\
\text { CCTV revealed leakage from the } \\
\text { nozzle box on the C1 transfer } \\
\text { pump. }\end{array}$} \\
\hline & & G & (I) & & CCTV & I & 843 & \\
\hline $\mathrm{H}$ & 41 & G & (I) & 08/31/02 & CCTV & / & 843 & $\begin{array}{l}\text { Inspection was made to } \\
\text { document any salt accumulation } \\
\text { on the } \mathrm{C} 1 \text { transfer pump. No } \\
\text { salt formations were observed. }\end{array}$ \\
\hline $\mathrm{H}$ & 41 & G & (I) & 09/14/02 & CCTV & I & 869 & $\begin{array}{l}\text { Inspection documented the } \\
\text { conditions of the tank. Exposed } \\
\text { salt cake was observed. }\end{array}$ \\
\hline $\mathrm{H}$ & 41 & $\mathrm{G}$ & $(\mathrm{I})$ & 09/19/02 & CCTV & / & 830 & $\begin{array}{l}\text { CCTV documented the } \\
\text { conditions of the transfer pump } \\
\text { in the } \mathrm{C} 1 \text { riser and waste } \\
\text { surface. The salt well formed } \\
\text { around the pump was caving in. }\end{array}$ \\
\hline $\mathrm{H}$ & 41 & $\mathrm{G}$ & $(\mathrm{I})$ & 09/22/02 & CCTV & / & 869 & $\begin{array}{l}\text { Inspection revealed that the salt } \\
\text { mound was visible throughout } \\
\text { the entire tank. No liquid was } \\
\text { visible. The C1 transfer pump } \\
\text { was free of any salt } \\
\text { accumulation. }\end{array}$ \\
\hline $\mathrm{H}$ & 41 & G & $(\mathrm{I})$ & $10 / 21 / 02$ & CCTV & / & 874 & $\begin{array}{l}\text { CCTV was used to document the } \\
\text { conditions of the tank. Salt was } \\
\text { observed throughout the tank. }\end{array}$ \\
\hline $\mathrm{H}$ & 42 & LDB-01 & & 05/23/02 & CCTV & I & $807 A$ & $\begin{array}{l}\text { The conductivity probe was } \\
\text { deployed within the setpoint } \\
\text { limits. }\end{array}$ \\
\hline $\mathrm{H}$ & 42 & VB & & $10 / 19 / 02$ & CCTV & l & 854 & $\begin{array}{l}\text { Inspection documented the } \\
\text { conditions of the valve box. No } \\
\text { evidence of leakage or unusual } \\
\text { conditions were observed. A } \\
\text { small amount of liquid was } \\
\text { observed on the floor of the VB. } \\
\text { The conductivity probes were } \\
\text { observed extending below the } \\
\text { standpipes possibly contacting } \\
\text { the floor. }\end{array}$ \\
\hline $\mathrm{H}$ & 42 & A-01 & (A) & 08/18/02 & WAP & / & P02201:11 & Tank condition was normal. \\
\hline $\mathrm{H}$ & 42 & A-02 & (A) & 01/08/02 & CCTV & / & 808 & $\begin{array}{l}\text { The conductivity probe was } \\
\text { deployed within the setpoint } \\
\text { limits. }\end{array}$ \\
\hline $\mathrm{H}$ & 42 & A-02 & (A) & $01 / 12 / 02$ & WAP & I & P02086:01 & Tank condition was normal. \\
\hline $\mathrm{H}$ & 42 & A-02 & (A) & 08/18/02 & WAP & l & P02201:08 & Tank condition was normal. \\
\hline
\end{tabular}




\begin{tabular}{|c|c|c|c|c|c|c|c|c|}
\hline \multirow{2}{*}{$\frac{\text { AREA }}{\mathrm{H}}$} & \multirow{2}{*}{$\begin{array}{c}\begin{array}{c}\text { TANK OR } \\
\text { ANCILLARY }\end{array} \\
42\end{array}$} & \multicolumn{2}{|c|}{$\frac{\text { ACCESS OPENING }}{(\text { A OR I) }}$} & \multirow{2}{*}{$\frac{\text { DATE }}{01 / 08 / 02}$} & \multicolumn{3}{|c|}{$\begin{array}{l}\text { INSPECTION METHOD } \\
\text { IDENTIFICATION NUMBER }\end{array}$} & \multirow{2}{*}{$\begin{array}{l}\text { REMARKS } \\
\text { The conductivity probe was } \\
\text { deployed within the setpoint } \\
\text { limits. }\end{array}$} \\
\hline & & A-03 & (A) & & CCTV & l & 808 & \\
\hline $\mathrm{H}$ & 42 & A-03 & (A) & 08/18/02 & WAP & l & P02201:05 & $\begin{array}{l}\text { Tank condition was normal. } \\
\text { Stains observed on the } \\
\text { secondary vessel wall were due } \\
\text { to inleakage of water. }\end{array}$ \\
\hline $\mathrm{H}$ & 42 & A-04 & (A) & 01/08/02 & CCTV & I & 808 & $\begin{array}{l}\text { The conductivity probe was } \\
\text { deployed within the setpoint } \\
\text { limits. }\end{array}$ \\
\hline $\mathrm{H}$ & 42 & A-04 & (A) & $12 / 12 / 02$ & WAP & I & P02244:01 & Tank condition was normal. \\
\hline $\mathrm{H}$ & 42 & P-01 & (A) & 08/18/02 & WAP & I & P02201:10 & Tank condition was normal. \\
\hline $\mathrm{H}$ & 42 & P-02 & (A) & 08/18/02 & WAP & / & P02201:09 & Tank condition was normal. \\
\hline $\mathrm{H}$ & 42 & P-03 & (A) & 08/18/02 & DP & l & P02183:01-27 & Tank condition was normal. \\
\hline $\mathrm{H}$ & 42 & P-04 & (A) & $11 / 21 / 02$ & WAP & l & P02238:03 & $\begin{array}{l}\text { Tank condition was normal. } \\
\text { Stains and marks on the } \\
\text { secondary vessel wall and } \\
\text { ventilation duct were due to } \\
\text { inleakage of water. }\end{array}$ \\
\hline $\mathrm{H}$ & 42 & P-05 & (A) & 08/18/02 & WAP & 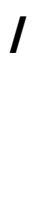 & P02201:02 & $\begin{array}{l}\text { Tank condition was normal. } \\
\text { Stains and marks on the } \\
\text { secondary vessel wall and } \\
\text { ventilation duct were due to } \\
\text { inleakage of water. }\end{array}$ \\
\hline $\mathrm{H}$ & 42 & P-06 & (A) & 08/18/02 & WAP & l & P02201:06 & $\begin{array}{l}\text { Tank condition was normal. } \\
\text { Stains and marks on the } \\
\text { secondary vessel wall and } \\
\text { ventilation duct were due to } \\
\text { inleakage of water. }\end{array}$ \\
\hline $\mathrm{H}$ & 42 & P-07 & $(\mathrm{A})$ & 08/18/02 & DP & l & P02184:01-28 & $\begin{array}{l}\text { Tank condition was normal. } \\
\text { Stains and marks on the } \\
\text { secondary vessel wall and } \\
\text { ventilation duct were due to } \\
\text { inleakage of water. }\end{array}$ \\
\hline $\mathrm{H}$ & 42 & P-08 & (A) & 08/18/02 & WAP & l & P02201:03 & $\begin{array}{l}\text { Tank condition was normal. } \\
\text { Stains and marks on the } \\
\text { secondary vessel wall and } \\
\text { ventilation duct were due to } \\
\text { inleakage of water. }\end{array}$ \\
\hline $\mathrm{H}$ & 42 & P-09 & (A) & $11 / 21 / 02$ & WAP & 1 & P02238:04 & Tank condition was normal. \\
\hline
\end{tabular}




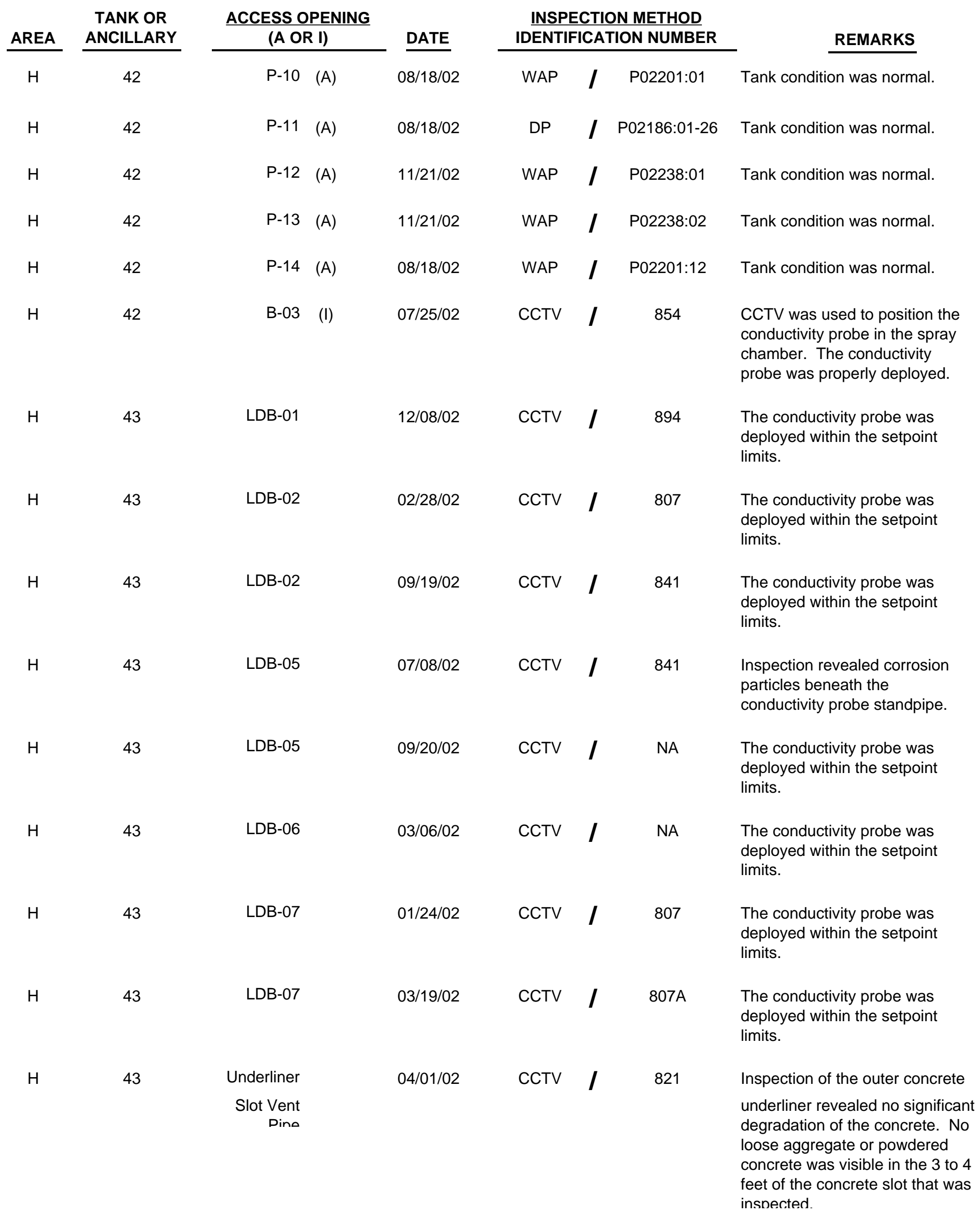




\begin{tabular}{|c|c|c|c|c|c|c|c|c|}
\hline \multirow{2}{*}{ AREA } & \multirow{2}{*}{$\begin{array}{c}\begin{array}{c}\text { TANK OR } \\
\text { ANCILLARY }\end{array} \\
43\end{array}$} & \multicolumn{2}{|c|}{$\frac{\text { ACCESS OPENING }}{(A \text { OR I) }}$} & \multirow{2}{*}{$\frac{\text { DATE }}{04 / 27 / 02}$} & \multicolumn{3}{|c|}{$\begin{array}{l}\text { INSPECTION METHOD } \\
\text { IDENTIFICATION NUMBER }\end{array}$} & \multirow{2}{*}{$\begin{array}{l}\text { REMARKS } \\
\text { Inspection of the outer concrete } \\
\text { underliner revealed no significant } \\
\text { degradation of the concrete, and } \\
\text { only general corrosion of the } \\
\text { carbon steel secondary liner. }\end{array}$} \\
\hline & & $\begin{array}{r}\text { Underliner } \\
\text { Slot Vent } \\
\text { Pipe }\end{array}$ & & & CCTV & / & $809 \mathrm{~F}$ & \\
\hline $\mathrm{H}$ & 43 & $\begin{array}{r}\text { Underliner } \\
\text { Slot Vent } \\
\text { Pipe }\end{array}$ & & 07/03/02 & CCTV & / & 821 & $\begin{array}{l}\text { Inspection was made to } \\
\text { document the condition of the } \\
\text { inner concrete underliner and the } \\
\text { surface of the carbon steel } \\
\text { secondary containment. No } \\
\text { degradation of the concrete liner } \\
\text { was observed. No spalling, } \\
\text { crumbling, exposed rebar, or } \\
\text { stress pattern cracks were } \\
\text { noted. Mild surface corrosion } \\
\text { was observed on the secondary } \\
\text { steel liner. }\end{array}$ \\
\hline $\mathrm{H}$ & 43 & $A-01$ & (A) & 01/09/02 & DP & l & P02081:01-27 & Tank condition was normal. \\
\hline $\mathrm{H}$ & 43 & $\mathrm{~A}-02$ & (A) & 01/09/02 & DP & l & P02082:01-27 & Tank condition was normal. \\
\hline $\mathrm{H}$ & 43 & $\mathrm{~A}-02$ & (A) & 01/09/02 & DP & / & P02082:27 & $\begin{array}{l}\text { The conductivity probe was } \\
\text { deployed within the setpoint } \\
\text { limits. }\end{array}$ \\
\hline $\mathrm{H}$ & 43 & A-03 & (A) & 01/09/02 & DP & / & P02083:01-26 & Tank condition was normal. \\
\hline $\mathrm{H}$ & 43 & A-03 & (A) & 01/09/02 & DP & / & P02083:21 & $\begin{array}{l}\text { The conductivity probe was } \\
\text { deployed within the setpoint } \\
\text { limits. }\end{array}$ \\
\hline $\mathrm{H}$ & 43 & $\mathrm{~A}-04$ & (A) & 01/09/02 & DP & l & P02084:01-28 & $\begin{array}{l}\text { Tank condition was normal. } \\
\text { Stains and marks on the annulus } \\
\text { floor had increased since last } \\
\text { inspected. }\end{array}$ \\
\hline $\mathrm{H}$ & 43 & $\mathrm{~A}-04$ & (A) & 01/09/02 & DP & l & P02084:21 & $\begin{array}{l}\text { The conductivity probe was } \\
\text { deployed within the setpoint } \\
\text { limits. }\end{array}$ \\
\hline $\mathrm{H}$ & 43 & P-01 & (A) & 01/08/02 & WAP & / & P02001:01 & Tank condition was normal. \\
\hline $\mathrm{H}$ & 43 & P-02 & (A) & 01/08/02 & WAP & / & P02001:02 & Tank condition was normal. \\
\hline $\mathrm{H}$ & 43 & P-03 & (A) & 01/08/02 & WAP & I & P02001:03 & Tank condition was normal. \\
\hline $\mathrm{H}$ & 43 & P-04 & (A) & 01/08/02 & WAP & 1 & P02001:04 & Tank condition was normal. \\
\hline $\mathrm{H}$ & 43 & P-05 & (A) & 01/08/02 & WAP & | & P02001:05 & Tank condition was normal. \\
\hline $\mathrm{H}$ & 43 & P-06 & $(\mathrm{A})$ & 01/08/02 & WAP & 1 & P02001:11 & Tank condition was normal. \\
\hline
\end{tabular}




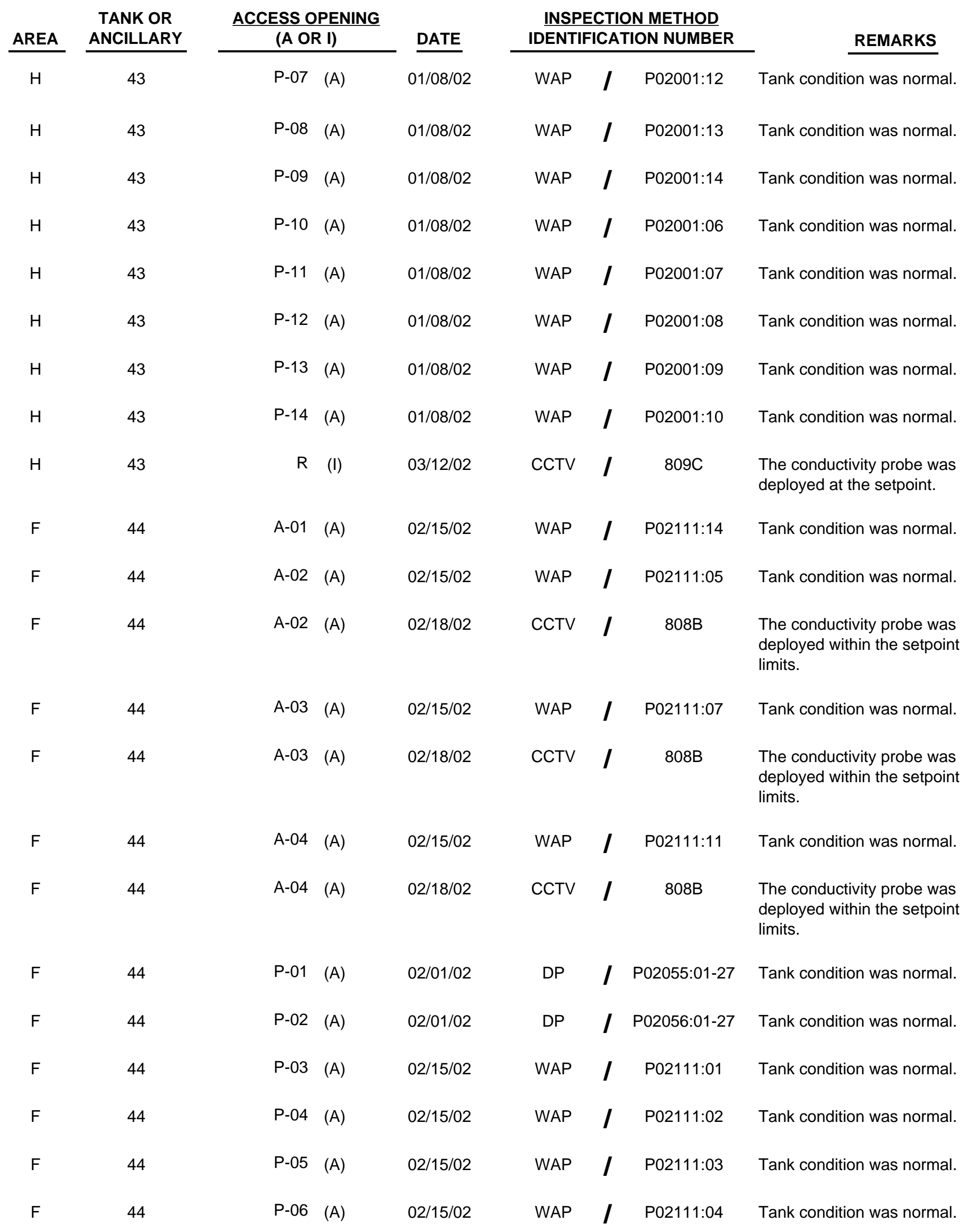




\begin{tabular}{|c|c|c|c|c|c|c|c|c|}
\hline \multirow{2}{*}{$\frac{\text { AREA }}{\mathrm{F}}$} & \multirow{2}{*}{$\begin{array}{c}\text { TANK OR } \\
\text { ANCILLARY } \\
44\end{array}$} & \multicolumn{2}{|c|}{$\frac{\text { ACCESS OPENING }}{(\text { A OR I) }}$} & \multirow{2}{*}{$\frac{\text { DATE }}{02 / 15 / 02}$} & \multicolumn{3}{|c|}{$\begin{array}{l}\text { INSPECTION METHOD } \\
\text { IDENTIFICATION NUMBER }\end{array}$} & \multirow{2}{*}{$\frac{\text { REMARKS }}{\text { Tank condition was normal. }}$} \\
\hline & & P-07 & (A) & & WAP & l & P02111:06 & \\
\hline $\mathrm{F}$ & 44 & P-08 & (A) & 02/01/02 & DP & / & P02057:01-27 & Tank condition was normal. \\
\hline $\mathrm{F}$ & 44 & P-09 & (A) & 02/01/02 & DP & / & P02058:01-27 & Tank condition was normal. \\
\hline $\mathrm{F}$ & 44 & $P-10$ & (A) & 02/15/02 & WAP & / & P02111:08 & Tank condition was normal. \\
\hline $\mathrm{F}$ & 44 & $\mathrm{P}-11$ & (A) & $02 / 15 / 02$ & WAP & l & P02111:09 & Tank condition was normal. \\
\hline $\mathrm{F}$ & 44 & $\mathrm{P}-12$ & (A) & $02 / 15 / 02$ & WAP & / & P02111:10 & Tank condition was normal. \\
\hline $\mathrm{F}$ & 44 & $P-13$ & (A) & $02 / 15 / 02$ & WAP & / & P02111:12 & Tank condition was normal. \\
\hline $\mathrm{F}$ & 44 & $P-14$ & (A) & 02/15/02 & WAP & / & P02111:13 & Tank condition was normal. \\
\hline $\mathrm{F}$ & 44 & G & (I) & 04/24/02 & CCTV & / & $809 \mathrm{~F}$ & $\begin{array}{l}\text { CCTV was used to document the } \\
\text { conditions of the reel tape and } \\
\text { the HLLCP. The reel tape was } \\
\text { covered with salt, and the } \\
\text { HLLCP was contacting the } \\
\text { waste. }\end{array}$ \\
\hline $\mathrm{F}$ & 44 & G & (I) & 04/24/02 & CCTV & / & $809 F$ & $\begin{array}{l}\text { Inspection was made to } \\
\text { document the conditions of the } \\
\text { reel tape and the HLLCP after } \\
\text { flushing. The reel tape was free } \\
\text { of salt and was positioned above } \\
\text { a pool of liquid. The HLLCP } \\
\text { appeared to be contacting the } \\
\text { waste. }\end{array}$ \\
\hline $\mathrm{F}$ & 44 & $\mathrm{G}$ & (I) & 04/26/02 & CCTV & / & $809 \mathrm{~F}$ & $\begin{array}{l}\text { CCTV was used to flush the } \\
\text { HLLCP. The HLLCP was free of } \\
\text { deposits and did not appear to } \\
\text { be contacting the waste. }\end{array}$ \\
\hline $\mathrm{F}$ & 44 & G & (I) & 07/02/02 & CCTV & l & 846 & $\begin{array}{l}\text { Inspection verified that the } \\
\text { HLLCP was properly deployed. }\end{array}$ \\
\hline $\mathrm{F}$ & 44 & $\mathrm{G}$ & (I) & 08/18/02 & CCTV & I & 846 & $\begin{array}{l}\text { Inspection revealed that the } \\
\text { HLLCP was contacting the waste } \\
\text { surface. The HLLCP was } \\
\text { improperly positioned. }\end{array}$ \\
\hline $\mathrm{F}$ & 44 & G & (I) & $10 / 08 / 02$ & CCTV & / & 846 & $\begin{array}{l}\text { Inspection verified that no solids } \\
\text { or obstructions existed beneath } \\
\text { the reel tape or HLLCP. }\end{array}$ \\
\hline
\end{tabular}




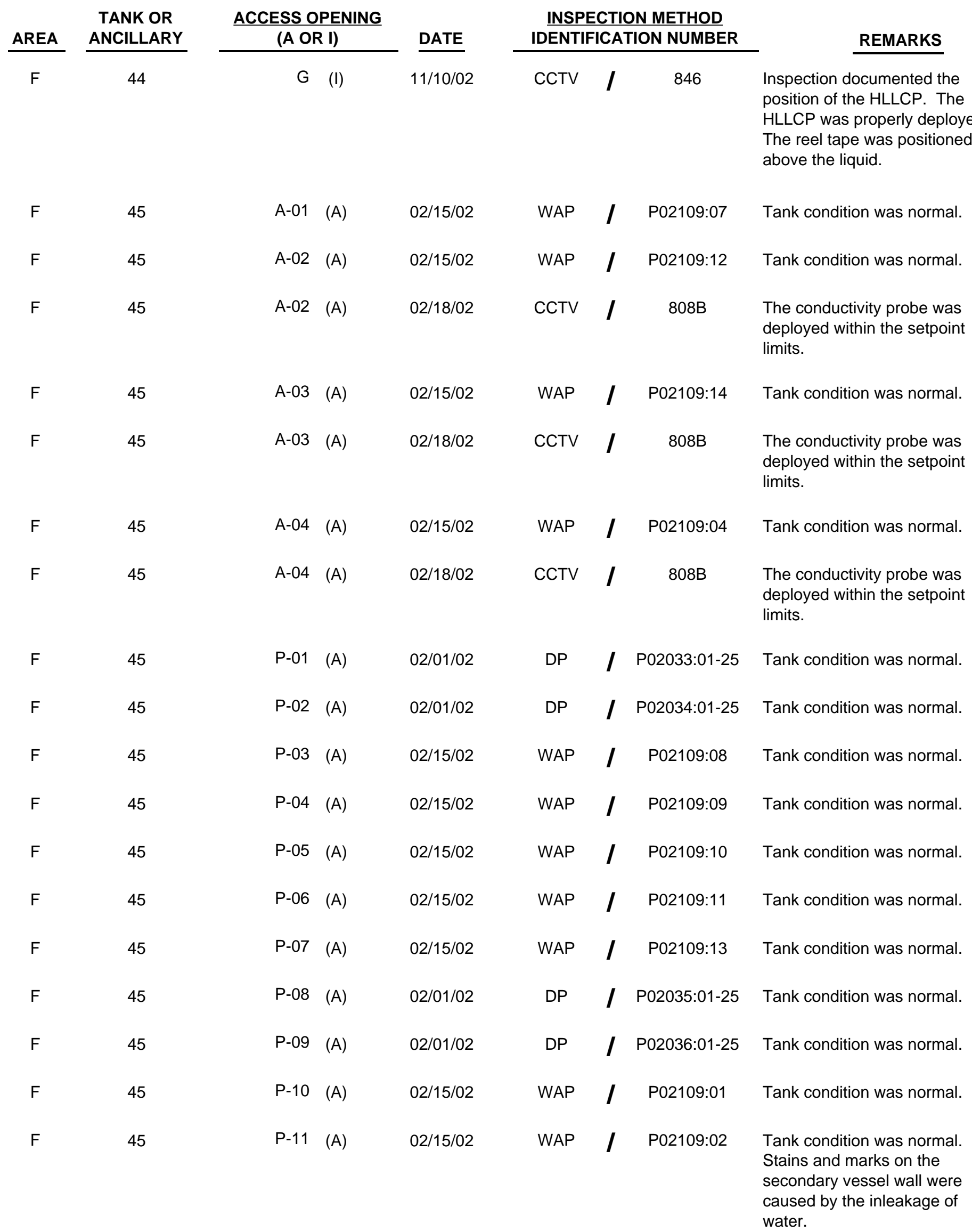




\begin{tabular}{|c|c|c|c|c|c|c|c|c|}
\hline \multirow{2}{*}{$\frac{\text { AREA }}{\mathrm{F}}$} & \multirow{2}{*}{$\begin{array}{c}\text { TANK OR } \\
\text { ANCILLARY } \\
45\end{array}$} & \multicolumn{2}{|c|}{$\frac{\text { ACCESS OPENING }}{(A \text { OR I) }}$} & \multirow{2}{*}{$\frac{\text { DATE }}{02 / 15 / 02}$} & \multicolumn{3}{|c|}{$\begin{array}{l}\text { INSPECTION METHOD } \\
\text { IDENTIFICATION NUMBER }\end{array}$} & \multirow{2}{*}{$\frac{\text { REMARKS }}{\text { Tank condition was normal. }}$} \\
\hline & & P-12 & (A) & & WAP & l & P02109:03 & \\
\hline $\mathrm{F}$ & 45 & $P-13$ & (A) & $02 / 15 / 02$ & WAP & l & P02109:05 & Tank condition was normal. \\
\hline $\mathrm{F}$ & 45 & $P-14$ & (A) & $02 / 15 / 02$ & WAP & / & P02109:06 & Tank condition was normal. \\
\hline $\mathrm{F}$ & 46 & LDB-02 & & 08/30/02 & CCTV & l & 841 & $\begin{array}{l}\text { The conductivity probe was } \\
\text { deployed within the setpoint } \\
\text { limits. }\end{array}$ \\
\hline $\mathrm{F}$ & 46 & $\begin{array}{r}\text { Underliner } \\
\text { Sump }\end{array}$ & & 01/22/02 & CCTV & l & $809 \mathrm{~A}$ & $\begin{array}{l}\text { Inspection revealed corrosion } \\
\text { particles, sand and silt beneath } \\
\text { the conductivity probe standpipe } \\
\text { and the dip tubes. }\end{array}$ \\
\hline $\mathrm{F}$ & 46 & $\begin{array}{r}\text { Underliner } \\
\text { Sump }\end{array}$ & & 06/03/02 & CCTV & l & 807A & $\begin{array}{l}\text { Inspection revealed corrosion } \\
\text { particles, small flakes of tape, } \\
\text { paper and unidentified material } \\
\text { in the underliner sump. }\end{array}$ \\
\hline $\mathrm{F}$ & 46 & $A-01$ & (A) & $02 / 15 / 02$ & WAP & l & P02118:06 & Tank condition was normal. \\
\hline $\mathrm{F}$ & 46 & $\mathrm{~A}-02$ & (A) & 02/07/02 & CCTV & l & 808B & $\begin{array}{l}\text { The conductivity probe was } \\
\text { deployed within the setpoint } \\
\text { limits. }\end{array}$ \\
\hline $\mathrm{F}$ & 46 & A-02 & (A) & $02 / 15 / 02$ & WAP & / & P02118:02 & Tank condition was normal. \\
\hline $\mathrm{F}$ & 46 & A-02 & (A) & 08/01/02 & CCTV & l & 828 & $\begin{array}{l}\text { Inspection revealed water on the } \\
\text { annulus floor and ventilation } \\
\text { duct. The water entered the } \\
\text { annulus via penetration lines } \\
\text { designed WC2 and WC3. }\end{array}$ \\
\hline $\mathrm{F}$ & 46 & A-03 & (A) & $02 / 15 / 02$ & WAP & / & P02118:13 & Tank condition was normal. \\
\hline $\mathrm{F}$ & 46 & A-03 & (A) & 02/19/02 & CCTV & / & $808 B$ & $\begin{array}{l}\text { The conductivity probe was } \\
\text { deployed within the setpoint } \\
\text { limits. }\end{array}$ \\
\hline $\mathrm{F}$ & 46 & A-04 & (A) & $02 / 15 / 02$ & WAP & / & P02118:09 & Tank condition was normal. \\
\hline $\mathrm{F}$ & 46 & A-04 & (A) & 02/19/02 & CCTV & l & 808B & $\begin{array}{l}\text { The conductivity probe was } \\
\text { deployed within the setpoint } \\
\text { limits. }\end{array}$ \\
\hline $\mathrm{F}$ & 46 & P-01 & (A) & 02/01/02 & DP & / & P02037:01-27 & Tank condition was normal. \\
\hline $\mathrm{F}$ & 46 & P-02 & $(\mathrm{A})$ & 02/01/02 & DP & / & P02038:01-25 & Tank condition was normal. \\
\hline $\mathrm{F}$ & 46 & P-03 & (A) & 02/15/02 & WAP & / & P02118:08 & Tank condition was normal. \\
\hline
\end{tabular}




\begin{tabular}{|c|c|c|c|c|c|c|c|c|}
\hline \multirow{2}{*}{$\frac{\text { AREA }}{F}$} & \multirow{2}{*}{$\begin{array}{c}\text { TANK OR } \\
\text { ANCILLARY } \\
46\end{array}$} & \multicolumn{2}{|c|}{$\frac{\text { ACCESS OPENING }}{(A \text { OR I) }}$} & \multirow{2}{*}{$\frac{\text { DATE }}{\text { 02/15/02 }}$} & \multicolumn{3}{|c|}{$\begin{array}{l}\text { INSPECTION METHOD } \\
\text { IDENTIFICATION NUMBER }\end{array}$} & \multirow{2}{*}{$\begin{array}{l}\quad \text { REMARKS } \\
\text { Tank condition was normal. } \\
\text { Stains and marks on the } \\
\text { secondary vessel wall were } \\
\text { caused by the inleakage of } \\
\text { water. }\end{array}$} \\
\hline & & P-04 & $(\mathrm{A})$ & & WAP & l & P02118:10 & \\
\hline $\mathrm{F}$ & 46 & P-05 & $(\mathrm{A})$ & $02 / 15 / 02$ & WAP & / & P02118:11 & Tank condition was normal. \\
\hline $\mathrm{F}$ & 46 & P-06 & $(\mathrm{A})$ & $02 / 15 / 02$ & WAP & l & P02118:12 & Tank condition was normal. \\
\hline $\mathrm{F}$ & 46 & P-07 & (A) & $02 / 15 / 02$ & WAP & / & P02118:14 & Tank condition was normal. \\
\hline $\mathrm{F}$ & 46 & P-08 & $(\mathrm{A})$ & $02 / 01 / 02$ & DP & l & P02039:01-28 & Tank condition was normal. \\
\hline $\mathrm{F}$ & 46 & P-09 & (A) & $02 / 01 / 02$ & DP & l & P02040:01-27 & $\begin{array}{l}\text { Tank condition was normal. } \\
\text { Stains and marks on the } \\
\text { secondary vessel wall were } \\
\text { caused by the inleakage of } \\
\text { water. }\end{array}$ \\
\hline $\mathrm{F}$ & 46 & $P-10$ & (A) & $02 / 15 / 02$ & WAP & / & P02118:01 & Tank condition was normal. \\
\hline $\mathrm{F}$ & 46 & $P-11$ & (A) & 02/15/02 & WAP & / & P02118:03 & Tank condition was normal. \\
\hline $\mathrm{F}$ & 46 & $P-12$ & (A) & 02/15/02 & WAP & / & P02118:04 & $\begin{array}{l}\text { Tank condition was normal. } \\
\text { Stains and marks on the } \\
\text { secondary vessel wall were } \\
\text { caused by the inleakage of } \\
\text { water. }\end{array}$ \\
\hline $\mathrm{F}$ & 46 & $P-13$ & (A) & 02/15/02 & WAP & I & P02118:05 & $\begin{array}{l}\text { Tank condition was normal. } \\
\text { Stains and marks on the } \\
\text { secondary vessel wall were } \\
\text { caused by the inleakage of } \\
\text { water. }\end{array}$ \\
\hline $\mathrm{F}$ & 46 & $\mathrm{P}-14$ & $(\mathrm{~A})$ & 02/15/02 & WAP & & P02118:07 & Tank condition was normal. \\
\hline $\mathrm{F}$ & 46 & $\mathrm{H}$ & (I) & $01 / 17 / 02$ & CCTV & 7 & 809 & $\begin{array}{l}\text { Inspection revealed that a salt } \\
\text { crust layer had formed on the } \\
\text { waste surface. Liquid was } \\
\text { visible through depressions in } \\
\text { the salt crust, particularly } \\
\text { beneath the riser openings. A } \\
\text { thin layer of salt had formed on } \\
\text { the tank wall and center column. }\end{array}$ \\
\hline
\end{tabular}




\begin{tabular}{|c|c|c|c|c|c|c|c|c|}
\hline \multirow{2}{*}{$\frac{\text { AREA }}{F}$} & \multirow{2}{*}{$\begin{array}{c}\begin{array}{c}\text { TANK OR } \\
\text { ANCILLARY }\end{array} \\
46\end{array}$} & \multicolumn{2}{|c|}{$\frac{\text { ACCESS OPENING }}{(A \text { OR I) }}$} & \multirow{2}{*}{$\frac{\text { DATE }}{05 / 15 / 02}$} & \multicolumn{3}{|c|}{$\begin{array}{l}\text { INSPECTION METHOD } \\
\text { IDENTIFICATION NUMBER }\end{array}$} & \multirow{2}{*}{$\begin{array}{l}\text { REMARKS } \\
\text { Inspection was made to } \\
\text { document conditions beneath the } \\
\text { C1 riser. The jet and surface } \\
\text { area beneath the riser were not } \\
\text { visible. The cooling coils were } \\
\text { encrusted with approximately } 10 \\
\text { inches of salt. }\end{array}$} \\
\hline & & $\mathrm{H}$ & (I) & & CCTV & / & 828 & \\
\hline $\mathrm{F}$ & 47 & $A-01$ & $(\mathrm{~A})$ & $02 / 15 / 02$ & WAP & / & P02110:02 & Tank condition was normal. \\
\hline $\mathrm{F}$ & 47 & A-02 & $(\mathrm{A})$ & $02 / 15 / 02$ & WAP & / & P02110:14 & Tank condition was normal. \\
\hline $\mathrm{F}$ & 47 & A-02 & (A) & 02/19/02 & CCTV & / & 808B & $\begin{array}{l}\text { The conductivity probe was } \\
\text { deployed within the setpoint } \\
\text { limits. }\end{array}$ \\
\hline $\mathrm{F}$ & 47 & A-03 & $(\mathrm{A})$ & $02 / 15 / 02$ & WAP & l & P02110:12 & Tank condition was normal. \\
\hline $\mathrm{F}$ & 47 & A-03 & (A) & 02/19/02 & CCTV & l & 808B & $\begin{array}{l}\text { The conductivity probe was } \\
\text { deployed within the setpoint } \\
\text { limits. }\end{array}$ \\
\hline $\mathrm{F}$ & 47 & A-04 & (A) & $02 / 15 / 02$ & WAP & / & P02110:06 & Tank condition was normal. \\
\hline $\mathrm{F}$ & 47 & A-04 & (A) & 02/19/02 & CCTV & / & 808B & $\begin{array}{l}\text { The conductivity probe was } \\
\text { deployed within the setpoint } \\
\text { limits. }\end{array}$ \\
\hline $\mathrm{F}$ & 47 & P-01 & (A) & 02/01/02 & DP & / & P02029:01-27 & Tank condition was normal. \\
\hline $\mathrm{F}$ & 47 & P-02 & (A) & 02/01/02 & DP & / & P02030:01-27 & Tank condition was normal. \\
\hline $\mathrm{F}$ & 47 & P-03 & $(\mathrm{A})$ & $02 / 15 / 02$ & WAP & / & P02110:08 & Tank condition was normal. \\
\hline $\mathrm{F}$ & 47 & P-04 & $(\mathrm{A})$ & $02 / 15 / 02$ & WAP & / & P02110:09 & Tank condition was normal. \\
\hline $\mathrm{F}$ & 47 & P-05 & $(\mathrm{A})$ & $02 / 15 / 02$ & WAP & / & P02110:10 & Tank condition was normal. \\
\hline $\mathrm{F}$ & 47 & P-06 & $(\mathrm{A})$ & $02 / 15 / 02$ & WAP & / & P02110:11 & Tank condition was normal. \\
\hline $\mathrm{F}$ & 47 & P-07 & $(\mathrm{A})$ & $02 / 15 / 02$ & WAP & / & P02110:13 & $\begin{array}{l}\text { Tank condition was normal. A } \\
\text { masselin cloth was observed on } \\
\text { the annulus floor. }\end{array}$ \\
\hline $\mathrm{F}$ & 47 & P-08 & $(\mathrm{A})$ & $02 / 01 / 02$ & DP & / & P02031:01-27 & Tank condition was normal. \\
\hline $\mathrm{F}$ & 47 & P-09 & $(\mathrm{A})$ & 02/01/02 & DP & / & P02032:01-27 & Tank condition was normal. \\
\hline $\mathrm{F}$ & 47 & P-10 & (A) & $02 / 15 / 02$ & WAP & / & P02110:07 & Tank condition was normal. \\
\hline $\mathrm{F}$ & 47 & $P-11$ & $(\mathrm{~A})$ & 02/15/02 & WAP & 1 & P02110:01 & Tank condition was normal. \\
\hline
\end{tabular}




\begin{tabular}{|c|c|c|c|c|c|c|c|}
\hline \multirow{2}{*}{$\frac{\text { AREA }}{\mathrm{F}}$} & \multirow{2}{*}{$\begin{array}{c}\text { TANK OR } \\
\text { ANCILLARY } \\
47\end{array}$} & $\frac{\text { ACCESS OPENING }}{(\text { A OR I) }}$ & \multirow{2}{*}{$\frac{\text { DATE }}{02 / 15 / 02}$} & \multicolumn{3}{|c|}{ INSPECTION METHOD } & REMARKS \\
\hline & & $\mathrm{P}-12 \quad(\mathrm{~A})$ & & WAP & / & P02110:03 & Tank condition was normal. \\
\hline $\mathrm{F}$ & 47 & $P-13 \quad(A)$ & $02 / 15 / 02$ & WAP & / & P02110:04 & Tank condition was normal. \\
\hline $\mathrm{F}$ & 47 & $\mathrm{P}-14 \quad(\mathrm{~A})$ & $02 / 15 / 02$ & WAP & / & P02110:05 & Tank condition was normal. \\
\hline $\mathrm{H}$ & 48 & LDB-02 & $08 / 11 / 02$ & CCTV & / & 841 & $\begin{array}{l}\text { CCTV was used to remove an } \\
\text { abandoned conductivity probe. } \\
\text { A new conductivity probe was } \\
\text { deployed within the setpoint } \\
\text { limits. }\end{array}$ \\
\hline $\mathrm{H}$ & 48 & LDB-03 & 07/31/02 & CCTV & / & 841 & $\begin{array}{l}\text { The conductivity probe was } \\
\text { deployed within the setpoint } \\
\text { limits. }\end{array}$ \\
\hline $\mathrm{H}$ & 48 & LDB-04 & 06/18/02 & CCTV & l & 841 & $\begin{array}{l}\text { The conductivity probe was } \\
\text { deployed within the setpoint } \\
\text { limits. }\end{array}$ \\
\hline $\mathrm{H}$ & 48 & LDB-04 & $11 / 24 / 02$ & CCTV & / & 841 & $\begin{array}{l}\text { The conductivity probe was } \\
\text { deployed within the setpoint } \\
\text { limits. }\end{array}$ \\
\hline $\mathrm{H}$ & 48 & LDB-05 & $11 / 24 / 02$ & CCTV & / & 841 & $\begin{array}{l}\text { The conductivity probe was } \\
\text { deployed within the setpoint } \\
\text { limits. }\end{array}$ \\
\hline $\mathrm{H}$ & 48 & LDB-07 & 07/27/02 & CCTV & I & 841 & $\begin{array}{l}\text { The conductivity probe was } \\
\text { deployed within the setpoint } \\
\text { limits. }\end{array}$ \\
\hline $\mathrm{H}$ & 48 & LDB-08 & 07/27/02 & CCTV & I & 841 & $\begin{array}{l}\text { The conductivity probe was } \\
\text { deployed within the setpoint } \\
\text { limits. }\end{array}$ \\
\hline $\mathrm{H}$ & 48 & LDB-09 & 07/28/02 & CCTV & I & 841 & $\begin{array}{l}\text { The conductivity probe was } \\
\text { deployed within the setpoint } \\
\text { limits. }\end{array}$ \\
\hline $\mathrm{H}$ & 48 & LDB-10 & $08 / 11 / 02$ & CCTV & I & 841 & $\begin{array}{l}\text { CCTV was used to flush the } \\
\text { LDB. The conductivity probe } \\
\text { was redeployed within the } \\
\text { setpoint limits. }\end{array}$ \\
\hline $\mathrm{H}$ & 48 & LDB-12 & 08/07/02 & CCTV & I & 841 & $\begin{array}{l}\text { The conductivity probe was } \\
\text { deployed within the setpoint } \\
\text { limits. }\end{array}$ \\
\hline $\mathrm{H}$ & 48 & LDB-13 & 05/28/02 & CCTV & I & $807 A$ & $\begin{array}{l}\text { The conductivity probe could not } \\
\text { be properly deployed due to } \\
\text { loose corrosion particles beneath } \\
\text { the standpipe. }\end{array}$ \\
\hline
\end{tabular}




\begin{tabular}{|c|c|c|c|c|c|c|c|c|}
\hline \multirow{2}{*}{$\frac{\text { AREA }}{\mathrm{H}}$} & \multirow{2}{*}{$\begin{array}{c}\text { TANK OR } \\
\text { ANCILLARY } \\
48\end{array}$} & \multicolumn{2}{|c|}{$\frac{\text { ACCESS OPENING }}{(A \text { OR I) }}$} & \multirow{2}{*}{$\frac{\text { DATE }}{05 / 30 / 02}$} & \multicolumn{3}{|c|}{$\begin{array}{l}\text { INSPECTION METHOD } \\
\text { IDENTIFICATION NUMBER }\end{array}$} & \multirow{2}{*}{$\begin{array}{l}\text { REMARKS } \\
\text { The conductivity probe was } \\
\text { deployed within the setpoint } \\
\text { limits. }\end{array}$} \\
\hline & & LDB-13 & & & CCTV & l & $807 \mathrm{~A}$ & \\
\hline $\mathrm{H}$ & 48 & $A-01$ & $(\mathrm{~A})$ & $10 / 23 / 02$ & WAP & l & P02232:07 & Tank condition was normal. \\
\hline $\mathrm{H}$ & 48 & A-02 & (A) & 01/09/02 & CCTV & / & 808 & $\begin{array}{l}\text { The conductivity probe was } \\
\text { deployed within the setpoint } \\
\text { limits. }\end{array}$ \\
\hline $\mathrm{H}$ & 48 & A-02 & (A) & $10 / 23 / 02$ & WAP & l & P02232:04 & Tank condition was normal. \\
\hline $\mathrm{H}$ & 48 & A-03 & $(\mathrm{A})$ & 01/09/02 & CCTV & l & 808 & $\begin{array}{l}\text { The conductivity probe was } \\
\text { deployed within the setpoint } \\
\text { limits. }\end{array}$ \\
\hline $\mathrm{H}$ & 48 & A-03 & $(\mathrm{A})$ & $10 / 23 / 02$ & WAP & I & P02232:02 & Tank condition was normal. \\
\hline $\mathrm{H}$ & 48 & A-04 & $(\mathrm{A})$ & 01/09/02 & CCTV & l & 808 & $\begin{array}{l}\text { The conductivity probe was } \\
\text { deployed within the setpoint } \\
\text { limits. }\end{array}$ \\
\hline $\mathrm{H}$ & 48 & A-04 & $(\mathrm{A})$ & $10 / 23 / 02$ & WAP & l & P02232:11 & Tank condition was normal. \\
\hline $\mathrm{H}$ & 48 & P-01 & $(A)$ & 09/20/02 & DP & l & P02214:01-28 & Tank condition was normal. \\
\hline $\mathrm{H}$ & 48 & P-02 & $(A)$ & 09/20/02 & DP & l & P02212:01-27 & Tank condition was normal. \\
\hline $\mathrm{H}$ & 48 & P-03 & $(\mathrm{A})$ & $10 / 23 / 02$ & WAP & l & P02232:08 & Tank condition was normal. \\
\hline $\mathrm{H}$ & 48 & P-04 & $(\mathrm{A})$ & $10 / 23 / 02$ & WAP & / & P02232:05 & Tank condition was normal. \\
\hline $\mathrm{H}$ & 48 & P-05 & (A) & $10 / 23 / 02$ & WAP & / & P02232:03 & Tank condition was normal. \\
\hline $\mathrm{H}$ & 48 & P-06 & (A) & 09/20/02 & DP & / & P02211:01-27 & Tank condition was normal. \\
\hline $\mathrm{H}$ & 48 & P-07 & $(\mathrm{A})$ & 09/20/02 & DP & / & P02209:01-27 & $\begin{array}{l}\text { Tank condition was normal. } \\
\text { Stains on the primary vessel wall } \\
\text { were due to inleakage of water. }\end{array}$ \\
\hline $\mathrm{H}$ & 48 & P-08 & $(A)$ & 09/20/02 & DP & I & P02213:01-27 & Tank condition was normal. \\
\hline $\mathrm{H}$ & 48 & P-09 & $(A)$ & 09/20/02 & DP & / & P02210:01-27 & Tank condition was normal. \\
\hline $\mathrm{H}$ & 48 & $P-10$ & $(\mathrm{~A})$ & $10 / 23 / 02$ & WAP & / & $P 02232: 10$ & Tank condition was normal. \\
\hline $\mathrm{H}$ & 48 & $P-11$ & $(\mathrm{~A})$ & $10 / 23 / 02$ & WAP & 1 & P02232:01 & Tank condition was normal. \\
\hline $\mathrm{H}$ & 48 & $P-13$ & $(\mathrm{~A})$ & $10 / 23 / 02$ & WAP & 7 & P02232:09 & Tank condition was normal. \\
\hline $\mathrm{H}$ & 48 & P-14 & $(\mathrm{A})$ & $10 / 23 / 02$ & WAP & l & P02232:06 & Tank condition was normal. \\
\hline
\end{tabular}




\begin{tabular}{|c|c|c|c|c|c|c|c|c|}
\hline \multirow{2}{*}{$\frac{\text { AREA }}{\mathrm{H}}$} & \multirow{2}{*}{$\begin{array}{c}\text { TANK OR } \\
\text { ANCILLARY } \\
49\end{array}$} & \multicolumn{2}{|c|}{$\frac{\text { ACCESS OPENING }}{(\text { A OR I) }}$} & \multirow{2}{*}{$\frac{\text { DATE }}{09 / 24 / 02}$} & \multicolumn{3}{|c|}{$\begin{array}{l}\text { INSPECTION METHOD } \\
\text { IDENTIFICATION NUMBER }\end{array}$} & \multirow{2}{*}{$\begin{array}{l}\text { REMARKS } \\
\text { The conductivity probe was } \\
\text { deployed within the setpoint } \\
\text { limits. }\end{array}$} \\
\hline & & LDB-01 & & & CCTV & / & 861 & \\
\hline $\mathrm{H}$ & 49 & A-01 & (A) & $10 / 22 / 02$ & WAP & / & P02233:08 & Tank condition was normal. \\
\hline $\mathrm{H}$ & 49 & A-02 & (A) & 01/09/02 & CCTV & / & 808 & $\begin{array}{l}\text { The conductivity probe was } \\
\text { deployed within the setpoint } \\
\text { limits. }\end{array}$ \\
\hline $\mathrm{H}$ & 49 & A-02 & (A) & $10 / 22 / 02$ & WAP & / & P02233:05 & Tank condition was normal. \\
\hline $\mathrm{H}$ & 49 & A-03 & (A) & 01/09/02 & CCTV & / & 808 & $\begin{array}{l}\text { The conductivity probe was } \\
\text { deployed within the setpoint } \\
\text { limits. }\end{array}$ \\
\hline $\mathrm{H}$ & 49 & A-03 & (A) & $10 / 22 / 02$ & WAP & / & P02233:01 & Tank condition was normal. \\
\hline $\mathrm{H}$ & 49 & A-04 & (A) & 01/09/02 & CCTV & / & 808 & $\begin{array}{l}\text { The conductivity probe was } \\
\text { deployed within the setpoint } \\
\text { limits. }\end{array}$ \\
\hline $\mathrm{H}$ & 49 & A-04 & (A) & $10 / 22 / 02$ & WAP & / & P02233:12 & Tank condition was normal. \\
\hline $\mathrm{H}$ & 49 & P-01 & (A) & 09/05/02 & DP & / & P02222:01-27 & Tank condition was normal. \\
\hline $\mathrm{H}$ & 49 & P-02 & (A) & 09/05/02 & DP & l & P02219:01-26 & Tank condition was normal. \\
\hline $\mathrm{H}$ & 49 & P-03 & (A) & $10 / 22 / 02$ & WAP & l & P02233:07 & $\begin{array}{l}\text { Tank condition was normal. A } \\
\text { masselin cloth observed on the } \\
\text { annulus floor beneath the riser } \\
\text { had been relocated due to } \\
\text { annulus ventilation. }\end{array}$ \\
\hline $\mathrm{H}$ & 49 & P-04 & (A) & $10 / 22 / 02$ & WAP & / & P02233:06 & Tank condition was normal. \\
\hline $\mathrm{H}$ & 49 & P-05 & (A) & $10 / 22 / 02$ & WAP & / & P02233:04 & Tank condition was normal. \\
\hline $\mathrm{H}$ & 49 & P-06 & (A) & $10 / 22 / 02$ & WAP & / & P02233:03 & Tank condition was normal. \\
\hline $\mathrm{H}$ & 49 & P-07 & (A) & $10 / 22 / 02$ & WAP & / & P02233:02 & Tank condition was normal. \\
\hline $\mathrm{H}$ & 49 & P-08 & (A) & 09/05/02 & $\mathrm{DP}$ & / & P02221:01-26 & Tank condition was normal. \\
\hline $\mathrm{H}$ & 49 & P-09 & (A) & 09/05/02 & DP & / & P02220:01-27 & Tank condition was normal. \\
\hline $\mathrm{H}$ & 49 & $P-10$ & (A) & $10 / 22 / 02$ & WAP & / & P02233:14 & Tank condition was normal. \\
\hline $\mathrm{H}$ & 49 & $P-11$ & (A) & $10 / 22 / 02$ & WAP & / & P02233:13 & Tank condition was normal. \\
\hline $\mathrm{H}$ & 49 & $P-12$ & (A) & $10 / 22 / 02$ & WAP & / & P02233:11 & Tank condition was normal. \\
\hline
\end{tabular}




\begin{tabular}{|c|c|c|c|c|c|c|c|c|}
\hline \multirow{2}{*}{$\frac{\text { AREA }}{\mathrm{H}}$} & \multirow{2}{*}{$\begin{array}{c}\text { TANK OR } \\
\text { ANCILLARY } \\
49\end{array}$} & \multicolumn{2}{|c|}{$\frac{\text { ACCESS OPENING }}{(A \text { OR I) }}$} & \multirow{2}{*}{$\frac{\text { DATE }}{10 / 22 / 02}$} & \multicolumn{3}{|c|}{$\begin{array}{l}\text { INSPECTION METHOD } \\
\text { IDENTIFICATION NUMBER }\end{array}$} & \multirow{2}{*}{$\frac{\text { REMARKS }}{\text { Tank condition was normal. }}$} \\
\hline & & $P-13$ & (A) & & WAP & / & P02233:10 & \\
\hline $\mathrm{H}$ & 49 & P-14 & (A) & $10 / 22 / 02$ & WAP & l & P02233:09 & Tank condition was normal. \\
\hline $\mathrm{H}$ & 49 & B-05 & (I) & 05/08/02 & CCTV & / & $809 \mathrm{~F}$ & $\begin{array}{l}\text { Inspection documented the } \\
\text { position of the conductivity } \\
\text { probe. The probe is resting on } \\
\text { the ledge below the connector. }\end{array}$ \\
\hline $\mathrm{H}$ & 50 & LDB-01 & & 03/06/02 & CCTV & / & $807 \mathrm{~A}$ & $\begin{array}{l}\text { The conductivity probe was } \\
\text { deployed within the setpoint } \\
\text { limits. }\end{array}$ \\
\hline $\mathrm{H}$ & 50 & LDB-02 & & 09/24/02 & CCTV & / & 855 & $\begin{array}{l}\text { The conductivity probe was } \\
\text { deployed within the setpoint } \\
\text { limits. }\end{array}$ \\
\hline $\mathrm{H}$ & 50 & LDB-03 & & 03/06/02 & CCTV & / & $807 \mathrm{~A}$ & $\begin{array}{l}\text { The conductivity probe was not } \\
\text { visible due to corrosion particles } \\
\text { and mud beneath the } \\
\text { conductivity probe standpipe. }\end{array}$ \\
\hline $\mathrm{H}$ & 50 & LDB-04 & & $10 / 10 / 02$ & CCTV & l & 841 & $\begin{array}{l}\text { Inspection revealed that the LDB } \\
\text { is clean and that the conductivity } \\
\text { probe was properly positioned. }\end{array}$ \\
\hline $\mathrm{H}$ & 50 & VB & & 04/07/02 & CCTV & / & 809D & $\begin{array}{l}\text { CCTV was used to leak check } \\
\text { Gra-Loc connector. No leaks } \\
\text { were observed. }\end{array}$ \\
\hline $\mathrm{H}$ & 50 & $A-01$ & (A) & $10 / 24 / 02$ & WAP & l & P02231:08 & Tank condition was normal. \\
\hline $\mathrm{H}$ & 50 & A-02 & (A) & 01/09/02 & CCTV & / & 808 & $\begin{array}{l}\text { The conductivity probe was } \\
\text { deployed within the setpoint } \\
\text { limits. }\end{array}$ \\
\hline $\mathrm{H}$ & 50 & A-02 & (A) & $10 / 24 / 02$ & WAP & 1 & P02231:05 & Tank condition was normal. \\
\hline $\mathrm{H}$ & 50 & A-03 & $(\mathrm{A})$ & 01/09/02 & CCTV & / & 808 & $\begin{array}{l}\text { The conductivity probe was } \\
\text { deployed within the setpoint } \\
\text { limits. }\end{array}$ \\
\hline $\mathrm{H}$ & 50 & A-03 & $(\mathrm{A})$ & $10 / 24 / 02$ & WAP & I & P02231:01 & Tank condition was normal. \\
\hline $\mathrm{H}$ & 50 & A-04 & $(\mathrm{A})$ & 01/09/02 & CCTV & l & 808 & $\begin{array}{l}\text { The conductivity probe was } \\
\text { deployed within the setpoint } \\
\text { limits. }\end{array}$ \\
\hline $\mathrm{H}$ & 50 & $A-04$ & (A) & $10 / 24 / 02$ & WAP & 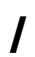 & P02231:12 & Tank condition was normal. \\
\hline $\mathrm{H}$ & 50 & P-01 & (A) & 09/19/02 & $\mathrm{DP}$ & l & P02215:01-29 & Tank condition was normal. \\
\hline $\mathrm{H}$ & 50 & P-02 & $(\mathrm{A})$ & 09/19/02 & $\mathrm{DP}$ & / & P02218:01-27 & Tank condition was normal. \\
\hline
\end{tabular}




\begin{tabular}{|c|c|c|c|c|c|c|c|c|}
\hline \multirow{2}{*}{ AREA } & \multirow{2}{*}{$\begin{array}{c}\text { TANK OR } \\
\text { ANCILLARY } \\
50\end{array}$} & \multicolumn{2}{|c|}{$\frac{\text { ACCESS OPENING }}{(\text { A OR I) }}$} & \multirow{2}{*}{$\frac{\text { DATE }}{10 / 24 / 02}$} & \multicolumn{3}{|c|}{$\begin{array}{l}\text { INSPECTION METHOD } \\
\text { IDENTIFICATION NUMBER }\end{array}$} & \multirow{2}{*}{$\frac{\text { REMARKS }}{\text { Tank condition was normal. }}$} \\
\hline & & P-03 & (A) & & WAP & / & P02231:07 & \\
\hline $\mathrm{H}$ & 50 & P-04 & (A) & $10 / 24 / 02$ & WAP & / & P02231:06 & Tank condition was normal. \\
\hline $\mathrm{H}$ & 50 & P-05 & (A) & $10 / 24 / 02$ & WAP & / & P02231:04 & Tank condition was normal. \\
\hline $\mathrm{H}$ & 50 & P-06 & (A) & $10 / 24 / 02$ & WAP & / & P02231:03 & Tank condition was normal. \\
\hline $\mathrm{H}$ & 50 & P-07 & (A) & $10 / 24 / 02$ & WAP & / & P02231:02 & Tank condition was normal. \\
\hline $\mathrm{H}$ & 50 & P-08 & (A) & 09/19/02 & DP & / & P02217:01-27 & Tank condition was normal. \\
\hline $\mathrm{H}$ & 50 & P-09 & (A) & $12 / 09 / 02$ & DP & / & P02243:01-27 & Tank condition was normal. \\
\hline $\mathrm{H}$ & 50 & P-10 & (A) & $10 / 24 / 02$ & WAP & / & P02231:14 & Tank condition was normal. \\
\hline $\mathrm{H}$ & 50 & $P-11$ & (A) & $10 / 24 / 02$ & WAP & / & P02231:13 & Tank condition was normal. \\
\hline $\mathrm{H}$ & 50 & P-12 & (A) & $10 / 24 / 02$ & WAP & / & P02231:11 & Tank condition was normal. \\
\hline $\mathrm{H}$ & 50 & P-13 & (A) & $10 / 24 / 02$ & WAP & / & P02231:10 & Tank condition was normal. \\
\hline $\mathrm{H}$ & 50 & $\mathrm{P}-14$ & (A) & $10 / 24 / 02$ & WAP & / & P02231:09 & Tank condition was normal. \\
\hline $\mathrm{H}$ & 50 & G & (I) & $10 / 21 / 02$ & CCTV & / & 855 & $\begin{array}{l}\text { CCTV was used to investigate } \\
\text { material balance discrepancies. } \\
\text { A mound was observed beneath } \\
\text { the B5 riser around the transfer } \\
\text { pump. The mound was several } \\
\text { feet above the liquid. }\end{array}$ \\
\hline $\mathrm{H}$ & 50 & G & (I) & $10 / 22 / 02$ & CCTV & / & 855 & $\begin{array}{l}\text { CCTV was used to investigate } \\
\text { material balance discrepancies. } \\
\text { A large mound of solids was } \\
\text { observed beneath the B5 riser } \\
\text { around the transfer pump. The } \\
\text { solids were several feet above } \\
\text { the liquid level. The reel tape } \\
\text { was not observed. }\end{array}$ \\
\hline $\mathrm{H}$ & 50 & $\mathrm{H}$ & (I) & $10 / 22 / 02$ & CCTV & I & 855 & $\begin{array}{l}\text { CCTV was used to investigate } \\
\text { material balance discrepancies. } \\
\text { A mound of solids was observed } \\
\text { beneath the C3 riser. The mound } \\
\text { was several feet above the } \\
\text { liquid. }\end{array}$ \\
\hline $\mathrm{H}$ & 51 & LDB-01 & & $06 / 21 / 02$ & CCTV & I & 841 & $\begin{array}{l}\text { The conductivity probe could not } \\
\text { be properly deployed due to mud } \\
\text { and liquid located beneath the } \\
\text { standpipe. }\end{array}$ \\
\hline
\end{tabular}




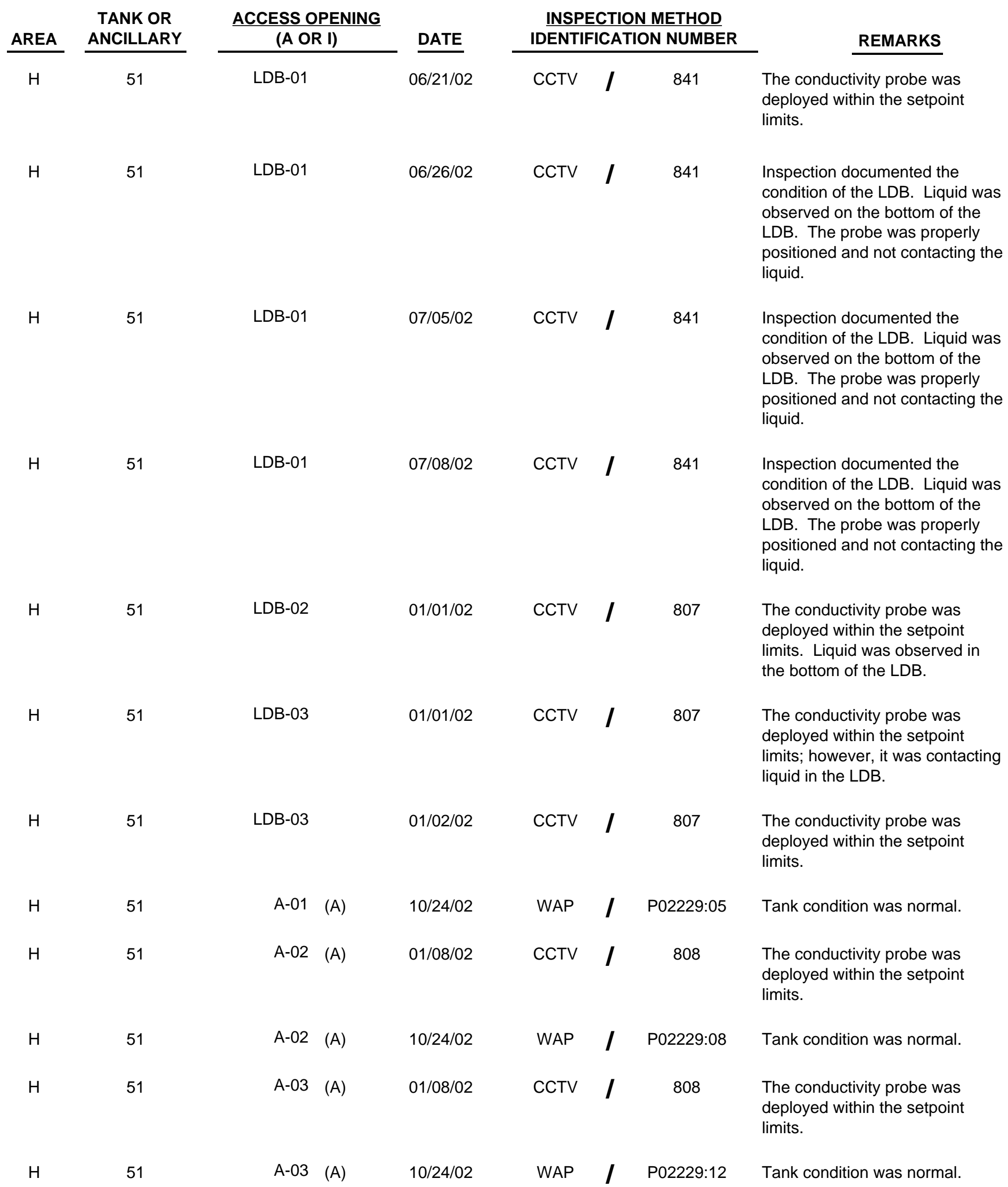




\begin{tabular}{|c|c|c|c|c|c|c|c|c|}
\hline \multirow{2}{*}{$\frac{\text { AREA }}{\mathrm{H}}$} & \multirow{2}{*}{$\begin{array}{c}\text { TANK OR } \\
\text { ANCILLARY } \\
51\end{array}$} & \multicolumn{2}{|c|}{$\frac{\text { ACCESS OPENING }}{(\text { A OR I) }}$} & \multirow{2}{*}{$\frac{\text { DATE }}{01 / 08 / 02}$} & \multicolumn{3}{|c|}{$\begin{array}{l}\text { INSPECTION METHOD } \\
\text { IDENTIFICATION NUMBER }\end{array}$} & \multirow{2}{*}{$\begin{array}{l}\text { REMARKS } \\
\text { The conductivity probe was } \\
\text { deployed within the setpoint } \\
\text { limits. }\end{array}$} \\
\hline & & A-04 & (A) & & CCTV & l & 808 & \\
\hline $\mathrm{H}$ & 51 & A-04 & (A) & $10 / 24 / 02$ & WAP & l & P02229:01 & Tank condition was normal. \\
\hline $\mathrm{H}$ & 51 & P-01 & (A) & 08/26/02 & DP & / & P02192:01-27 & Tank condition was normal. \\
\hline $\mathrm{H}$ & 51 & P-02 & (A) & 08/26/02 & DP & l & P02190:01-27 & Tank condition was normal. \\
\hline $\mathrm{H}$ & 51 & P-03 & (A) & $10 / 24 / 02$ & WAP & l & P02229:06 & Tank condition was normal. \\
\hline $\mathrm{H}$ & 51 & P-04 & (A) & $10 / 24 / 02$ & WAP & l & P02229:07 & Tank condition was normal. \\
\hline $\mathrm{H}$ & 51 & P-05 & (A) & $10 / 24 / 02$ & WAP & l & P02229:09 & Tank condition was normal. \\
\hline $\mathrm{H}$ & 51 & P-06 & (A) & $10 / 24 / 02$ & WAP & l & P02229:10 & Tank condition was normal. \\
\hline $\mathrm{H}$ & 51 & P-07 & (A) & $10 / 24 / 02$ & WAP & / & P02229:11 & Tank condition was normal. \\
\hline $\mathrm{H}$ & 51 & P-08 & (A) & 08/26/02 & $\mathrm{DP}$ & / & P02191:01-25 & Tank condition was normal. \\
\hline $\mathrm{H}$ & 51 & P-09 & $(\mathrm{A})$ & 08/26/02 & DP & l & P02189:01-26 & $\begin{array}{l}\text { Tank condition was normal. The } \\
\text { inleakage of water had washed } \\
\text { away chromate deposits from } \\
\text { the top of the primary vessel wall } \\
\text { to below the bottom knuckle } \\
\text { weld. }\end{array}$ \\
\hline $\mathrm{H}$ & 51 & $P-10$ & (A) & $10 / 24 / 02$ & WAP & l & P02229:13 & Tank condition was normal. \\
\hline $\mathrm{H}$ & 51 & $P-11$ & (A) & $10 / 24 / 02$ & WAP & l & P02229:14 & Tank condition was normal. \\
\hline $\mathrm{H}$ & 51 & $\mathrm{P}-12$ & (A) & $10 / 24 / 02$ & WAP & l & P02229:02 & Tank condition was normal. \\
\hline $\mathrm{H}$ & 51 & $P-13$ & (A) & $10 / 24 / 02$ & WAP & l & P02229:03 & Tank condition was normal. \\
\hline $\mathrm{H}$ & 51 & $\mathrm{P}-14$ & $(\mathrm{~A})$ & $10 / 24 / 02$ & WAP & l & P02229:04 & Tank condition was normal. \\
\hline $\mathrm{H}$ & 51 & B-05 & (l) & 09/25/02 & CCTV & l & 839 & $\begin{array}{l}\text { CCTV was used to document the } \\
\text { position of the conductivity probe } \\
\text { in the spray chamber. The } \\
\text { probe cables extended beyond } \\
\text { the spray chamber into the } \\
\text { primary vessel, but the probe } \\
\text { was not visible. }\end{array}$ \\
\hline $\mathrm{H}$ & 51 & $\mathrm{E}-01$ & (I) & 06/13/02 & CCTV & l & 839 & $\begin{array}{l}\text { Inspection revealed water } \\
\text { entering the tank via the } \mathrm{V} 1 \text { riser. }\end{array}$ \\
\hline
\end{tabular}




\begin{tabular}{|c|c|c|c|c|c|c|c|}
\hline \multirow{2}{*}{$\frac{\text { AREA }}{\mathrm{H}}$} & \multirow{2}{*}{$\begin{array}{c}\text { TANK OR } \\
\text { ANCILLARY } \\
51\end{array}$} & $\frac{\text { ACCESS OPENING }}{(A \text { OR I) }}$ & \multirow{2}{*}{$\frac{\text { DATE }}{06 / 15 / 02}$} & \multicolumn{3}{|c|}{$\begin{array}{l}\text { INSPECTION METHOD } \\
\text { IDENTIFICATION NUMBER }\end{array}$} & \multirow{2}{*}{$\begin{array}{l}\quad \text { REMARKS } \\
\text { CCTV was used to determine the } \\
\text { origin of water observed entering } \\
\text { the tank on } 6 / 13 / 02 \text {. Water was } \\
\text { observed leaking from valve } \\
\text { WTS-V-286. }\end{array}$} \\
\hline & & V-01 (I) & & CCTV & / & 839 & \\
\hline $\mathrm{H}$ & CTS & LDB-04 & 03/26/02 & CCTV & I & $807 A$ & $\begin{array}{l}\text { The conductivity probe was } \\
\text { deployed within the setpoint } \\
\text { limits. }\end{array}$ \\
\hline $\mathrm{F}$ & DB-02 & & 09/28/02 & CCTV & I & 866 & $\begin{array}{l}\text { CCTV was used to leak check } \\
\text { nozzle } 31 . \text { No leaks were } \\
\text { observed. }\end{array}$ \\
\hline$F$ & DB-02 & MLDB-02 & 01/09/02 & CCTV & I & 807 & $\begin{array}{l}\text { The conductivity probe was } \\
\text { deployed within the setpoint } \\
\text { limits. }\end{array}$ \\
\hline $\mathrm{F}$ & DB-02 & MLDB-03 & 01/09/02 & CCTV & I & 807 & $\begin{array}{l}\text { The conductivity probe was } \\
\text { deployed within the setpoint } \\
\text { limits. }\end{array}$ \\
\hline$F$ & DB-02 & MLDB-03 & 05/13/02 & CCTV & I & $807 A$ & $\begin{array}{l}\text { The conductivity probe was } \\
\text { deployed within the setpoint } \\
\text { limits. }\end{array}$ \\
\hline$F$ & DB-02 & MLDB-03 & $11 / 08 / 02$ & CCTV & / & 841 & $\begin{array}{l}\text { The conductivity probe was } \\
\text { deployed within the setpoint } \\
\text { limits. Mud and corrosion } \\
\text { particles were observed on the } \\
\text { bottom of the MLDB. }\end{array}$ \\
\hline $\mathrm{F}$ & DB-02 & MLDB-04 & 01/04/02 & CCTV & I & 807 & $\begin{array}{l}\text { The conductivity probe was } \\
\text { deployed within the setpoint } \\
\text { limits. The conductivity probe } \\
\text { was touching a mound of solids } \\
\text { located under the standpipe. A } \\
\text { cotton glove and an abandoned } \\
\text { probe were observed in the } \\
\text { bottom of the MLDB. }\end{array}$ \\
\hline $\mathrm{F}$ & DB-02 & MLDB-04 & 08/08/02 & CCTV & I & $809 \mathrm{H}$ & $\begin{array}{l}\text { The conductivity probe was } \\
\text { deployed within the setpoint } \\
\text { limits. }\end{array}$ \\
\hline $\mathrm{F}$ & DB-02 & MLDB-07 & 01/23/02 & CCTV & I & 807 & $\begin{array}{l}\text { The conductivity probe was } \\
\text { deployed within the setpoint } \\
\text { limits. }\end{array}$ \\
\hline $\mathrm{F}$ & DB-02 & MLDB-07 & 03/19/02 & CCTV & I & $807 A$ & $\begin{array}{l}\text { The conductivity probe was } \\
\text { deployed within the setpoint } \\
\text { limits. }\end{array}$ \\
\hline $\mathrm{F}$ & DB-02 & MLDB-07 & 07/25/02 & CCTV & / & 841 & $\begin{array}{l}\text { The conductivity probe was } \\
\text { deployed within the setpoint } \\
\text { limits. }\end{array}$ \\
\hline
\end{tabular}




\begin{tabular}{|c|c|c|c|c|c|c|c|}
\hline \multirow{2}{*}{$\frac{\text { AREA }}{F}$} & \multirow{2}{*}{$\begin{array}{c}\begin{array}{c}\text { TANK OR } \\
\text { ANCILLARY }\end{array} \\
\text { DB-02 }\end{array}$} & \multirow{2}{*}{$\begin{array}{c}\frac{\text { ACCESS OPENING }}{\text { (A OR I) }} \\
\text { Sump }\end{array}$} & \multirow{2}{*}{$\frac{\text { DATE }}{04 / 10 / 02}$} & \multicolumn{3}{|c|}{$\begin{array}{l}\text { INSPECTION METHOD } \\
\text { IDENTIFICATION NUMBER }\end{array}$} & \multirow{2}{*}{$\begin{array}{l}\quad \text { REMARKS } \\
\text { CCTV was used to document } \\
\text { conditions under sump } \\
\text { conductivity probe standpipe. } \\
\text { Liquid and some small solids } \\
\text { were observed on the sump } \\
\text { floor. }\end{array}$} \\
\hline & & & & CCTV & l & $809 E$ & \\
\hline $\mathrm{H}$ & DB-02 & LDB-01 & $01 / 15 / 02$ & CCTV & I & 807 & $\begin{array}{l}\text { The conductivity probe was } \\
\text { deployed within the setpoint } \\
\text { limits. }\end{array}$ \\
\hline $\mathrm{H}$ & DB-02 & LDB-01 & 07/10/02 & CCTV & I & 841 & $\begin{array}{l}\text { The conductivity probe was } \\
\text { deployed within the setpoint } \\
\text { limits. }\end{array}$ \\
\hline $\mathrm{F}$ & DB-03 & & 05/21/02 & CCTV & I & 832 & $\begin{array}{l}\text { CCTV was used to leak check } \\
\text { nozzles } 1,2,3,4,9,10,16,1 A \text {, } \\
2 A, 3 A \text {, and } 4 A \text {. No leaks were } \\
\text { observed. }\end{array}$ \\
\hline $\mathrm{F}$ & DB-04 & & $11 / 13 / 02$ & CCTV & I & 866 & $\begin{array}{l}\text { Inspection revealed that the } \\
\text { shear pin was broken on valve } \\
\text { WTS-V-225. One of the upper } \\
\text { stops was also broken. }\end{array}$ \\
\hline $\mathrm{F}$ & DB-04 & LDB-01 & 02/27/02 & CCTV & I & 807 & $\begin{array}{l}\text { The conductivity probe was } \\
\text { deployed within the setpoint } \\
\text { limits. }\end{array}$ \\
\hline $\mathrm{F}$ & DB-04 & LDB-02 & $10 / 06 / 02$ & CCTV & I & 841 & $\begin{array}{l}\text { The conductivity probe was } \\
\text { deployed within the setpoint } \\
\text { limits. }\end{array}$ \\
\hline $\mathrm{F}$ & DB-04 & LDB-03 & 01/28/02 & CCTV & I & 807 & $\begin{array}{l}\text { The conductivity probe was } \\
\text { deployed within the setpoint } \\
\text { limits. }\end{array}$ \\
\hline $\mathrm{F}$ & DB-04 & LDB-03 & $01 / 31 / 02$ & CCTV & I & 807 & $\begin{array}{l}\text { The conductivity probe was } \\
\text { deployed within the setpoint } \\
\text { limits and was resting on the } \\
\text { bottom of the LDB. Liquid was } \\
\text { observed in the bottom of the } \\
\text { LDB. }\end{array}$ \\
\hline $\mathrm{F}$ & DB-04 & LDB-03 & 01/31/02 & CCTV & I & 807 & $\begin{array}{l}\text { Inspection was performed in } \\
\text { response to a probe alarm. The } \\
\text { probe was raised and reset. The } \\
\text { conductivity probe was deployed } \\
\text { within the setpoint limits. }\end{array}$ \\
\hline $\mathrm{F}$ & DB-04 & LDB-03 & 03/09/02 & CCTV & 1 & $807 A$ & $\begin{array}{l}\text { The conductivity probe was } \\
\text { deployed within the setpoint } \\
\text { limits. }\end{array}$ \\
\hline
\end{tabular}




\begin{tabular}{|c|c|c|c|c|c|c|c|}
\hline \multirow{2}{*}{$\frac{\text { AREA }}{F}$} & \multirow{2}{*}{$\begin{array}{c}\begin{array}{c}\text { TANK OR } \\
\text { ANCILLARY }\end{array} \\
\text { DB-04 }\end{array}$} & \multirow{2}{*}{$\begin{array}{c}\frac{\text { ACCESS OPENING }}{\text { (A OR I) }} \\
\text { LDB-03 }\end{array}$} & \multirow{2}{*}{$\frac{\text { DATE }}{03 / 10 / 02}$} & \multicolumn{3}{|c|}{$\begin{array}{l}\text { INSPECTION METHOD } \\
\text { IDENTIFICATION NUMBER }\end{array}$} & \multirow{2}{*}{$\begin{array}{l}\text { REMARKS } \\
\text { The conductivity probe was } \\
\text { deployed within the setpoint } \\
\text { limits. }\end{array}$} \\
\hline & & & & CCTV & I & $807 \mathrm{~A}$ & \\
\hline $\mathrm{F}$ & DB-04 & LDB-03 & 05/12/02 & CCTV & I & $807 \mathrm{~A}$ & $\begin{array}{l}\text { The conductivity probe was } \\
\text { deployed within the setpoint } \\
\text { limits. }\end{array}$ \\
\hline $\mathrm{F}$ & DB-04 & LDB-03 & $11 / 23 / 02$ & CCTV & I & 841 & $\begin{array}{l}\text { The conductivity probe was } \\
\text { deployed within the setpoint } \\
\text { limits. }\end{array}$ \\
\hline $\mathrm{F}$ & DB-04 & LDB-13 & 03/31/02 & CCTV & I & $807 \mathrm{~A}$ & $\begin{array}{l}\text { The conductivity probe was } \\
\text { deployed within the setpoint } \\
\text { limits. }\end{array}$ \\
\hline $\mathrm{F}$ & DB-04 & LDB-13 & $04 / 11 / 02$ & CCTV & I & $807 \mathrm{~A}$ & $\begin{array}{l}\text { The conductivity probe was } \\
\text { deployed within the setpoint } \\
\text { limits. }\end{array}$ \\
\hline $\mathrm{F}$ & DB-04 & LDB-13 & 05/22/02 & CCTV & I & $807 \mathrm{~A}$ & $\begin{array}{l}\text { The conductivity probe was } \\
\text { deployed within the setpoint } \\
\text { limits. Mud, debris and water } \\
\text { were observed in the bottom of } \\
\text { the LDB. }\end{array}$ \\
\hline $\mathrm{F}$ & DB-04 & LDB-13 & 06/17/02 & CCTV & I & 841 & $\begin{array}{l}\text { The conductivity probe was } \\
\text { deployed within the setpoint } \\
\text { limits. }\end{array}$ \\
\hline $\mathrm{F}$ & DB-04 & LDB-13 & 06/17/02 & CCTV & I & 841 & $\begin{array}{l}\text { The conductivity probe was } \\
\text { deployed within the setpoint } \\
\text { limits. }\end{array}$ \\
\hline $\mathrm{F}$ & DB-04 & LDB-13 & 09/27/02 & CCTV & I & NA & $\begin{array}{l}\text { Inspection revealed liquid in the } \\
\text { LDB. }\end{array}$ \\
\hline $\mathrm{F}$ & DB-04 & LDB-13 & 09/28/02 & CCTV & / & NA & $\begin{array}{l}\text { The conductivity probe was } \\
\text { deployed within the setpoint } \\
\text { limits. }\end{array}$ \\
\hline $\mathrm{F}$ & DB-04 & LDB-13 & 10/30/02 & CCTV & I & 841 & $\begin{array}{l}\text { The conductivity probe was } \\
\text { deployed within the setpoint } \\
\text { limits. }\end{array}$ \\
\hline $\mathrm{F}$ & DB-04 & LDB-13 & $11 / 08 / 02$ & CCTV & / & 841 & $\begin{array}{l}\text { Inspection verified that the } \\
\text { conductivity probe was deployed } \\
\text { within the setpoint limits. The } \\
\text { conductivity probe sheath was } \\
\text { contacting liquid in the LDB. }\end{array}$ \\
\hline $\mathrm{F}$ & DB-04 & LDB-13 & $11 / 09 / 02$ & CCTV & / & 841 & $\begin{array}{l}\text { Inspection revealed liquid and } \\
\text { mud in the LDB. The conductivity } \\
\text { probe was not visible. }\end{array}$ \\
\hline
\end{tabular}




\begin{tabular}{|c|c|c|c|c|c|c|c|}
\hline \multirow{2}{*}{$\frac{\text { AREA }}{F}$} & \multirow{2}{*}{$\begin{array}{c}\text { TANK OR } \\
\text { ANCILLARY } \\
\text { DB-04 }\end{array}$} & \multirow{2}{*}{$\frac{\frac{\text { ACCESS OPENING }}{(\text { A OR I) }}}{\text { LDB }-13}$} & \multirow{2}{*}{$\frac{\text { DATE }}{12 / 26 / 02}$} & \multicolumn{3}{|c|}{$\frac{\text { INSPECTION METHOD }}{\text { IDENTIFICATION NUMBER }}$} & \multirow{2}{*}{$\begin{array}{l}\text { REMARKS } \\
\text { The conductivity probe was } \\
\text { deployed within the setpoint } \\
\text { limits. }\end{array}$} \\
\hline & & & & CCTV & I & 894 & \\
\hline $\mathrm{F}$ & DB-04 & LDB-14 & 08/06/02 & CCTV & / & 841 & $\begin{array}{l}\text { The conductivity probe was } \\
\text { deployed within the setpoint } \\
\text { limits. }\end{array}$ \\
\hline $\mathrm{H}$ & DB-04 & & 04/10/02 & CCTV & / & $809 E$ & $\begin{array}{l}\text { Inspection of the WTS-V-34 } \\
\text { valve port sleeve revealed that } \\
\text { the concrete port had been } \\
\text { enlarged. The original } 4 \text {-inch } \\
\text { sleeve had a larger sleeve core } \\
\text { drilled that encompassed part of } \\
\text { the original sleeve. The valve } \\
\text { cup is below the original sleeve. }\end{array}$ \\
\hline $\mathrm{H}$ & DB-04 & Floor Drain & 01/16/02 & CCTV & I & 809 & $\begin{array}{l}\text { CCTV was used to inspect the } \\
\text { floor drain. An obstruction that } \\
\text { plugged the pipe was observed } \\
\text { approximately } 3 \text { feet down the } \\
\text { drain. }\end{array}$ \\
\hline $\mathrm{H}$ & DB-04 & Floor Drain & 01/17/02 & CCTV & I & $809 A$ & $\begin{array}{l}\text { CCTV was used to inspect the } \\
\text { floor drain. An obstruction that } \\
\text { plugged the pipe was observed } \\
\text { approximately } 3 \text { feet down the } \\
\text { drain. }\end{array}$ \\
\hline $\mathrm{H}$ & DB-04 & LDB-04 & 08/01/02 & CCTV & I & 857 & $\begin{array}{l}\text { The conductivity probe was } \\
\text { deployed within the setpoint } \\
\text { limits. }\end{array}$ \\
\hline $\mathrm{H}$ & DB-06 & LDB-01 & 03/07/02 & CCTV & I & NA & $\begin{array}{l}\text { The conductivity probe was } \\
\text { deployed within the setpoint } \\
\text { limits. }\end{array}$ \\
\hline $\mathrm{H}$ & DB-06 & LDB-02 & 03/07/02 & CCTV & I & NA & $\begin{array}{l}\text { The conductivity probe was } \\
\text { deployed within the setpoint } \\
\text { limits. }\end{array}$ \\
\hline $\mathrm{H}$ & DB-06 & LDB-03 & 03/20/02 & CCTV & I & $807 A$ & $\begin{array}{l}\text { The conductivity probe was } \\
\text { deployed within the setpoint } \\
\text { limits. }\end{array}$ \\
\hline $\mathrm{H}$ & DB-06 & LDB-04 & 03/20/02 & CCTV & I & $807 A$ & $\begin{array}{l}\text { The conductivity probe was } \\
\text { deployed within the setpoint } \\
\text { limits. }\end{array}$ \\
\hline $\mathrm{H}$ & DB-06 & LDB-05 & 03/20/02 & CCTV & I & $807 A$ & $\begin{array}{l}\text { The conductivity probe was } \\
\text { deployed within the setpoint } \\
\text { limits. }\end{array}$ \\
\hline $\mathrm{H}$ & DB-06 & LDB-05 & 06/04/02 & CCTV & I & $807 A$ & $\begin{array}{l}\text { The conductivity probe was } \\
\text { deployed within the setpoint } \\
\text { limits. }\end{array}$ \\
\hline
\end{tabular}




\begin{tabular}{|c|c|c|c|c|c|c|c|}
\hline \multirow{2}{*}{$\frac{\text { AREA }}{\mathrm{H}}$} & \multirow{2}{*}{$\begin{array}{c}\text { TANK OR } \\
\text { ANCILLARY } \\
\text { DB-06 }\end{array}$} & \multirow{2}{*}{$\frac{\frac{\text { ACCESS OPENING }}{\text { (A OR I) }}}{\text { LDB-05 }}$} & \multirow{2}{*}{$\frac{\text { DATE }}{07 / 07 / 02}$} & \multicolumn{3}{|c|}{$\begin{array}{l}\text { INSPECTION METHOD } \\
\text { IDENTIFICATION NUMBER }\end{array}$} & \multirow{2}{*}{$\begin{array}{l}\text { REMARKS } \\
\text { The conductivity probe was } \\
\text { deployed within the setpoint } \\
\text { limits. }\end{array}$} \\
\hline & & & & CCTV & I & 841 & \\
\hline $\mathrm{H}$ & DB-06 & LDB-05 & 08/18/02 & CCTV & I & 841 & $\begin{array}{l}\text { The conductivity probe was } \\
\text { deployed within the setpoint } \\
\text { limits. }\end{array}$ \\
\hline $\mathrm{H}$ & DB-07 & & 04/21/02 & CCTV & I & $809 E$ & $\begin{array}{l}\text { Inspection was made to } \\
\text { document the flush water piping } \\
\text { configuration between the valve } \\
\text { box and the diversion box. }\end{array}$ \\
\hline $\mathrm{H}$ & DB-07 & & $10 / 03 / 02$ & CCTV & I & 873 & $\begin{array}{l}\text { CCTV was used to document the } \\
\text { conditions of FW-117 valve } \\
\text { body. No unusual conditions } \\
\text { were observed. }\end{array}$ \\
\hline $\mathrm{H}$ & DB-07 & LDB-01 & 03/04/02 & CCTV & I & $807 A$ & $\begin{array}{l}\text { The conductivity probe was } \\
\text { deployed within the setpoint } \\
\text { limits. }\end{array}$ \\
\hline $\mathrm{H}$ & DB-07 & LDB-01 & 08/27/02 & CCTV & l & 841 & $\begin{array}{l}\text { The conductivity probe was } \\
\text { deployed within the setpoint } \\
\text { limits. }\end{array}$ \\
\hline $\mathrm{H}$ & DB-07 & LDB-02 & 03/06/02 & CCTV & l & $807 A$ & $\begin{array}{l}\text { The conductivity probe was } \\
\text { deployed within the setpoint } \\
\text { limits. }\end{array}$ \\
\hline $\mathrm{H}$ & DB-07 & LDB-02 & $10 / 29 / 02$ & CCTV & l & 841 & $\begin{array}{l}\text { The conductivity probe was } \\
\text { deployed within the setpoint } \\
\text { limits. }\end{array}$ \\
\hline $\mathrm{H}$ & DB-07 & LDB-03 & $01 / 24 / 02$ & CCTV & I & 807 & $\begin{array}{l}\text { The conductivity probe was } \\
\text { deployed within the setpoint } \\
\text { limits. }\end{array}$ \\
\hline $\mathrm{H}$ & DB-07 & LDB-03 & 07/23/02 & CCTV & l & 841 & $\begin{array}{l}\text { The conductivity probe was } \\
\text { deployed within the setpoint } \\
\text { limits. }\end{array}$ \\
\hline $\mathrm{H}$ & DB-07 & LDB-04 & 05/02/02 & CCTV & l & $807 A$ & $\begin{array}{l}\text { The conductivity probe was } \\
\text { deployed within the setpoint } \\
\text { limits. }\end{array}$ \\
\hline $\mathrm{H}$ & DB-07 & LDB-04 & $10 / 29 / 02$ & CCTV & I & 841 & $\begin{array}{l}\text { The conductivity probe was } \\
\text { deployed within the setpoint } \\
\text { limits. }\end{array}$ \\
\hline $\mathrm{H}$ & DB-07 & LDB-05 & $01 / 24 / 02$ & CCTV & l & 807 & $\begin{array}{l}\text { The conductivity probe was } \\
\text { deployed within the setpoint } \\
\text { limits. }\end{array}$ \\
\hline
\end{tabular}




\begin{tabular}{|c|c|c|c|c|c|c|c|}
\hline \multirow{2}{*}{$\frac{\text { AREA }}{\mathrm{H}}$} & \multirow{2}{*}{$\begin{array}{c}\begin{array}{c}\text { TANK OR } \\
\text { ANCILLARY }\end{array} \\
\text { DB-07 }\end{array}$} & \multirow{2}{*}{$\begin{array}{c}\frac{\text { ACCESS OPENING }}{\text { (A OR I) }} \\
\text { LDB-06 }\end{array}$} & \multirow{2}{*}{$\frac{\text { DATE }}{05 / 21 / 02}$} & \multicolumn{3}{|c|}{$\begin{array}{l}\text { INSPECTION METHOD } \\
\text { IDENTIFICATION NUMBER }\end{array}$} & \multirow{2}{*}{$\begin{array}{l}\text { REMARKS } \\
\text { The conductivity probe was } \\
\text { deployed within the setpoint } \\
\text { limits. }\end{array}$} \\
\hline & & & & CCTV & I & $807 \mathrm{~A}$ & \\
\hline $\mathrm{H}$ & DB-07 & LDB-06 & $11 / 13 / 02$ & CCTV & I & 841 & $\begin{array}{l}\text { The conductivity probe was } \\
\text { deployed within the setpoint } \\
\text { limits. }\end{array}$ \\
\hline $\mathrm{H}$ & DB-07 & LDB-07 & 05/10/02 & CCTV & I & $807 \mathrm{~A}$ & $\begin{array}{l}\text { The conductivity probe was } \\
\text { deployed within the setpoint } \\
\text { limits. Debris and liquid were } \\
\text { observed on the bottom of the } \\
\text { LDB. }\end{array}$ \\
\hline $\mathrm{H}$ & DB-07 & LDB-07 & $05 / 21 / 02$ & CCTV & I & $807 \mathrm{~A}$ & $\begin{array}{l}\text { The conductivity probe was } \\
\text { deployed within the setpoint } \\
\text { limits. }\end{array}$ \\
\hline $\mathrm{H}$ & DB-07 & LDB-07 & 05/22/02 & CCTV & I & $807 \mathrm{~A}$ & $\begin{array}{l}\text { The conductivity probe was } \\
\text { deployed within the setpoint } \\
\text { limits. }\end{array}$ \\
\hline $\mathrm{H}$ & DB-07 & LDB-07 & $12 / 08 / 02$ & CCTV & I & 894 & $\begin{array}{l}\text { The conductivity probe was } \\
\text { deployed within the setpoint } \\
\text { limits. }\end{array}$ \\
\hline $\mathrm{H}$ & DB-07 & LDB-08 & 03/06/02 & CCTV & I & $807 \mathrm{~A}$ & $\begin{array}{l}\text { The conductivity probe was } \\
\text { deployed within the setpoint } \\
\text { limits. }\end{array}$ \\
\hline $\mathrm{H}$ & DB-07 & LDB-08 & 08/27/02 & CCTV & I & 841 & $\begin{array}{l}\text { The conductivity probe was } \\
\text { deployed within the setpoint } \\
\text { limits. }\end{array}$ \\
\hline $\mathrm{H}$ & DB-08 & & 01/16/02 & CCTV & I & $809 \mathrm{~A}$ & $\begin{array}{l}\text { CCTV was used to verify that the } \\
\text { new WTS-V-200 valve body } \\
\text { would cycle properly. }\end{array}$ \\
\hline $\mathrm{H}$ & $\begin{array}{l}\text { East Hill } \\
\text { Drain Cell }\end{array}$ & & 01/10/02 & CCTV & I & 807 & $\begin{array}{l}\text { CCTV was used to document } \\
\text { conditions of the drain cell sump. } \\
\text { A small amount of liquid was } \\
\text { observed. The conductivity } \\
\text { probe could not be properly } \\
\text { positioned due to incorrect } \\
\text { markings on the probe. }\end{array}$ \\
\hline $\mathrm{H}$ & $\begin{array}{l}\text { East Hill } \\
\text { Drain Cell }\end{array}$ & & 01/14/02 & CCTV & I & NA & $\begin{array}{l}\text { The conductivity probe could not } \\
\text { be deployed due to liquid in the } \\
\text { cell. }\end{array}$ \\
\hline $\mathrm{H}$ & $\begin{array}{l}\text { East Hill } \\
\text { Drain Cell }\end{array}$ & & 01/16/02 & CCTV & I & 807 & $\begin{array}{l}\text { The conductivity probe was } \\
\text { deployed within the setpoint } \\
\text { limits. }\end{array}$ \\
\hline
\end{tabular}




\begin{tabular}{|c|c|c|c|c|c|c|c|}
\hline \multirow{2}{*}{$\frac{\text { AREA }}{\mathrm{H}}$} & \multirow{2}{*}{$\begin{array}{c}\text { TANK OR } \\
\text { ANCILLARY } \\
\text { East Hill } \\
\text { Drain Cell }\end{array}$} & $\frac{\text { ACCESS OPENING }}{(\text { A OR I) }}$ & \multirow{2}{*}{$\frac{\text { DATE }}{07 / 05 / 02}$} & \multicolumn{3}{|c|}{$\begin{array}{l}\text { INSPECTION METHOD } \\
\text { IDENTIFICATION NUMBER }\end{array}$} & \multirow{2}{*}{$\begin{array}{l}\text { REMARKS } \\
\text { Inspection revealed standing } \\
\text { liquid in the bottom of the drain } \\
\text { cell. No inleakage into the cell } \\
\text { was observed. }\end{array}$} \\
\hline & & & & CCTV & / & NA & \\
\hline $\mathrm{H}$ & $\begin{array}{l}\text { East Hill } \\
\text { Drain Cell }\end{array}$ & & 07/28/02 & CCTV & I & $809 \mathrm{H}$ & $\begin{array}{l}\text { Inspection revealed that the } \\
\text { conductivity probe extended } \\
\text { below the standpipe. } \\
\text { Abandoned cables and hoses } \\
\text { were observed in the sump. }\end{array}$ \\
\hline $\mathrm{H}$ & $\begin{array}{l}\text { East Hill } \\
\text { Drain Cell }\end{array}$ & $\begin{array}{r}\text { Underliner } \\
\text { Sump }\end{array}$ & $01 / 01 / 02$ & CCTV & I & 807 & $\begin{array}{l}\text { CCTV was used to document } \\
\text { conditions of underliner sump } \\
\text { conductivity probe. The probe } \\
\text { was submerged and resting on } \\
\text { debris. }\end{array}$ \\
\hline $\mathrm{H}$ & $\begin{array}{l}\text { East Hill } \\
\text { Drain Cell }\end{array}$ & $\begin{array}{r}\text { Underliner } \\
\text { Sump }\end{array}$ & 01/02/02 & CCTV & I & 807 & $\begin{array}{l}\text { CCTV was used to document } \\
\text { conditions of underliner sump } \\
\text { conductivity probe. Liquid was } \\
\text { observed in the standpipe. }\end{array}$ \\
\hline $\mathrm{H}$ & $\begin{array}{l}\text { East Pump } \\
\text { House }\end{array}$ & & $12 / 05 / 02$ & CCTV & I & 8091 & $\begin{array}{l}\text { Inspection of the heat exchanger } \\
\text { revealed no significant deposits } \\
\text { on the tube bundle or unusual } \\
\text { conditions. Deposits were } \\
\text { observed on the tubes beneath } \\
\text { the access points and on the } \\
\text { heat exchanger interior walls. }\end{array}$ \\
\hline $\mathrm{F}$ & EVAP-01 & & 02/13/02 & CCTV & I & 812 & $\begin{array}{l}\text { Inspection revealed } \\
\text { approximately } 2 \text { to } 3 \text { inches of } \\
\text { liquid in the cell with a few } \\
\text { extraneous items and trash on } \\
\text { the cell floor. }\end{array}$ \\
\hline$F$ & EVAP-01 & C (I) & 02/13/02 & CCTV & I & 812 & $\begin{array}{l}\text { Inspection of the vessel interior } \\
\text { revealed a very light scale on all } \\
\text { of the exposed surfaces. No } \\
\text { accumulation of solids or } \\
\text { deposits were observed on the } \\
\text { warming coil, tube bundle, vessel } \\
\text { walls, or the exposed surfaces. } \\
\text { Approximately } 5 \text { feet of clear } \\
\text { liquid remained in the vessel and } \\
\text { less than } 1 \text { inch of fine loose } \\
\text { solids were present in the bottom } \\
\text { of the vessel. No unusual } \\
\text { conditions were observed, and } \\
\text { the condition of the vessel was } \\
\text { consistent with normal operation. }\end{array}$ \\
\hline $\mathrm{F}$ & EVAP-01 & C (I) & 02/28/02 & CCTV & I & 812 & $\begin{array}{l}\text { Inspection revealed less than } 1 \\
\text { inch of fine loose solids at the } \\
\text { base of the conical region. }\end{array}$ \\
\hline
\end{tabular}




\begin{tabular}{|c|c|c|c|c|c|c|c|}
\hline \multirow{2}{*}{$\frac{\text { AREA }}{F}$} & \multirow{2}{*}{$\begin{array}{c}\begin{array}{c}\text { TANK OR } \\
\text { ANCILLARY }\end{array} \\
\text { EVAP-16 }\end{array}$} & \multirow{2}{*}{$\begin{array}{c}\frac{\text { ACCESS OPENING }}{\text { (A OR I) }} \\
\text { SE }\end{array}$} & \multirow{2}{*}{$\frac{\text { DATE }}{07 / 17 / 02}$} & \multicolumn{3}{|c|}{$\begin{array}{l}\text { INSPECTION METHOD } \\
\text { IDENTIFICATION NUMBER }\end{array}$} & \multirow{2}{*}{$\begin{array}{l}\quad \text { REMARKS } \\
\text { CCTV was used to leak check } \\
\text { jumper } 4(2 \mathrm{~F} \text { Evap)5. No leaks } \\
\text { were observed. }\end{array}$} \\
\hline & & & & CCTV & I & 851 & \\
\hline $\mathrm{F}$ & EVAP-16 & SE & 07/18/02 & CCTV & I & 851 & $\begin{array}{l}\text { CCTV was used to leak check } \\
\text { jumper } 4(2 \mathrm{~F} \text { Evap }) 5 . \text { No leaks } \\
\text { were observed. }\end{array}$ \\
\hline$F$ & EVAP-16 & $\begin{array}{r}\text { Separator } \\
\text { Pot }\end{array}$ & $12 / 30 / 02$ & CCTV & I & 904 & $\begin{array}{l}\text { Inspection of the outlet side of } \\
\text { the separator pot revealed no } \\
\text { significant deposits. } \\
\text { Approximately } 24 \text { feet of the } \\
\text { vertical section of the GDL to } \\
\text { Tank } 46 \text { was inspected. }\end{array}$ \\
\hline $\mathrm{F}$ & EVAP-16 & SW & 09/23/02 & CCTV & I & 851 & $\begin{array}{l}\text { Inspection documented the } \\
\text { conditions of the evaporator cell, } \\
\text { pot exterior, and service piping. } \\
\text { No unusual conditions were } \\
\text { observed. }\end{array}$ \\
\hline $\mathrm{H}$ & EVAP-16 & & 07/03/02 & CCTV & I & 848 & $\begin{array}{l}\text { CCTV was used to document } \\
\text { the removal of the E-3 guide } \\
\text { tube and the condition of the } \\
\text { nozzle face. The face of the } \\
\text { nozzle appeared to be normal; } \\
\text { however, some gasket material } \\
\text { remained on the nozzle face. No } \\
\text { other unusual conditions were } \\
\text { observed. A dummy nozzle was } \\
\text { installed. }\end{array}$ \\
\hline $\mathrm{H}$ & EVAP-16 & & $12 / 07 / 02$ & CCTV & l & 848 & $\begin{array}{l}\text { CCTV documented conditions in } \\
\text { the cell. No unusual conditions } \\
\text { were observed. }\end{array}$ \\
\hline $\mathrm{H}$ & EVAP-16 & $\begin{array}{r}\text { Condenser } \\
\text { Cell }\end{array}$ & $01 / 14 / 02$ & CCTV & I & $781 \mathrm{D}$ & $\begin{array}{l}\text { CCTV was used to determine if } \\
\text { the leak had increased at the } \\
\text { condenser flange after an } \\
\text { increase in the sump was } \\
\text { observed. The leak had not } \\
\text { increased since last inspected, } \\
\text { and the sump level increase was } \\
\text { attributed to rainwater inleakage. }\end{array}$ \\
\hline $\mathrm{H}$ & EVAP-16 & $\begin{array}{r}\text { Condenser } \\
\text { Cell }\end{array}$ & $01 / 25 / 02$ & CCTV & I & 810 & $\begin{array}{l}\text { Inspection verified that the } \\
\text { leakage from the upper flange on } \\
\text { the condenser had not increased } \\
\text { since last inspected. }\end{array}$ \\
\hline $\mathrm{H}$ & EVAP-16 & $\begin{array}{r}\text { Condenser } \\
\text { Cell }\end{array}$ & 02/06/02 & CCTV & I & 810 & $\begin{array}{l}\text { Inspection verified that the } \\
\text { leakage from the upper flange on } \\
\text { the condenser had not increased } \\
\text { since last inspected. }\end{array}$ \\
\hline
\end{tabular}




\begin{tabular}{|c|c|c|c|c|c|c|c|}
\hline \multirow{2}{*}{$\frac{\text { AREA }}{\mathrm{H}}$} & \multirow{2}{*}{$\begin{array}{c}\text { TANK OR } \\
\text { ANCILLARY } \\
\text { EVAP-16 }\end{array}$} & \multirow{2}{*}{$\begin{array}{c}\frac{\text { ACCESS OPENING }}{(\text { A OR I) }} \\
\begin{array}{c}\text { Condenser } \\
\text { Cell }\end{array}\end{array}$} & \multirow{2}{*}{$\frac{\text { DATE }}{02 / 19 / 02}$} & \multicolumn{3}{|c|}{$\begin{array}{l}\text { INSPECTION METHOD } \\
\text { IDENTIFICATION NUMBER }\end{array}$} & \multirow{2}{*}{$\begin{array}{l}\text { REMARKS } \\
\text { Inspection verified that the } \\
\text { leakage from the upper flange on } \\
\text { the condenser had not increased } \\
\text { since last inspected. }\end{array}$} \\
\hline & & & & CCTV & I & 810 & \\
\hline $\mathrm{H}$ & EVAP-16 & $\begin{array}{r}\text { Condenser } \\
\text { Cell }\end{array}$ & 03/02/02 & CCTV & I & 810 & $\begin{array}{l}\text { Inspection verified that the } \\
\text { leakage from the upper flange on } \\
\text { the condenser had not increased } \\
\text { since last inspected. }\end{array}$ \\
\hline $\mathrm{H}$ & EVAP-16 & $\begin{array}{r}\text { Condenser } \\
\text { Cell }\end{array}$ & 03/09/02 & CCTV & I & 810 & $\begin{array}{l}\text { Inspection verified that the } \\
\text { leakage from the upper flange on } \\
\text { the condenser had not increased } \\
\text { since last inspected. }\end{array}$ \\
\hline $\mathrm{H}$ & EVAP-16 & $\begin{array}{r}\text { Condenser } \\
\text { Cell }\end{array}$ & 03/26/02 & CCTV & I & 810 & $\begin{array}{l}\text { Inspection verified that the } \\
\text { leakage from the upper flange on } \\
\text { the condenser had not increased } \\
\text { since last inspected. }\end{array}$ \\
\hline $\mathrm{H}$ & EVAP-16 & $\begin{array}{r}\text { Condenser } \\
\text { Cell }\end{array}$ & 03/30/02 & CCTV & I & 810 & $\begin{array}{l}\text { Inspection verified that the } \\
\text { leakage from the upper flange on } \\
\text { the condenser had not increased } \\
\text { since last inspected. }\end{array}$ \\
\hline $\mathrm{H}$ & EVAP-16 & $\begin{array}{r}\text { Condenser } \\
\text { Cell }\end{array}$ & 04/10/02 & CCTV & l & 810 & $\begin{array}{l}\text { Inspection verified that the } \\
\text { leakage from the upper flange on } \\
\text { the condenser had not increased } \\
\text { since last inspected. }\end{array}$ \\
\hline $\mathrm{H}$ & EVAP-16 & $\begin{array}{r}\text { Condenser } \\
\text { Cell }\end{array}$ & 04/18/02 & CCTV & I & 810 & $\begin{array}{l}\text { Inspection verified that the } \\
\text { leakage from the upper flange on } \\
\text { the condenser had not increased } \\
\text { since last inspected. }\end{array}$ \\
\hline $\mathrm{H}$ & EVAP-16 & $\begin{array}{r}\text { Condenser } \\
\text { Cell }\end{array}$ & $04 / 25 / 02$ & CCTV & I & 810 & $\begin{array}{l}\text { Inspection verified that the } \\
\text { leakage from the upper flange on } \\
\text { the condenser had not increased } \\
\text { since last inspected. }\end{array}$ \\
\hline $\mathrm{H}$ & EVAP-16 & $\begin{array}{r}\text { Condenser } \\
\text { Cell }\end{array}$ & $04 / 25 / 02$ & CCTV & I & 810 & $\begin{array}{l}\text { Inspection verified that the } \\
\text { leakage from the upper flange on } \\
\text { the condenser had not increased } \\
\text { since last inspected. }\end{array}$ \\
\hline $\mathrm{H}$ & EVAP-16 & $\begin{array}{r}\text { Condenser } \\
\text { Cell }\end{array}$ & 05/01/02 & CCTV & I & 810 & $\begin{array}{l}\text { Inspection verified that the } \\
\text { leakage from the upper flange on } \\
\text { the condenser had not increased } \\
\text { since last inspected. }\end{array}$ \\
\hline $\mathrm{H}$ & EVAP-16 & $\begin{array}{r}\text { Condenser } \\
\text { Cell }\end{array}$ & 07/28/02 & CCTV & I & 848 & $\begin{array}{l}\text { Inspection verified that the } \\
\text { leakage from the upper flange on } \\
\text { the condenser had not increased } \\
\text { since last inspected. }\end{array}$ \\
\hline
\end{tabular}




\begin{tabular}{|c|c|c|c|c|c|c|c|}
\hline \multirow{2}{*}{$\frac{\text { AREA }}{\mathrm{H}}$} & \multirow{2}{*}{$\begin{array}{c}\text { TANK OR } \\
\text { ANCILLARY } \\
\text { EVAP-16 }\end{array}$} & $\frac{\text { ACCESS OPENING }}{(\text { A OR I) }}$ & \multirow{2}{*}{$\frac{\text { DATE }}{07 / 30 / 02}$} & \multicolumn{3}{|c|}{$\frac{\text { INSPECTION METHOD }}{\text { IDENTIFICATION NUMBER }}$} & \multirow{2}{*}{$\begin{array}{l}\text { REMARKS } \\
\text { pection verified that the } \\
\text { kage from the upper flange on } \\
\text { condenser had not increased } \\
\text { ce last inspected. }\end{array}$} \\
\hline & & $\begin{array}{r}\text { Condenser } \\
\text { Cell }\end{array}$ & & CCTV & I & 848 & \\
\hline $\mathrm{H}$ & EVAP-16 & $\begin{array}{r}\text { Condenser } \\
\text { Cell }\end{array}$ & $11 / 27 / 02$ & CCTV & I & 848 & $\begin{array}{l}\text { Inspection was performed to } \\
\text { investigate increase in the } \\
\text { evaporator sump level. No } \\
\text { unusual conditions were } \\
\text { observed. }\end{array}$ \\
\hline $\mathrm{H}$ & EVAP-16 & Open Cell & 03/18/02 & CCTV & I & 819 & $\begin{array}{l}\text { Inspection documented the cell } \\
\text { walls, jumper connections and } \\
\text { some of the cell floor. No } \\
\text { unusual conditions were } \\
\text { observed. }\end{array}$ \\
\hline $\mathrm{H}$ & EVAP-16 & SW & 03/25/02 & CCTV & I & 810 & $\begin{array}{l}\text { CCTV was used to leak check } \\
\text { the E-3 Hanford connector. No } \\
\text { leaks were observed. }\end{array}$ \\
\hline $\mathrm{H}$ & EVAP-16 & SW & 06/28/02 & CCTV & I & 810 & $\begin{array}{l}\text { CCTV was used to leak check } \\
\text { the E-3 Hanford connector. No } \\
\text { leaks were observed. }\end{array}$ \\
\hline $\mathrm{H}$ & EVAP-16 & SW & 07/04/02 & CCTV & I & 848 & $\begin{array}{l}\text { CCTV was used to leak check } \\
\text { the E-3 Hanford connector. No } \\
\text { leaks were observed. }\end{array}$ \\
\hline $\mathrm{H}$ & EVAP-16 & SW & 09/16/02 & CCTV & I & 848 & $\begin{array}{l}\text { Inspection documented the } \\
\text { condition of the evaporator pot } \\
\text { exterior and cell. No unusual } \\
\text { conditions were observed. Small } \\
\text { pieces of plastic were observed } \\
\text { in the sump. }\end{array}$ \\
\hline$F$ & EVAP-16 & D (I) & 07/16/02 & CCTV & I & 851 & $\begin{array}{l}\text { CCTV was used to inspect the } \\
\text { interior of the evaporator pot. No } \\
\text { deposits were observed on the } \\
\text { pot wall, tube bundle, warming } \\
\text { coil, demister spray ring or } \\
\text { service piping. A very thin scale } \\
\text { was observed on the tube } \\
\text { bundle. A small amount of loose } \\
\text { solids had settled on the piping } \\
\text { beneath the operating level. } \\
\text { Visibility through the remaining } \\
\text { liquid, approximately } 12 \text { inches, } \\
\text { allowed inspection to within } 6 \\
\text { inches of the bottom of the cone. } \\
\text { Observations in this inspection } \\
\text { were consistent with normal } \\
\text { evaporator operation. }\end{array}$ \\
\hline
\end{tabular}




\begin{tabular}{|c|c|c|c|c|c|c|c|}
\hline \multirow{2}{*}{$\frac{\text { AREA }}{F}$} & \multirow{2}{*}{$\begin{array}{c}\text { TANK OR } \\
\text { ANCILLARY } \\
\text { EVAP-16 }\end{array}$} & $\frac{\text { ACCESS OPENING }}{(\text { A OR I) }}$ & \multirow{2}{*}{$\frac{\text { DATE }}{12 / 31 / 02}$} & \multicolumn{3}{|c|}{$\frac{\text { INSPECTION METHOD }}{\text { IDENTIFICATION NUMBER }}$} & \multirow[b]{2}{*}{$\begin{array}{l}\text { REMARKS } \\
\text { Inspection revealed a thin scale } \\
\text { on the pot walls and service } \\
\text { piping. Deposits were observed } \\
\text { on the tube bundle; however, } \\
\text { these deposits were mostly likely } \\
\text { due to the waste left in the pot } \\
\text { while the GDL was plugged. The } \\
\text { deposits observed were not } \\
\text { similar in appearance to the } \\
\text { buildup observed during the } 2 \mathrm{H} \\
\text { evaporator pblockages. }\end{array}$} \\
\hline & & E-04 (I) & & CCTV & I & 904 & \\
\hline
\end{tabular}

CCTV was used to inspect the interior of the evaporator pot. Observations were consistent with normal operation. No deposits were observed. A thin scaling of approximately $1 / 32$ inches was observed on the pot walls, tube bundle, warming coil, service piping and spray ring. Approximately 3 to 4 feet of liquid remained in the pot which prevented observations of the bottom of the cone.

Inspection revealed no significant changes since last inspected on $3 / 10 / 02$. A very thin scale was observed on the tube bundle, service piping, warming coil and vessel wall. Some loose solids had settled on top of the warming coil during liquid removal. Approximately 7.5 inches of liquid and less than 1 inch of solids remained in the pot. Observations in this inspection were consistent with normal evaporator operation.

Inspection of the pot interior show no large areas of deposits since last inspected. Deposits observed on the vessel wall near the feed jumper are less than 0.25 inches. A thin scale was observed on the vessel wall, tube bundle, warming coil and service piping. Small amounts of loose solids had settled on the piping beneath the operating level. Observations from this inspection were consistent with normal operations. 


\begin{tabular}{|c|c|c|c|c|c|c|c|}
\hline \multirow{2}{*}{$\frac{\text { AREA }}{\mathrm{H}}$} & \multirow{2}{*}{$\begin{array}{c}\begin{array}{c}\text { TANK OR } \\
\text { ANCILLARY }\end{array} \\
\text { EVAP-25 }\end{array}$} & \multirow[t]{2}{*}{$\frac{\text { ACCESS OPENING }}{\text { (A OR I) }}$} & \multirow{2}{*}{$\frac{\text { DATE }}{06 / 05 / 02}$} & \multicolumn{3}{|c|}{$\begin{array}{l}\text { INSPECTION METHOD } \\
\text { IDENTIFICATION NUMBER }\end{array}$} & \multirow{2}{*}{$\begin{array}{l}\quad \text { REMARKS } \\
\text { Inspection verified that the } \\
\text { condensate cell vent line was } \\
\text { unobstructed. }\end{array}$} \\
\hline & & & & CCTV & l & 837 & \\
\hline $\mathrm{H}$ & EVAP-25 & & 06/05/02 & CCTV & I & 837 & $\begin{array}{l}\text { Inspection revealed that the lift } \\
\text { jumper brace was contacting the } \\
\text { P21 nozzle shelf. }\end{array}$ \\
\hline $\mathrm{H}$ & EVAP-25 & & 07/19/02 & CCTV & I & 852 & $\begin{array}{l}\text { Inspection verified that the } \\
\text { condensate cell vent line was } \\
\text { unobstructed. }\end{array}$ \\
\hline $\mathrm{H}$ & EVAP-25 & & 07/25/02 & CCTV & l & 853 & $\begin{array}{l}\text { CCTV was used to leak check } \\
\text { jumpers Y555-100-140, } \\
\text { Y555-100-124-3, and } \\
\text { Y555-100-121. No leaks were } \\
\text { observed. }\end{array}$ \\
\hline $\mathrm{H}$ & EVAP-25 & & 08/13/02 & CCTV & l & 853 & $\begin{array}{l}\text { CCTV was used to document the } \\
\text { configuration of the Resistance } \\
\text { Thermo Device jumper. The } \\
\text { ceramic plate on the electrical } \\
\text { nozzle face was cracked on the } \\
\text { separator pot side with no } \\
\text { unusual conditions observed on } \\
\text { the E-01 electrical face. The } \\
\text { Y555-100-133 electrical jumper } \\
\text { appeared to be normal. }\end{array}$ \\
\hline $\mathrm{H}$ & EVAP-25 & & 08/14/02 & CCTV & I & 853 & $\begin{array}{l}\text { CCTV was used to document the } \\
\text { configuration of the electrical } \\
\text { pins on the upper and lower } \\
\text { plates of the Resistance Thermo } \\
\text { Device jumper. } \\
\text { The upper and lower plates did } \\
\text { not match. }\end{array}$ \\
\hline $\mathrm{H}$ & EVAP-25 & & 08/28/02 & CCTV & I & 853 & $\begin{array}{l}\text { Inspection verified that the } \\
\text { condenser cell vent line was } \\
\text { unobstructed. }\end{array}$ \\
\hline $\mathrm{H}$ & EVAP-25 & & 09/12/02 & CCTV & I & NA & $\begin{array}{l}\text { CCTV documented thermal } \\
\text { expansion of the evaporator } \\
\text { vessel as it reached operating } \\
\text { temperature. }\end{array}$ \\
\hline $\mathrm{H}$ & EVAP-25 & $\mathrm{NE}$ & 03/06/02 & CCTV & l & $811 \mathrm{D}$ & $\begin{array}{l}\text { Inspection revealed salt deposits } \\
\text { at the U2 nozzle indicating that } \\
\text { the connector head is leaking. }\end{array}$ \\
\hline $\mathrm{H}$ & EVAP-25 & $\mathrm{NE}$ & 03/09/02 & CCTV & l & $811 \mathrm{D}$ & $\begin{array}{l}\text { Inspection documented a leak at } \\
\text { the U2 connector during a lift out } \\
\text { of the pot and backflush. }\end{array}$ \\
\hline
\end{tabular}




\begin{tabular}{|c|c|c|c|c|c|c|c|}
\hline \multirow{2}{*}{$\frac{\text { AREA }}{\mathrm{H}}$} & \multirow{2}{*}{$\begin{array}{c}\text { TANK OR } \\
\text { ANCILLARY } \\
\text { EVAP-25 }\end{array}$} & \multirow{2}{*}{$\frac{\frac{\text { ACCESS OPENING }}{\text { (A OR I) }}}{\text { NE }}$} & \multirow{2}{*}{$\frac{\text { DATE }}{03 / 22 / 02}$} & \multicolumn{3}{|c|}{$\begin{array}{l}\text { INSPECTION METHOD } \\
\text { IDENTIFICATION NUMBER }\end{array}$} & \multirow{2}{*}{$\begin{array}{l}\frac{\text { REMARKS }}{\text { CCTV was used to leak check }} \\
\text { jumper Y555-100-121\&124. No } \\
\text { leaks were observed. }\end{array}$} \\
\hline & & & & CCTV & I & $811 \mathrm{H}$ & \\
\hline $\mathrm{H}$ & EVAP-25 & NE & 03/23/02 & CCTV & I & $811 \mathrm{H}$ & $\begin{array}{l}\text { CCTV was used to leak check } \\
\text { valve } 1055 . \text { No leaks were } \\
\text { observed. }\end{array}$ \\
\hline $\mathrm{H}$ & EVAP-25 & NE & 03/26/02 & CCTV & I & $811 \mathrm{H}$ & $\begin{array}{l}\text { CCTV was used to leak check } \\
\text { valve } 1055 \text { and jumper } \\
\text { Y-555-100-121\&124. No leaks } \\
\text { were observed. }\end{array}$ \\
\hline $\mathrm{H}$ & EVAP-25 & NE & 03/30/02 & CCTV & I & $811 G$ & $\begin{array}{l}\text { Inspection revealed salt deposits } \\
\text { on the U2 nozzle plate. }\end{array}$ \\
\hline $\mathrm{H}$ & EVAP-25 & NE & 03/30/02 & CCTV & I & $811 G$ & $\begin{array}{l}\text { Inspection revealed liquid and } \\
\text { salt deposits on the cell floor. }\end{array}$ \\
\hline $\mathrm{H}$ & EVAP-25 & NE & 04/03/02 & CCTV & I & 8111 & $\begin{array}{l}\text { CCTV was used to determine if } \\
\text { the connector head on lift } \\
\text { jumper Y555-100-121 and the } \\
\text { U2 nozzle were properly aligned. } \\
\text { The jumper appeared to be } \\
\text { properly aligned. }\end{array}$ \\
\hline $\mathrm{H}$ & EVAP-25 & NE & 04/15/02 & CCTV & I & $811 \mathrm{~J}$ & $\begin{array}{l}\text { CCTV was used to check for } \\
\text { leakage at the U2 and P5 } \\
\text { nozzles. No leaks were } \\
\text { observed. }\end{array}$ \\
\hline $\mathrm{H}$ & EVAP-25 & NE & 04/19/02 & CCTV & I & $811 \mathrm{~J}$ & $\begin{array}{l}\text { CCTV was used to check for } \\
\text { leakage at the U2 and P5 } \\
\text { nozzles. No leaks were } \\
\text { observed. }\end{array}$ \\
\hline $\mathrm{H}$ & EVAP-25 & NE & 04/20/02 & CCTV & I & $811 \mathrm{~J}$ & $\begin{array}{l}\text { CCTV was used to check for } \\
\text { leakage at the U2 and P5 } \\
\text { nozzles. No leaks were } \\
\text { observed. }\end{array}$ \\
\hline $\mathrm{H}$ & EVAP-25 & NE & 04/21/02 & CCTV & I & $811 \mathrm{~J}$ & $\begin{array}{l}\text { CCTV was used to check for } \\
\text { leakage at the U2 and P5 } \\
\text { nozzles. No leaks were } \\
\text { observed. }\end{array}$ \\
\hline $\mathrm{H}$ & EVAP-25 & NE & 04/22/02 & CCTV & I & $811 \mathrm{~J}$ & $\begin{array}{l}\text { CCTV was used to check for } \\
\text { leakage at the U2 and P5 } \\
\text { nozzles. No leaks were } \\
\text { observed. }\end{array}$ \\
\hline $\mathrm{H}$ & EVAP-25 & NE & 04/22/02 & CCTV & I & NA & $\begin{array}{l}\text { CCTV was used to check for } \\
\text { leakage at the U2, P4, and P5 } \\
\text { nozzles. No leaks were } \\
\text { observed. }\end{array}$ \\
\hline
\end{tabular}




\begin{tabular}{|c|c|c|c|c|c|c|c|}
\hline \multirow{2}{*}{$\frac{\text { AREA }}{\mathrm{H}}$} & \multirow{2}{*}{$\begin{array}{c}\begin{array}{c}\text { TANK OR } \\
\text { ANCILLARY }\end{array} \\
\text { EVAP-25 }\end{array}$} & \multirow{2}{*}{$\begin{array}{c}\frac{\text { ACCESS OPENING }}{\text { (A OR I) }} \\
\text { NE }\end{array}$} & \multirow{2}{*}{$\frac{\text { DATE }}{04 / 23 / 02}$} & \multicolumn{3}{|c|}{$\begin{array}{l}\text { INSPECTION METHOD } \\
\text { IDENTIFICATION NUMBER }\end{array}$} & \multirow{2}{*}{$\begin{array}{l}\text { REMARKS } \\
\text { CCTV was used to check for } \\
\text { leakage at the U2 and P5 } \\
\text { nozzles. No leaks were } \\
\text { observed. }\end{array}$} \\
\hline & & & & CCTV & l & $811 \mathrm{~J}$ & \\
\hline $\mathrm{H}$ & EVAP-25 & $\mathrm{NE}$ & 04/24/02 & CCTV & l & $811 \mathrm{~J}$ & $\begin{array}{l}\text { CCTV was used to check for } \\
\text { leakage at the U2 and P5 } \\
\text { nozzles. No leaks were } \\
\text { observed. }\end{array}$ \\
\hline $\mathrm{H}$ & EVAP-25 & $\mathrm{NE}$ & 04/25/02 & CCTV & I & $811 \mathrm{~J}$ & $\begin{array}{l}\text { CCTV was used to check for } \\
\text { leakage at the U2 nozzle. No } \\
\text { leaks were observed. }\end{array}$ \\
\hline $\mathrm{H}$ & EVAP-25 & $\mathrm{NE}$ & 04/26/02 & CCTV & I & $811 \mathrm{~J}$ & $\begin{array}{l}\text { CCTV was used to check for } \\
\text { leakage at the U2 and P5 } \\
\text { nozzles. No leaks were } \\
\text { observed. }\end{array}$ \\
\hline $\mathrm{H}$ & EVAP-25 & NE & 04/27/02 & CCTV & l & $811 \mathrm{~J}$ & $\begin{array}{l}\text { CCTV was used to check for } \\
\text { leakage at the U2 and P5 } \\
\text { nozzles. No leaks were } \\
\text { observed. }\end{array}$ \\
\hline $\mathrm{H}$ & EVAP-25 & $\mathrm{NE}$ & 04/28/02 & CCTV & I & $811 \mathrm{~J}$ & $\begin{array}{l}\text { CCTV was used to check for } \\
\text { leakage at the U2 and P5 } \\
\text { nozzles. No leaks were } \\
\text { observed. }\end{array}$ \\
\hline $\mathrm{H}$ & EVAP-25 & $\mathrm{NE}$ & 04/29/02 & CCTV & l & $811 \mathrm{~J}$ & $\begin{array}{l}\text { CCTV was used to check for } \\
\text { leakage at the U2 and P5 } \\
\text { nozzles. No leaks were } \\
\text { observed. }\end{array}$ \\
\hline $\mathrm{H}$ & EVAP-25 & NE & 04/30/02 & CCTV & l & $811 \mathrm{~J}$ & $\begin{array}{l}\text { CCTV was used to check for } \\
\text { leakage at the U2 and P5 } \\
\text { nozzles. No leaks were } \\
\text { observed. }\end{array}$ \\
\hline $\mathrm{H}$ & EVAP-25 & NE & 05/01/02 & CCTV & l & $811 \mathrm{~K}$ & $\begin{array}{l}\text { CCTV was used to document } \\
\text { cell condition during evaporator } \\
\text { operation. No unusual } \\
\text { conditions were observed. }\end{array}$ \\
\hline $\mathrm{H}$ & EVAP-25 & $\mathrm{NE}$ & 05/02/02 & CCTV & l & $811 \mathrm{~K}$ & $\begin{array}{l}\text { CCTV was used to check for } \\
\text { leakage at the U2 nozzle. No } \\
\text { leaks were observed. }\end{array}$ \\
\hline $\mathrm{H}$ & EVAP-25 & $\mathrm{NE}$ & 05/03/02 & CCTV & l & $811 \mathrm{~K}$ & $\begin{array}{l}\text { CCTV was used to check for } \\
\text { leakage at the U2 nozzle. No } \\
\text { leaks were observed. }\end{array}$ \\
\hline $\mathrm{H}$ & EVAP-25 & $\mathrm{NE}$ & 05/04/02 & CCTV & l & $811 \mathrm{~K}$ & $\begin{array}{l}\text { CCTV was used to check for } \\
\text { leakage at the U2 nozzle. No } \\
\text { leaks were observed. }\end{array}$ \\
\hline
\end{tabular}




\begin{tabular}{|c|c|c|c|c|c|c|c|}
\hline \multirow{2}{*}{$\frac{\text { AREA }}{\mathrm{H}}$} & \multirow{2}{*}{$\begin{array}{c}\text { TANK OR } \\
\text { ANCILLARY } \\
\text { EVAP-25 }\end{array}$} & \multirow{2}{*}{$\begin{array}{c}\frac{\text { ACCESS OPENING }}{\text { (A OR I) }} \\
\text { NE }\end{array}$} & \multirow{2}{*}{$\frac{\text { DATE }}{05 / 05 / 02}$} & \multicolumn{3}{|c|}{$\begin{array}{l}\text { INSPECTION METHOD } \\
\text { IDENTIFICATION NUMBER }\end{array}$} & \multirow{2}{*}{$\begin{array}{l}\text { REMARKS } \\
\text { CCTV was used to check for } \\
\text { leakage at the U2 nozzle. No } \\
\text { leaks were observed. }\end{array}$} \\
\hline & & & & CCTV & I & $811 \mathrm{~K}$ & \\
\hline $\mathrm{H}$ & EVAP-25 & NE & 05/07/02 & CCTV & I & $811 \mathrm{~K}$ & $\begin{array}{l}\text { CCTV was used to check for } \\
\text { leakage at the U2 nozzle. No } \\
\text { leaks were observed. }\end{array}$ \\
\hline $\mathrm{H}$ & EVAP-25 & $\mathrm{NE}$ & 05/08/02 & CCTV & I & $811 \mathrm{~K}$ & $\begin{array}{l}\text { CCTV was used to check for } \\
\text { leakage at the U2 nozzle. No } \\
\text { leaks were observed. }\end{array}$ \\
\hline $\mathrm{H}$ & EVAP-25 & $\mathrm{NE}$ & 05/31/02 & CCTV & I & NA & $\begin{array}{l}\text { CCTV was used to check for } \\
\text { leakage at the U2 nozzle. No } \\
\text { leaks were observed. }\end{array}$ \\
\hline $\mathrm{H}$ & EVAP-25 & $\mathrm{NE}$ & 06/01/02 & CCTV & I & 837 & $\begin{array}{l}\text { CCTV was used to check for } \\
\text { leakage at the U2 nozzle. No } \\
\text { leaks were observed. }\end{array}$ \\
\hline $\mathrm{H}$ & EVAP-25 & $\mathrm{NE}$ & 08/01/02 & CCTV & l & 853 & $\begin{array}{l}\text { CCTV was used to inspect the } \\
\text { electrical jumper Y555-100-133 } \\
\text { connected to the west lift jumper } \\
\text { due to a loss of continuity } \\
\text { through the jumper. Inspection } \\
\text { could not determine cause of } \\
\text { failure. }\end{array}$ \\
\hline $\mathrm{H}$ & EVAP-25 & $\mathrm{NE}$ & 08/28/02 & CCTV & l & 853 & $\begin{array}{l}\text { The evaporator cell, jumpers, } \\
\text { and service piping were } \\
\text { inspected. No unusual } \\
\text { conditions were observed. }\end{array}$ \\
\hline $\mathrm{H}$ & EVAP-25 & Open Cell & 03/21/02 & CCTV & I & $811 F \& G$ & $\begin{array}{l}\text { Inspection of the U2 and P5 } \\
\text { nozzle faces revealed some } \\
\text { gasket material and evidence of } \\
\text { some erosion of the metal } \\
\text { surface. }\end{array}$ \\
\hline $\mathrm{H}$ & EVAP-25 & Open Cell & 03/21/02 & CCTV & l & $811 \mathrm{G}$ & $\begin{array}{l}\text { CCTV was used to facilitate the } \\
\text { remote removal and replacement } \\
\text { of jumper } \\
\text { Y555-100-121,122,123\&124. }\end{array}$ \\
\hline $\mathrm{H}$ & EVAP-25 & Open Cell & 03/22/02 & CCTV & I & $811 \mathrm{G}$ & $\begin{array}{l}\text { Inspection verified that the } \\
\text { condenser cell vent line was } \\
\text { unobstructed. }\end{array}$ \\
\hline
\end{tabular}




\begin{tabular}{|c|c|c|c|c|c|c|c|}
\hline \multirow{2}{*}{$\frac{\text { AREA }}{\mathrm{H}}$} & \multirow{2}{*}{$\begin{array}{c}\text { TANK OR } \\
\text { ANCILLARY } \\
\text { EVAP-25 }\end{array}$} & \multirow{2}{*}{$\begin{array}{l}\frac{\text { ACCESS OPENING }}{(\text { A OR I) }} \\
\text { Open Cell }\end{array}$} & \multirow{2}{*}{$\frac{\text { DATE }}{04 / 02 / 02}$} & \multicolumn{3}{|c|}{$\begin{array}{l}\text { INSPECTION METHOD } \\
\text { IDENTIFICATION NUMBER }\end{array}$} & \multirow[b]{2}{*}{$\begin{array}{l}\text { REMARKS } \\
\text { CCTV was used to document the } \\
\text { conditions of the U2 and P5 } \\
\text { nozzles and the connector heads } \\
\text { of the Y-555-100-121,124 } \\
\text { jumper. Gasket material was } \\
\text { observed on all surfaces, and } \\
\text { erosion was observed on the P5 } \\
\text { and U2 nozzle faces with } \\
\text { possible shallow pitting on the } \\
\text { connector head. }\end{array}$} \\
\hline & & & & CCTV & / & 8111 & \\
\hline $\mathrm{H}$ & EVAP-25 & Open Cell & 04/10/02 & CCTV & I & 8111 & $\begin{array}{l}\text { Inspection verified that the } \\
\text { condenser cell vent line was } \\
\text { unobstructed. }\end{array}$ \\
\hline $\mathrm{H}$ & EVAP-25 & $\begin{array}{r}\text { Overhead } \\
\text { Tank \#1 }\end{array}$ & 07/29/02 & CCTV & I & 853 & $\begin{array}{l}\text { CCTV revealed minimal } \\
\text { sediment and mercury. The } \\
\text { level was below the suction line. }\end{array}$ \\
\hline $\mathrm{H}$ & EVAP-25 & $\begin{array}{r}\text { Overhead } \\
\text { Tank \#2 }\end{array}$ & 07/29/02 & CCTV & l & 853 & $\begin{array}{l}\text { CCTV revealed minimal } \\
\text { sediment and mercury. The } \\
\text { level was below the suction line. }\end{array}$ \\
\hline $\mathrm{H}$ & EVAP-25 & $\begin{array}{r}\text { Overhead } \\
\text { tanks }\end{array}$ & 02/11/02 & CCTV & I & $811 A, B$ \& C & $\begin{array}{l}\text { CCTV was used to assist in the } \\
\text { flushing of the overhead tanks. }\end{array}$ \\
\hline $\mathrm{H}$ & EVAP-25 & SE & 03/10/02 & CCTV & / & $811 \mathrm{D}$ & $\begin{array}{l}\text { CCTV was used to monitor } \\
\text { flushing of the cell. }\end{array}$ \\
\hline $\mathrm{H}$ & EVAP-25 & SE & 03/11/02 & CCTV & I & $811 \mathrm{E}$ & $\begin{array}{l}\text { CCTV was used to monitor } \\
\text { flushing of the cell. Evidence of } \\
\text { leakage was observed on valve } \\
1055 \text { at the valve body. }\end{array}$ \\
\hline $\mathrm{H}$ & EVAP-25 & SE & 03/12/02 & CCTV & I & $811 \mathrm{E}$ & $\begin{array}{l}\text { CCTV was used to monitor } \\
\text { flushing of the cell. }\end{array}$ \\
\hline $\mathrm{H}$ & EVAP-25 & SE & 03/13/02 & CCTV & l & $811 \mathrm{E}$ & $\begin{array}{l}\text { CCTV was used to monitor } \\
\text { flushing of the cell. }\end{array}$ \\
\hline $\mathrm{H}$ & EVAP-25 & SE & 03/16/02 & CCTV & / & $811 \mathrm{~F}$ & $\begin{array}{l}\text { CCTV was used to investigate } \\
\text { an increase in the sump level. } \\
\text { No unusual conditions were } \\
\text { observed. }\end{array}$ \\
\hline $\mathrm{H}$ & EVAP-25 & SE & 03/17/02 & CCTV & l & $811 \mathrm{~F}$ & $\begin{array}{l}\text { CCTV was used to monitor } \\
\text { flushing of the cell and } \\
\text { components. }\end{array}$ \\
\hline $\mathrm{H}$ & EVAP-25 & $\mathrm{SE}$ & 03/29/02 & CCTV & I & $811 \mathrm{H}$ & $\begin{array}{l}\text { CCTV was used to leak check } \\
\text { valve } 1055 \text { and the U2 nozzle } \\
\text { during evaporator operation. } \\
\text { Valve } 1055 \text { was leak free; } \\
\text { however, inspection revealed } \\
\text { salt accumulation at the U2 } \\
\text { nozzle. }\end{array}$ \\
\hline
\end{tabular}




\begin{tabular}{|c|c|c|c|c|c|c|c|}
\hline \multirow{2}{*}{$\frac{\text { AREA }}{\mathrm{H}}$} & \multirow{2}{*}{$\begin{array}{c}\text { TANK OR } \\
\text { ANCILLARY } \\
\text { EVAP-25 }\end{array}$} & \multirow{2}{*}{$\begin{array}{c}\frac{\text { ACCESS OPENING }}{\text { (A OR I) }} \\
\text { SE }\end{array}$} & \multirow{2}{*}{$\frac{\text { DATE }}{08 / 29 / 02}$} & \multicolumn{3}{|c|}{$\begin{array}{l}\text { INSPECTION METHOD } \\
\text { IDENTIFICATION NUMBER }\end{array}$} & \multirow{2}{*}{$\begin{array}{l}\text { REMARKS } \\
\text { CCTV was used to leak check } \\
\text { jumpers Y555-134-2 and } \\
\text { Y555-100-131. No leaks were } \\
\text { observed. }\end{array}$} \\
\hline & & & & CCTV & I & 862 & \\
\hline $\mathrm{H}$ & EVAP-25 & SE & 09/12/02 & CCTV & I & 862 & $\begin{array}{l}\text { CCTV was used to monitor } \\
\text { thermal expansion of the } \\
\text { evaporator vessel at the U2 and } \\
\text { P5 nozzles. No unusual } \\
\text { conditions were observed. }\end{array}$ \\
\hline $\mathrm{H}$ & EVAP-25 & SE & 09/12/02 & CCTV & I & 862 & $\begin{array}{l}\text { CCTV was used to monitor } \\
\text { thermal expansion of the } \\
\text { evaporator vessel at the U2 and } \\
\text { P5 nozzles. No unusual } \\
\text { conditions were observed. }\end{array}$ \\
\hline $\mathrm{H}$ & EVAP-25 & SE & 09/13/02 & CCTV & I & 867 & $\begin{array}{l}\text { CCTV was used to monitor } \\
\text { thermal expansion of the } \\
\text { evaporator vessel at the U2 and } \\
\text { P5 nozzles. No unusual } \\
\text { conditions were observed. }\end{array}$ \\
\hline $\mathrm{H}$ & EVAP-25 & SE & 09/14/02 & CCTV & I & 868 & $\begin{array}{l}\text { CCTV was used to monitor } \\
\text { thermal expansion of the } \\
\text { evaporator vessel and the U2 } \\
\text { and P5 nozzles. No unusual } \\
\text { conditions were observed. }\end{array}$ \\
\hline $\mathrm{H}$ & EVAP-25 & SE & 09/15/02 & CCTV & I & 868 & $\begin{array}{l}\text { CCTV was used to monitor } \\
\text { thermal expansion of the } \\
\text { evaporator vessel and the U2 } \\
\text { and P5 nozzles. No unusual } \\
\text { conditions were observed. }\end{array}$ \\
\hline $\mathrm{H}$ & EVAP-25 & SE & 09/15/02 & CCTV & I & 868 & $\begin{array}{l}\text { CCTV was used to monitor the } \\
\text { U2 and P5 nozzles. No unusual } \\
\text { conditions were observed. }\end{array}$ \\
\hline $\mathrm{H}$ & EVAP-25 & SE & 09/26/02 & CCTV & I & 868 & $\begin{array}{l}\text { CCTV was used to monitor the } \\
\text { U2 and P5 nozzles. No unusual } \\
\text { conditions were observed. }\end{array}$ \\
\hline $\mathrm{H}$ & EVAP-25 & SE & $10 / 20 / 02$ & CCTV & I & 868 & $\begin{array}{l}\text { CCTV was used to monitor the } \\
\text { U2 and P5 nozzles. No unusual } \\
\text { conditions were observed. }\end{array}$ \\
\hline $\mathrm{H}$ & EVAP-25 & SE & $10 / 21 / 02$ & CCTV & I & 868 & $\begin{array}{l}\text { CCTV was used to monitor the } \\
\text { U2 and P5 nozzles. No unusual } \\
\text { conditions were observed. }\end{array}$ \\
\hline $\mathrm{H}$ & EVAP-25 & SE & $10 / 22 / 02$ & CCTV & I & 868 & $\begin{array}{l}\text { CCTV was used to monitor the } \\
\text { U2 and P5 nozzles. No unusual } \\
\text { conditions were observed. }\end{array}$ \\
\hline
\end{tabular}




\begin{tabular}{|c|c|c|c|c|c|c|c|}
\hline \multirow{2}{*}{$\frac{\text { AREA }}{\mathrm{H}}$} & \multirow{2}{*}{$\begin{array}{c}\text { TANK OR } \\
\text { ANCILLARY } \\
\text { EVAP-25 }\end{array}$} & \multirow{2}{*}{$\frac{\frac{\text { ACCESS OPENING }}{\text { (A OR I) }}}{\text { SE }}$} & \multirow{2}{*}{$\frac{\text { DATE }}{10 / 24 / 02}$} & \multicolumn{3}{|c|}{$\begin{array}{l}\text { INSPECTION METHOD } \\
\text { IDENTIFICATION NUMBER }\end{array}$} & \multirow{2}{*}{$\begin{array}{l}\text { REMARKS } \\
\text { CCTV was used to monitor the } \\
\text { U2 and P5 nozzles. No unusual } \\
\text { conditions were observed. }\end{array}$} \\
\hline & & & & CCTV & I & 868 & \\
\hline $\mathrm{H}$ & EVAP-25 & SE & $10 / 27 / 02$ & CCTV & / & 868 & $\begin{array}{l}\text { CCTV was used to monitor the } \\
\text { U2 and P5 nozzles. No unusual } \\
\text { conditions were observed. }\end{array}$ \\
\hline $\mathrm{H}$ & EVAP-25 & SE & $10 / 30 / 02$ & CCTV & I & 868 & $\begin{array}{l}\text { CCTV was used to monitor the } \\
\text { U2 and P5 nozzles. No unusual } \\
\text { conditions were observed. }\end{array}$ \\
\hline $\mathrm{H}$ & EVAP-25 & SE & $10 / 31 / 02$ & CCTV & I & 868 & $\begin{array}{l}\text { CCTV was used to monitor the } \\
\text { U2 and P5 nozzles. No unusual } \\
\text { conditions were observed. }\end{array}$ \\
\hline $\mathrm{H}$ & EVAP-25 & SE & $11 / 01 / 02$ & CCTV & I & 868 & $\begin{array}{l}\text { CCTV was used to monitor the } \\
\text { U2 and P5 nozzles. No unusual } \\
\text { conditions were observed. }\end{array}$ \\
\hline $\mathrm{H}$ & EVAP-25 & SE & $11 / 04 / 02$ & CCTV & I & 868 & $\begin{array}{l}\text { CCTV was used to monitor the } \\
\text { U2 and P5 nozzles. No unusual } \\
\text { conditions were observed. }\end{array}$ \\
\hline $\mathrm{H}$ & EVAP-25 & SE & $11 / 24 / 02$ & CCTV & I & 868 & $\begin{array}{l}\text { CCTV was used to monitor the } \\
\text { U2 nozzle. No unusual } \\
\text { conditions were observed. }\end{array}$ \\
\hline $\mathrm{H}$ & EVAP-25 & SE & $11 / 27 / 02$ & CCTV & I & 868 & $\begin{array}{l}\text { CCTV was used to monitor the } \\
\text { U2 nozzle. No unusual } \\
\text { conditions were observed. }\end{array}$ \\
\hline $\mathrm{H}$ & EVAP-25 & SE & $12 / 02 / 02$ & CCTV & I & 868 & $\begin{array}{l}\text { CCTV was used to monitor the } \\
\text { U2 nozzle. No unusual } \\
\text { conditions were observed. }\end{array}$ \\
\hline $\mathrm{H}$ & EVAP-25 & SE & $12 / 03 / 02$ & CCTV & I & 868 & $\begin{array}{l}\text { CCTV was used to monitor the } \\
\text { U2 nozzle. No unusual } \\
\text { conditions were observed. }\end{array}$ \\
\hline $\mathrm{H}$ & EVAP-25 & SE & $12 / 09 / 02$ & CCTV & I & 868 & $\begin{array}{l}\text { CCTV was used to monitor the } \\
\text { U2 nozzle. No unusual } \\
\text { conditions were observed. }\end{array}$ \\
\hline $\mathrm{H}$ & EVAP-25 & SE & $12 / 12 / 02$ & CCTV & I & 868 & $\begin{array}{l}\text { CCTV was used to monitor the } \\
\text { U2 nozzle. No unusual } \\
\text { conditions were observed. }\end{array}$ \\
\hline $\mathrm{H}$ & EVAP-25 & SE & $12 / 12 / 02$ & CCTV & I & 868 & $\begin{array}{l}\text { CCTV was used to monitor the } \\
\text { U2 nozzle. No unusual } \\
\text { conditions were observed. }\end{array}$ \\
\hline $\mathrm{H}$ & EVAP-25 & SE & $12 / 15 / 02$ & CCTV & I & 868 & $\begin{array}{l}\text { CCTV was used to monitor the } \\
\text { U2 nozzle. No unusual } \\
\text { conditions were observed. }\end{array}$ \\
\hline
\end{tabular}




\begin{tabular}{|c|c|c|c|c|c|c|c|}
\hline \multirow{2}{*}{$\frac{\text { AREA }}{H}$} & \multirow{2}{*}{$\begin{array}{c}\begin{array}{c}\text { TANK OR } \\
\text { ANCILLARY }\end{array} \\
\text { EVAP-25 }\end{array}$} & \multirow{2}{*}{$\begin{array}{c}\frac{\text { ACCESS OPENING }}{\text { (A OR I) }} \\
\text { SE }\end{array}$} & \multirow{2}{*}{$\frac{\text { DATE }}{12 / 16 / 02}$} & \multicolumn{3}{|c|}{$\begin{array}{l}\text { INSPECTION METHOD } \\
\text { IDENTIFICATION NUMBER }\end{array}$} & \multirow{2}{*}{$\begin{array}{l}\quad \text { REMARKS } \\
\text { CCTV was used to monitor the } \\
\text { U2 nozzle. No unusual } \\
\text { conditions were observed. }\end{array}$} \\
\hline & & & & CCTV & I & 868 & \\
\hline $\mathrm{H}$ & EVAP-25 & SE & $12 / 24 / 02$ & CCTV & I & 903 & $\begin{array}{l}\text { CCTV was used to monitor the } \\
\text { U2 nozzle. No unusual } \\
\text { conditions were observed. }\end{array}$ \\
\hline $\mathrm{H}$ & EVAP-25 & SE & $12 / 25 / 02$ & CCTV & I & 903 & $\begin{array}{l}\text { CCTV was used to monitor the } \\
\text { U2 nozzle. No unusual } \\
\text { conditions were observed. }\end{array}$ \\
\hline $\mathrm{H}$ & EVAP-25 & SE & $12 / 26 / 02$ & CCTV & I & 903 & $\begin{array}{l}\text { CCTV was used to monitor the } \\
\text { U2 nozzle. No unusual } \\
\text { conditions were observed. }\end{array}$ \\
\hline $\mathrm{H}$ & EVAP-25 & $\begin{array}{r}\text { Separator } \\
\text { Pot }\end{array}$ & 03/16/02 & CCTV & I & $811 \mathrm{~F}$ & $\begin{array}{l}\text { CCTV was used to inspect the } \\
\text { separator pot. Liquid was } \\
\text { observed in the pot just below } \\
\text { the inlet side. Liquid was } \\
\text { observed in the catheter line, } \\
\text { and solids were observed at the } \\
\text { bottom of the separator pot } \\
\text { plugging the outlet. }\end{array}$ \\
\hline $\mathrm{H}$ & EVAP-25 & $\begin{array}{r}\text { Separator } \\
\text { Pot }\end{array}$ & 03/17/02 & CCTV & I & $811 \mathrm{~F}$ & $\begin{array}{l}\text { CCTV was used to inspect the } \\
\text { separator pot after pressure } \\
\text { washing. The pot was free of } \\
\text { solids, and no liquid or blockage } \\
\text { was observed. }\end{array}$ \\
\hline $\mathrm{H}$ & EVAP-25 & SW & 01/04/02 & CCTV & I & $759 B$ & $\begin{array}{l}\text { CCTV was used to perform leak } \\
\text { check of the S6 flange. No } \\
\text { evidence of leakage was found } \\
\text { around the flange or on the } \\
\text { evaporator pot. Some damp } \\
\text { areas were observed on the top } \\
\text { surface of the S6 flange. No } \\
\text { liquid or steam was observed } \\
\text { inside the evaporator cell. }\end{array}$ \\
\hline $\mathrm{H}$ & EVAP-25 & SW & 08/28/02 & CCTV & / & 853 & $\begin{array}{l}\text { The evaporator cell, jumpers, } \\
\text { and service piping were } \\
\text { inspected. No unusual } \\
\text { conditions were observed. }\end{array}$ \\
\hline $\mathrm{H}$ & EVAP-25 & SW & 09/09/02 & CCTV & 1 & 862 & $\begin{array}{l}\text { CCTV was used to leak check } \\
\text { the S6 flange. No leaks were } \\
\text { observed. }\end{array}$ \\
\hline
\end{tabular}




\begin{tabular}{|c|c|c|c|c|c|c|}
\hline \multirow{2}{*}{$\frac{\text { AREA }}{\mathrm{H}}$} & \multirow{2}{*}{$\begin{array}{c}\begin{array}{c}\text { TANK OR } \\
\text { ANCILLARY }\end{array} \\
\text { EVAP-25 }\end{array}$} & $\frac{\text { ACCESS OPENING }}{(\text { A OR I) }}$ & \multirow{2}{*}{$\frac{\text { DATE }}{06 / 04 / 02}$} & \multicolumn{2}{|c|}{$\begin{array}{l}\text { INSPECTION METHOD } \\
\text { IDENTIFICATION NUMBER }\end{array}$} & \multirow[b]{2}{*}{$\begin{array}{l}\text { REMARKS } \\
\text { Inspection of the pot showed all } \\
\text { areas to be free of deposits. A } \\
\text { thin scale was observed on the } \\
\text { tube bundle and areas of the pot } \\
\text { wall. Approximately } 6 \text { to } 7 \text { feet } \\
\text { of liquid remained in the pot. } \\
\text { Loose solids were observed on } \\
\text { the top of the warming coil and } \\
\text { support collar. Approximately } 4 \\
\text { inches of loose solids had } \\
\text { settled at the bottom of the cone. } \\
\text { The end of the lift line was not } \\
\text { visible. Observations in this } \\
\text { inspection were consistent with } \\
\text { normal evaporator operation. }\end{array}$} \\
\hline & & S-6 (I) & & CCTV & 837 & \\
\hline
\end{tabular}

$\mathrm{F}$

$\mathrm{F}$

$\mathrm{F}$

F

PP-02

PP-05

PP-05

LDB-05

05/02/02

CCT

CCTV /

841

$\mathrm{H}$

IAL

High Point

Sump

05/19/02

CCTV

I

809G

Sump

$11 / 12 / 02$

CCTV

I

866

$10 / 30 / 02$

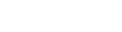

$\mathrm{H}$

PP-05

LDB-05

10/30/02

CCTV /

841
CCTV revealed that the conductivity probes were properly deployed and that the drain plugs were installed.

CCTV was used to leak check instrument jumper 18(FPP2)6. No leaks were observed, and the pump tank passive vent and the pump pit overflow pipe were free of obstructions.

CCTV was used to verify proper agitator pump rotation. The pump was rotating in the clockwise direction.

CCTV was used to locate the source of water inleakage. There was no evidence of inleakage, and no water was observed entering the sump. However, the inspection was not adequate enough to provide coverage for the entire pump pit.

The conductivity probe was deployed within the setpoint limits.

The conductivity probe was deployed within the setpoint limits.

The conductivity probe was deployed within the setpoint limits. 


\begin{tabular}{|c|c|c|c|c|c|c|c|}
\hline \multirow{2}{*}{$\frac{\text { AREA }}{\mathrm{H}}$} & \multirow{2}{*}{$\begin{array}{c}\text { TANK OR } \\
\text { ANCILLARY } \\
\text { PP-06 }\end{array}$} & \multirow{2}{*}{$\begin{array}{l}\frac{\text { ACCESS OPENING }}{(\text { A OR I) }} \\
\text { LDB-07 }\end{array}$} & \multirow{2}{*}{$\frac{\text { DATE }}{05 / 01 / 02}$} & \multicolumn{3}{|c|}{$\frac{\text { INSPECTION METHOD }}{\text { IDENTIFICATION NUMBER }}$} & \multirow{2}{*}{$\begin{array}{l}\text { REMARKS } \\
\text { The conductivity probe was } \\
\text { deployed within the setpoint } \\
\text { limits. }\end{array}$} \\
\hline & & & & CCTV & I & $807 A$ & \\
\hline $\mathrm{H}$ & PP-06 & SW & 06/28/02 & CCTV & I & 845 & $\begin{array}{l}\text { Inspection verified that jumper } \\
1 \text { (HPP6)15A was leak free. }\end{array}$ \\
\hline $\mathrm{H}$ & PP-07 & NE & $11 / 15 / 02$ & CCTV & I & 866 & $\begin{array}{l}\text { CCTV was used to document } \\
\text { instrument air line configuration } \\
\text { for jumper 22(HPP7)23x,23y. }\end{array}$ \\
\hline$F$ & SWS & $4 \mathrm{~F}-04$ & $10 / 05 / 02$ & CCTV & I & 866 & $\begin{array}{l}\text { Inspection documented the } \\
\text { conditions of the south and east } \\
\text { gates. The east gate was } \\
\text { closed. No obstructions were } \\
\text { observed in the storm drain. No } \\
\text { significant erosion was observed } \\
\text { at the south gate, and conditions } \\
\text { were consistent with normal } \\
\text { operations. }\end{array}$ \\
\hline $\mathrm{F}$ & SWS & $4 \mathrm{~F}-04$ & $10 / 23 / 02$ & CCTV & I & 866 & $\begin{array}{l}\text { Inspection documented the } \\
\text { conditions of the south and east } \\
\text { gates. No obstructions were } \\
\text { observed in the storm drain. No } \\
\text { significant erosion was observed } \\
\text { at the south or east gates, and } \\
\text { conditions were consistent with } \\
\text { normal operations. }\end{array}$ \\
\hline $\mathrm{F}$ & SWS & $4 \mathrm{~F}-04$ & $10 / 23 / 02$ & CCTV & I & 866 & $\begin{array}{l}\text { Inspection documented the } \\
\text { condition of the south and east } \\
\text { gates. No debris was observed. } \\
\text { The conditions were consistent } \\
\text { with normal operation. }\end{array}$ \\
\hline
\end{tabular}




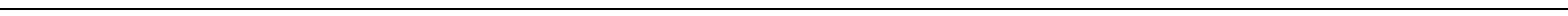

